\title{
Multidimensional Laplace Transforms over Quaternions, Octonions and Cayley-Dickson Algebras, Their Applications to PDE
}

\author{
Sergey Victor Ludkovsky \\ Department of Applied Mathematics, Moscow State Technical University, Moscow, Russia \\ Email: sludkowski@mail.ru
}

Received July 8, 2011; revised November 10, 2011; accepted November 20, 2011

\begin{abstract}
Multidimensional noncommutative Laplace transforms over octonions are studied. Theorems about direct and inverse transforms and other properties of the Laplace transforms over the Cayley-Dickson algebras are proved. Applications to partial differential equations including that of elliptic, parabolic and hyperbolic type are investigated. Moreover, partial differential equations of higher order with real and complex coefficients and with variable coefficients with or without boundary conditions are considered.
\end{abstract}

Keywords: Laplace Transform; Quaternion Skew Field; Octonion Algebra; Cayley-Dickson Algebra; Partial Differential Equation; Non-Commutative Integration

\section{Introduction}

The Laplace transform over the complex field is already classical and plays very important role in mathematics including complex analysis and differential equations [13]. The classical Laplace transform is used frequently for ordinary differential equations and also for partial differential equations sufficiently simple to be resolved, for example, of two variables. But it meets substantial difficulties or does not work for general partial differential equations even with constant coefficients especially for that of hyperbolic type.

To overcome these drawbacks of the classical Laplace transform in the present paper more general noncommutative multiparameter transforms over Cayley-Dickson algebras are investigated. In the preceding paper a noncommutative analog of the classical Laplace transform over the Cayley-Dickson algebras was defined and investigated [4]. This paper is devoted to its generalizations for several real parameters and also variables in the Cayley-Dickson algebras. For this the preceding results of the author on holomorphic, that is (super) differentiable functions, and meromorphic functions of the Cayley-Dickson numbers are used $[5,6]$. The super-differentiability of functions of Cayley-Dickson variables is stronger than the Fréchet's differentiability. In those works also a noncommutative line integration was investigated.

We remind that quaternions and operations over them had been first defined and investigated by W. R. Hamilton in 1843 [7]. Several years later on Cayley and
Dickson had introduced generalizations of quaternions known now as the Cayley-Dickson algebras [8-11]. These algebras, especially quaternions and octonions, have found applications in physics. They were used by Maxwell, Yang and Mills while derivation of their equations, which they then have rewritten in the real form because of the insufficient development of mathematical analysis over such algebras in their time [12-14]. This is important, because noncommutative gauge fields are widely used in theoretical physics [15].

Each Cayley-Dickson algebra $A_{r}$ over the real field $R$ has $2^{r}$ generators $\left\{i_{0}, i_{1}, \cdots, i_{2^{r}-1}\right\}$ such that $i_{0}=1$, $i_{j}^{2}=-1$ for each $j=1,2, \cdots, 2^{r}-1, i_{j} i_{k}=-i_{k} i_{j}$ for every $1 \leq k \neq j \leq 2^{r}-1$, where $r \geq 1$. The algebra $A_{r+1}$ is formed from the preceding algebra $A_{r}$ with the help of the so-called doubling procedure by generator $i_{2^{r}}$. In particular, $A_{1}=C$ coincides with the field of complex numbers, $A_{2}=H$ is the skew field of quaternions, $A_{3}$ is the algebra of octonions, $A_{4}$ is the algebra of sedenions. This means that a sequence of embeddings $\cdots \longmapsto A_{r} \longmapsto A_{r+1} \longmapsto \cdots$ exists.

Generators of the Cayley-Dickson algebras have a natural physical meaning as generating operators of fermions. The skew field of quaternions is associative, and the algebra of octonions is alternative. The CayleyDickson algebra $A_{r}$ is power associative, that is, $z^{n+m}=z^{n} z^{m}$ for each $n, m \in N$ and $z \in A_{r}$. It is nonassociative and non-alternative for each $r \geq 4$. A 
conjugation $z^{*}=\tilde{z}$ of Cayley-Dickson numbers $z \in A_{r}$ is associated with the norm $|z|^{2}=z z^{*}=z^{*} z$. The octonion algebra has the multiplicative norm and is the division algebra. Cayley-Dickson algebras $A_{r}$ with $r \geq 4$ are not division algebras and have not multiplicative norms. The conjugate of any Cayley-Dickson number $z$ is given by the formula:

(M1) $z^{*}:=\xi^{*}-\eta l$.

The multiplication in $A_{r+1}$ is defined by the following equation:

(M2) $(\xi+\eta l)(\gamma+\delta l)=(\xi \gamma-\tilde{\delta} \eta)+(\delta \xi+\eta \tilde{\gamma}) l$

for each $\xi, \eta, \gamma, \delta \in A_{r}, z:=\xi+\eta l \in A_{r+1}$, $\zeta:=\gamma+\delta l \in A_{r+1}$.

At the beginning of this article a multiparameter noncommutative transform is defined. Then new types of the direct and inverse noncommutative multiparameter transforms over the general Cayley-Dickson algebras are investigated, particularly, also over the quaternion skew field and the algebra of octonions. The transforms are considered in $A_{r}$ spherical and $A_{r}$ Cartesian coordinates. At the same time specific features of the noncommutative multiparameter transforms are elucidated, for example, related with the fact that in the Cayley-Dickson algebra $A_{r}$ there are $2^{r}-1$ imaginary generators $\left\{i_{1}, \cdots, i_{2^{r}-1}\right\}$ apart from one in the field of complex numbers such that the imaginary space in $A_{r}$ has the dimension $2^{r}-1$. Theorems about properties of images and originals in conjunction with the operations of linear combinations, differentiation, integration, shift and homothety are proved. An extension of the noncommutative multiparameter transforms for generalized functions is given. Formulas for noncommutative transforms of products and convolutions of functions are deduced.

Thus this solves the problem of non-commutative mathematical analysis to develop the multiparameter Laplace transform over the Cayley-Dickson algebras. Moreover, an application of the noncommutative integral transforms for solutions of partial differential equations is described. It can serve as an effective means (tool) to solve partial differential equations with real or complex coefficients with or without boundary conditions and their systems of different types (see also [16]). An algorithm is described which permits to write fundamental solutions and functions of Green's type. A moving boundary problem and partial differential equations with discontinuous coefficients are also studied with the use of the noncommutative transform.

Frequently, references within the same subsection are given without number of the subsection, apart from references when subsection are different.

All results of this paper are obtained for the first time.

\section{Multidimensional Noncommutative Integral Transforms}

\subsection{Definitions Transforms in $A_{r}$ Cartesian Coordinates}

Denote by $A$ the Cayley-Dickson algebra, $0 \leq r$, which may be, in particular, $\mathrm{H}=\mathrm{A}_{2}$ the quaternion skew field or $O=A_{3}$ the octonion algebra. For unification of the notation we put $A_{0}=R, A_{1}=C$. A function

$f: R^{n} \rightarrow A_{r}$ we call a function-original, where $2 \leq r$, $n \in N$, if it fulfills the following conditions (1-5).

1) The function $f(t)$ is almost everywhere continuous on $R^{n}$ relative to the Lebesgue measure $\lambda_{n}$ on $R^{n}$.

2) On each finite interval in $R$ each function $g_{j}\left(t_{j}\right)=f\left(t_{1}, \cdots, t_{n}\right)$ by $t_{j}$ with marked all other variables may have only a finite number of points of discontinuity of the first kind, where $t=\left(t_{1}, \cdots, t_{n}\right) \in R^{n}$, $t_{j} \in R, \quad j=1, \cdots, n$. Recall that a point $u_{0} \in R$ is called a point of discontinuity of the first type, if there exist finite left and right limits

$$
\begin{aligned}
& \lim _{u \rightarrow u_{0}, u<u_{0}} g(u)=: g\left(u_{0}-0\right) \in A_{r} \text { and } \\
& \lim _{u \rightarrow u_{0}, u>u_{0}} g(u)=: g\left(u_{0}+0\right) \in A_{r} .
\end{aligned}
$$

3) Every partial function $g_{j}\left(t_{j}\right)=f\left(t_{1}, \cdots, t_{n}\right)$ satisfies the Hölder condition: $\left|g_{j}\left(t_{j}+h_{j}\right)-g_{j}\left(t_{j}\right)\right| \leq A_{j}\left|h_{j}\right|^{\alpha_{j}}$ for each $\left|h_{j}\right|<\delta$, where $0<\alpha_{j} \leq 1, A_{j}=$ const $>0, \delta_{j}>0$ are constants for a given $t=\left(t_{1}, \cdots, t_{n}\right) \in R^{n}, \quad j=1, \cdots, n$, everywhere on $R^{n}$ may be besides points of discontinuity of the first type.

4) The function $f(t)$ increases not faster, than the exponential function, that is there exist constants

$C_{v}=$ const $>0, \quad v=\left(v_{1}, \cdots, v_{n}\right), \quad a_{-1}, a_{1} \in R$, where $v_{j} \in\{-1,1\}$ for every $j=1, \cdots, n$, such that $|f(t)|<C_{v} \exp \left(\left(q_{v}, t\right)\right)$ for each $t \in R^{n}$ with $t_{j} v_{j} \geq 0$ for each $j=1, \cdots, n, q_{v}=\left(v_{1} a_{v_{1}}, \cdots, v_{n} a_{v_{n}}\right)$; where

5) $(x, y):=\sum_{j=1}^{n} x_{j} y_{j}$ denotes the standard scalar product in $R^{n}$.

Certainly for a bounded original $f$ it is possible to take $a_{-1}=a_{1}=0$.

Each Cayley-Dickson number $p \in A_{r}$ we write in the form

6) $p=\sum_{j=0}^{2^{r}-1} p_{j} i_{j}$, where $\left\{i_{0}, i_{1}, \cdots, i_{2^{r}-1}\right\}$ is the standard basis of generators of $A_{r}$ so that $i_{0}=1, i_{j}^{2}=-1$ and $i_{0} i_{j}=i_{j}=i_{j} i_{0}$ for each $j>0, i_{j} i_{k}=-i_{k} i_{j}$ for each $j>0$ and $k>0$ with $k \neq j, p_{j} \in R$ for each $j$. If there exists an integral

7) $F^{n}(p):=F^{n}(p ; \zeta):=\int_{R^{n}} f(t) e^{-\langle p, t)-\zeta} \mathrm{d} t$, then $F^{n}(p)$ is called the noncommutative multiparameter (Laplace) transform at a point $p \in A_{r}$ of the function-original $f(t)$, where 
$\zeta-\zeta_{0}=\zeta_{1} i_{1}+\cdots+\zeta_{2^{r}-1} i_{2^{r}-1} \in A_{r}$ is the parameter of an initial phase, $\zeta_{j} \in R$ for each $j=0,1, \cdots, 2^{r}-1$, $\zeta \in A_{r}, \quad n=2^{r}-1, \mathrm{~d} t=\lambda_{n}(\mathrm{~d} t)$,

8) $\langle p, t)=p_{0}\left(t_{1}+\cdots+t_{2^{r}-1}\right)+\sum_{j=1}^{2^{r}-1} p_{j} t_{j} i_{j}$,

we also put

8.1) $u(p, t ; \zeta)=\langle p, t)+\zeta$.

For vectors $v, w \in R^{n}$ we shall consider a partial ordering
9) $v \prec w$ if and only if $v_{j} \leq w_{j}$ for each $j=1, \cdots, n$ and a $k$ exists so that $v_{k}<w_{k}$, $1 \leq k \leq n$.

\subsection{Transforms in $A_{r}$ Spherical Coordinates}

Now we consider also the non-linear function $u=u(p, t ; \zeta)$ taking into account non commutativity of the Cayley-Dickson algebra $A_{r}$. Put

1) $u(p, t):=u(p, t ; \zeta):=p_{0} s_{1}+M(p, t)+\zeta_{0}$, where

2) $M(p, t)=M(p, t ; \zeta)=\left(p_{1} s_{1}+\zeta_{1}\right)\left[i_{1} \cos \left(p_{2} s_{2}+\zeta_{2}\right)+i_{2} \sin \left(p_{2} s_{2}+\zeta_{2}\right) \cos \left(p_{3} s_{3}+\zeta_{3}\right)+\cdots+i_{2^{r}-2} \sin \left(p_{2} s_{2}+\zeta_{2}\right) \cdots\right.$

$$
\left.\sin \left(p_{2^{r}-2} S_{2^{r}-2}+\zeta_{2^{r}-2}\right) \cos \left(p_{2^{r}-1} S_{2^{r}-1}+\zeta_{2^{r}-1}\right)+i_{2^{r}-1} \sin \left(p_{2} S_{2}+\zeta_{2}\right) \cdots \sin \left(p_{2^{r}-2} S_{2^{r}-2}+\zeta_{2^{r}-2}\right) \sin \left(p_{2^{r}-1} S_{2^{r}-1}+\zeta_{2^{r}-1}\right)\right]
$$

for the general Cayley-Dickson algebra with $2 \leq r<\infty$.

2.1) $s_{j}:=s_{j}(n ; t):=t_{j}+\cdots+t_{n}$ for each $j=1, \cdots, n$, $n=2^{r}-1$, so that $s_{1}=t_{1}+\cdots+t_{n}, s_{n}=t_{n}$. More generally, let

3) $u(p, t)=u(p, t ; \zeta)=p_{0} s_{1}+w(p, t)+\zeta_{0}$, where $w(p, t)$ is a locally analytic function, $\operatorname{Re}(w(p, t))=0$ for each $p \in A_{r}$ and $t \in R^{2^{r}-1}, \operatorname{Re}(z):=(z+\tilde{z}) / 2$, $\tilde{z}=z^{*}$ denotes the conjugated number for $z \in A_{r}$. Then the more general non-commutative multiparameter transform over $A_{r}$ is defined by the formula:

4) $F_{u}^{n}(p ; \zeta):=\int_{R^{n}} f(t) \exp (-u(p, t ; \zeta)) \mathrm{d} t$

for each Cayley-Dickson numbers $p \in A_{r}$ whenever this integral exists as the principal value of either Riemann or Lebesgue integral, $n=2^{r}-1$. This non-commutative multiparameter transform is in $A_{r}$ spherical coordinates, when $u(p, t ; \zeta)$ is given by Formulas $(1,2)$.

At the same time the components $p_{j}$ of the number $p$ and $\zeta_{j}$ for $\zeta$ in $u(p, t ; \zeta)$ we write in the $p$ and $\zeta$-representations respectively such that

5) $h_{j}=\left(-h i_{j}+i_{j}\left(2^{r}-2\right)^{-1}\left\{-h+\sum_{k=1}^{2^{r}-1} i_{k}\left(h i_{k}^{*}\right)\right\}\right) / 2$ for each $j=1,2, \cdots, 2^{r}-1$,
6) $h_{0}=\left(h+\left(2^{r}-2\right)^{-1}\left\{-h+\sum_{k=1}^{2^{r}-1} i_{k}\left(h i_{k}^{*}\right)\right\}\right) / 2$,

where $2 \leq r \in N, h=h_{0} i_{0}+\cdots+h_{2^{r}-12^{r}-1} \in A_{r}, \quad h_{j} \in R$ for each $j, i_{k}^{*}=\tilde{i}_{k}=-i_{k}$ for each ${ }^{2^{r}}{ }^{-1}>^{2}{ }^{1}, i_{0}=1$, $h \in A_{r}$. Denote $F_{u}^{n}(p ; \zeta)$ in more details by $F^{n}(f, u ; p ; \zeta)$.

Henceforth, the functions $u(p, t ; \zeta)$ given by $1(8,8.1)$ or $(1,2,2.1)$ are used, if another form (3) is not specified. If for $u(p, t ; \zeta)$ concrete formulas are not mentioned, it will be undermined, that the function $u(p, t ; \zeta)$ is given in $A_{r}$ spherical coordinates by Expressions 1,2,2.1). If in Formulas 1(7) or (4) the integral is not by all, but only by $t_{j(1)}, \cdots, t_{j(k)}$ variables, where $1 \leq k<n$, $1 \leq j(1)<\cdots<j(k) \leq n$, then we denote a noncommutative transform by $F_{u}^{k ; t}{ }_{j(1)}, \cdots, t_{j(k)}(p ; \zeta)$ or $F^{k ; t_{j(1)}, \cdots, t_{j(k)}}(f, u ; p ; \zeta)$. If $j(1)=1, \cdots, j(k)=k$, then we denote it shortly by $F_{u}^{k}(p ; \zeta)$ or $F^{k}(f, u ; p ; \zeta)$. Henceforth, we take $\zeta_{m}=0$ and $t_{m}=0$ and $p_{m}=0$ for each $1 \leq m \notin\{j(1), \cdots, j(k)\}$ if something other is not specified.

\subsection{Remark}

The spherical $A_{r}$ coordinates appear naturally from the following consideration of iterated exponents:

$$
\text { 1) } \begin{aligned}
& \exp \left(i _ { 1 } ( p _ { 1 } s _ { 1 } + \zeta _ { 1 } ) \left(\exp \left(-i_{3}\left(p_{2} s_{2}+\zeta_{2}\right)\left(\exp \left(-i_{1}\left(p_{3} s_{3}+\zeta_{3}\right)\right)\right)\right)\right.\right. \\
& =\exp \left(\left(p_{1} s_{1}+\zeta_{1}\right)\left(i_{1} \cos \left(p_{2} s_{2}+\zeta_{2}\right)+i_{2} \sin \left(p_{2} s_{2}+\zeta_{2}\right) \cos \left(p_{3} s_{3}+\zeta_{3}\right)+i_{3} \sin \left(p_{2} s_{2}+\zeta_{2}\right) \sin \left(p_{3} s_{3}+\zeta_{3}\right)\right)\right)
\end{aligned}
$$

Consider $i_{2}$ the generator of the doubling procedure of the Cayley-Dickson algebra $A_{r+1}$ from the CayleyDickson algebra $A_{r}$, such that $i_{j} i_{2 r}=i_{2^{r}+j}$ for each $j=0, \cdots, 2^{r}-1$. We denote now the function

$M(p, t ; \zeta)$ from Definition 2 over $A_{r}$ in more details by ${ }_{r} M$. Then by induction we write:

2) $\exp \left({ }_{r+1} M(p, t ; \zeta)\right)=\exp \left\{{ }_{r} M\left(\left(i_{1} p_{1}+\cdots+i_{2^{r}-1} p_{2^{r}-1}\right),\left(t_{1}, \cdots, t_{2^{r}-2},\left(t_{2^{r}-1}+s_{2^{r}}\right)\right) ;\left(i_{1} \zeta_{1}+\cdots+i_{2^{r}-1} \zeta_{2^{r}-1}\right)\right.\right.$

$$
\exp \left(-i_{2^{r}+1}\left(p_{2^{r}{ }^{r}} s+\zeta_{2^{r}}\right) \exp \left(-{ }_{r} M\left(\left(i_{1} p_{2^{r}+1}+\cdots+i_{2^{r}-1} p_{2^{r+1}-1}\right),\left(t_{2^{r}+1}, \cdots, t_{2^{r+1}-1}\right) ;\left(i_{1} \zeta_{2^{r}+1}+\cdots+i_{2^{r}-1} \zeta_{2^{r+1}-1}\right)\right)\right)\right\}
$$


where $t=\left(t_{1}, \cdots, t_{n}\right), n=n(r+1)=2^{r+1}-1$, $s_{j}=s_{j}(n(r+1) ; t)$ for each $j=1, \cdots, n(r+1)$, since

$$
\begin{aligned}
s_{m}(n(r+1) ; t) & =t_{m}+\cdots+t_{n(r+1)} \\
& =s_{m}(n(r) ; t)+s_{2^{r}}(n(r+1) ; t)
\end{aligned}
$$

for each $m=1, \cdots, 2^{r}-1$.

An image function can be written in the form

3) $F_{u}^{n}(p ; \zeta):=\sum_{j=0}^{2^{r}-1} i_{j} F_{u, j}^{n}(p ; \zeta)$,

where a function $f$ is decomposed in the form

3.1) $f(t)=\sum_{j=0}^{2^{r}-1} i_{j} f_{j}(t)$,

$f_{j}: R^{n} \rightarrow R$ for each $j=0,1, \cdots, 2^{r}-1, \quad F_{u, j}^{n}(p ; \zeta)$

de-notes the image of the function-original $f_{j}$.

If an automorphism of the Cayley-Dickson algebra $A_{r}$ is taken and instead of the standard generators $\left\{i_{0}, \cdots, i_{2^{r}-1}\right\}$ new generators $\left\{N_{0}, \cdots, N_{2^{r}-1}\right\}$ are used, this provides also $M(p, t ; \zeta)=M_{N}(p, t ; \zeta)$ relative to new basic generators, where $2 \leq r \in N$. In this more general case we denote by ${ }_{N} F_{u}^{n}(p ; \zeta)$ an image for an original $f(t)$, or in more details we denote it by

${ }_{N} F^{n}(f, u ; p ; \zeta)$.

Formulas 1(7) and 2(4) define the right multiparameter transform. Symmetrically is defined a left multiparameter transform. They are related by conjugation and up to a sign of basic generators. For real valued originals they certainly coincide. Henceforward, only the right multiparameter transform is investigated.
Particularly, if $p=\left(p_{0}, p_{1}, 0, \cdots, 0\right)$ and $t=\left(t_{1}, 0, \cdots, 0\right)$, then the multiparameter non-commutative Laplace transforms 1(7) and 2(4) reduce to the complex case, with parameters $a_{1}, a_{-1}$. Thus, the given above definitions over quaternions, octonions and general Cayley-Dickson algebras are justified.

\subsection{Theorem}

If an original $f(t)$ satisfies Conditions 1(1-4) and $a_{1}<a_{-1}$, then its image $F^{n}(f, u ; p ; \zeta)$ is $A_{r}$-holomorphic (that is locally analytic) by $p$ in the domain $\left\{z \in A_{r}: a_{1}<\operatorname{Re}(z)<a_{-1}\right\}$, as well as by $\zeta \in A_{r}$, where $1 \leq r \in N, 2^{r-1} \leq n \leq 2^{r}-1$, the function $u(p, t ; \zeta)$ is given by $1(8,8.1)$ or $2(1,2,2.1)$.

Proof. At first consider the characteristic functions $\chi_{U_{v}}(t)$, where $\chi_{U}(t)=1$ for each $t \in U$, while $\chi_{U}(t)=0$ for every $t \in R^{n} \backslash U$,

$U_{v}:=\left\{t \in R^{n}: v_{j} t_{j} \geq 0 \forall j=1, \cdots, n\right\}$ is the domain in the Euclidean space $R^{n}$ for any $v$ from $\S 1$. Therefore,

1) $F_{u}^{n}(p ; \zeta):=$

$$
\sum_{\left[v=\left(v_{1}, \cdots, v_{n}\right): v_{1}, \cdots, v_{n} \in\{-1,1\}\right]} \int_{U_{v}} f(t) \exp (-u(p, t ; \zeta)) \mathrm{d} t,
$$

since $\lambda_{n}\left(U_{v} \cap U_{w}\right)=0$ for each $v \neq w$. Each integral $\int_{U_{v}} f(t) \exp (-u(p, t ; \zeta)) \mathrm{d} t$ is absolutely convergent for each $p \in A_{r}$ with the real part $a_{1}<\operatorname{Re}(p)<a_{-1}$, since it is majorized by the converging integral

2) $\left|\int_{U_{v}} f(t) \exp (-u(p, t ; \zeta)) \mathrm{d} t\right| \leq \int_{0}^{\infty} \cdots \int_{0}^{\infty} C_{v} \exp \left\{-v_{1}\left(w-a_{v_{1}}\right) y_{1}-\cdots-v_{n}\left(w-a_{v_{n}}\right) y_{n}-\zeta_{0}\right\} \mathrm{d} y_{1} \cdots \mathrm{d} y_{n}$

$$
=C_{v} e^{-\zeta_{0}} \prod_{j=1}^{n} v_{j}\left(w-a_{v_{j}}\right)^{-1},
$$

where $w=\operatorname{Re}(p)$, since $\left|e^{z}\right|=\exp (\operatorname{Re}(z))$ for each $z \in A_{r}$ in view of Corollary 3.3 [6]. While an integral, produced from the integral (1) differentiating by $p$ converges also uniformly:

$$
\text { 3) } \begin{aligned}
\left|\int_{U_{v}} f(t)[\partial \exp (-u(p, t ; \zeta)) / \partial p] \cdot h \mathrm{~d} t\right| \\
\leq \int_{0}^{\infty} \cdots \int_{0}^{\infty} C_{v}\left|\left(h_{0}\left(v_{1} y_{1}+\cdots+v_{n} y_{n}\right), h_{1}\left(v_{1} y_{1}+\cdots+v_{n} y_{n}\right), \cdots, h_{n-1}\left(v_{n-1} y_{n-1}+v_{n} y_{n}\right), h_{n} v_{n} y_{n}\right)\right| \\
\quad \exp \left\{-v_{1}\left(w-a_{v_{1}}\right) y_{1}-\ldots-v_{n}\left(w-a_{v_{n}}\right) y_{n}-\zeta_{0}\right\} \mathrm{d} y_{1} \cdots \mathrm{d} y_{n}
\end{aligned}
$$

for each $h \in A_{r}$, since each $z \in A_{r}$ can be written in the form $z=|z| \exp (M)$, where $|z|^{2}=z \tilde{z} \in[0, \infty) \subset R$, $M \in A_{r}, \operatorname{Re}(M):=(M+\tilde{M}) / 2=0$ in accordance with Proposition 3.2 [6]. In view of Equations 2(5,6):
4) $\partial\left(\int_{R^{n}} f(t) \exp (-u(p, t ; \zeta)) \mathrm{d} t\right) / \partial \tilde{p}=0$ and

5) $\partial\left(\int_{R^{n}} f(t) \exp (-u(p, t ; \zeta)) \mathrm{d} t\right) / \partial \tilde{\zeta}=0$, while

6) $\left|\int_{U_{v}} f(t)[\partial \exp (-u(p, t ; \zeta)) / \partial \zeta] \cdot h \mathrm{~d} t\right|$

$$
\leq|h| \int_{0}^{\infty} \cdots \int_{0}^{\infty} C_{v} \exp \left\{-v_{1}\left(w-a_{v_{1}}\right) y_{1}-\cdots-v_{n}\left(w-a_{v_{n}}\right) y_{n}-\zeta_{0}\right\} \mathrm{d} y_{1} \cdots \mathrm{d} y_{n}=|h| C_{v} e^{-\zeta_{0}} \prod_{j=1}^{n} v_{j}\left(w-a_{v_{j}}\right)^{-1}
$$


for each $h \in A_{r}$. In view of convergence of integrals given above (1-6) the multiparameter non-commutative transform $F_{u}^{n}(p ; \zeta)$ is (super)differentiable by $p$ and $\zeta$, moreover, $\partial F_{u}^{n}(p ; \zeta) / \partial \tilde{p}=0$ and

$\partial F_{u}^{n}(p ; \zeta) / \partial \tilde{\zeta}=0$ in the considered $(p, \zeta)$-representation. In accordance with $[5,6]$ a function $g(p)$ is locally analytic by $p$ in an open domain $U$ in the Cayley-Dickson algebra $A_{r}, 2 \leq r$, if and only if it is (super)differentiable by $p$, in another words $A_{r}$-holomorphic. Thus, $F_{u}^{n}(p ; \zeta)$ is $A_{r}$-holomorphic by $p \in A_{\text {, with }} a_{1}<\operatorname{Re}(p)<a_{-1}$ and $\zeta \in A_{r}$ due to Theorem 2.6 [4].

\section{Corollary}

Let suppositions of Theorem 4 be satisfied. Then the image $F^{n}(f, u ; p ; \zeta)$ with $u=u(p, t ; \zeta)$ given by 2 $(1,2)$ has the following periodicity properties:

1) for each $j=1, \cdots, n$ and $\beta \in 2 \pi Z$;

2) for each $j=1, \cdots, n-1$ so that $\zeta_{0}^{1}=\zeta_{0}^{2}$ and $\zeta_{j}^{1}=-\zeta_{j}^{2}, \quad \zeta_{j+1}^{1}=\pi+\zeta_{j+1}^{2}, \quad \zeta_{s}^{1}=\zeta_{s}^{2}$ for each $s \neq j$ and $s \neq j+1$, while either $p_{j}^{1}=-p_{j}^{2}$ and $p_{l}^{1}=p_{l}^{2}$ for each $l \neq j$ with $\kappa=2$ or $p^{1}=p^{2}$ and $f(t)$ is an even function with $\kappa=2$ by the $s_{j}=\left(t_{j}+\cdots+t_{n}\right)$ variable or an odd function by $s_{j}=\left(t_{j}+\cdots+t_{n}\right)$ with $\kappa=1$;

3) $F^{n}\left(f, u ; p ; \zeta+\pi i_{1}\right)=-F^{n}(f, u ; p ; \zeta)$.

Proof. In accordance with Theorem 4 the image $F^{n}(f, u ; p ; \zeta)$ exists for each $p \in W_{f}:=\left\{z \in A_{r}: a_{1}<\operatorname{Re}(z)<a_{-1}\right\}$ and $\zeta \in A_{r}$, where $1 \leq r$. Then from the $2 \pi$ periodicity of sine and cosine functions the first statement follows. From $\sin (-\phi)=-\sin (\phi), \cos (\phi)=\cos (-\phi)$, $\sin (\pi+\phi)=-\sin (\phi), \quad \cos (\phi+\pi)=-\cos (\phi) \quad$ we get that $\cos \left(p_{j} s_{j}+\zeta_{j}^{1}\right)=\cos \left(-p_{j} s_{j}+\zeta_{j}^{2}\right)$,

$$
\begin{aligned}
& \sin \left(p_{j} s_{j}+\zeta_{j}^{1}\right) \cos \left(p_{j+1} s_{j+1}+\zeta_{j+1}^{1}\right) \\
& =\left(-\sin \left(-p_{j} s_{j}+\zeta_{j}^{2}\right)\right)\left(-\cos \left(p_{j+1} s_{j+1}+\zeta_{j+1}^{2}\right)\right)
\end{aligned}
$$

and

$$
\begin{aligned}
& \sin \left(p_{j} s_{j}+\zeta_{j}^{1}\right) \sin \left(p_{j+1} s_{j+1}+\zeta_{j+1}^{1}\right) \\
& =\left(-\sin \left(-p_{j} s_{j}+\zeta_{j}^{2}\right)\right)\left(-\sin \left(p_{j+1} s_{j+1}+\zeta_{j+1}^{2}\right)\right)
\end{aligned}
$$

for each $t \in R^{n}$. On the other hand, either $p_{j}^{1}=-p_{j}^{2}$ and $p_{l}^{1}=p_{l}^{2}$ for each $l \neq j \geq 1$ with $\kappa=2$ or $p^{1}=p^{2}$ and

$$
\begin{aligned}
& f\left(t_{1}, \cdots, s_{j-1}+s_{j},-s_{j}-s_{j+1}, t_{j+1}, \cdots, t_{n}\right) \\
& =(-1)^{\kappa} f\left(t_{1}, \cdots, s_{j-1}-s_{j}, s_{j}-s_{j+1}, t_{j+1}, \cdots, t_{n}\right)
\end{aligned}
$$

is an even with $\kappa=2$ or odd with $\kappa=1$ function by the $s_{j}=\left(t_{j}+\cdots+t_{n}\right)$ variable for each $t=\left(t_{1}, \cdots, t_{n}\right) \in R^{n}$, where $t_{j}=s_{j}-s_{j+1}$ for $j=1, \cdots, n, s_{n+1}=s_{n+1}(n ; t)=0$. From this and Formulas $2(1,2,4)$ the second and the third statements of this corollary follow.

\subsection{Remark}

For a subset $U$ in $A_{r}$ we put

$$
\pi_{s, p, t}(U):=\left\{u: z \in U, z=\sum_{v \in b} w_{v} v, u=w_{s} s+w_{p} p\right\}
$$

for each $s \neq p \in b$, where

$$
\begin{aligned}
& t:=\sum_{v \in b \backslash\{s, p\}} w_{v} v \in A_{r, s, p}:= \\
& \left\{z \in A_{r}: z=\sum_{v \in b} w_{v} v, w_{s}=w_{p}=0, w_{v} \in R \forall v \in b\right\},
\end{aligned}
$$

where $b:=\left\{i_{0}, i_{1}, \cdots, i_{2^{r}-1}\right\}$ is the family of standard generators of the Cayley-Dickson algebra $A_{r}$. That is, geometrically $\pi_{s, p, t}(U)$ means the projection on the complex plane $C_{s, p}$ of the intersection $U$ with the plane $\tilde{\pi}_{s, p, t} \ni_{t} t, C_{s, p}:=\{a s+b p: a, b \in R\}$, since

$s p+\hat{b}:=b \backslash\{1\}$. Recall that in $\S \S 2.5-7$ [6] for each continuous function $f: U \rightarrow A_{r}$ it was defined the operator $\hat{f}$ by each variable $z \in A_{r}$. For the non-commutative integral transformations consider, for example, the left algorithm of calculations of integrals.

A Hausdorff topological space $X$ is said to be $n$ connected for $n \geq 0$ if each continuous map

$f: S^{k} \rightarrow X$ from the $k$-dimensional real unit sphere into $X$ has a continuous extension over $R^{k+1}$ for each $k \leq n$ (see also [17]). A 1-connected space is also said to be simply connected.

It is supposed further, that a domain $U$ in $A_{r}$ has the property that $U$ is $\left(2^{r}-1\right)$-connected; $\pi_{s, p, t}(U)$ is simply connected in $C$ for each $k=0,1, \cdots, 2^{r-1}$, $s=i_{2 k}, \quad p=i_{2 k+1}, \quad t \in A_{r, s, p}$ and $u \in C_{s, p}$, for which there exists $z=u+t \in U$.

\subsection{Theorem}

If a function $f(t)$ is an original (see Definition 1), such that ${ }_{N} F_{u}^{n}(p ; \zeta)$ is its image multiparameter noncommutative transform, where the functions $f$ and $F_{u}^{n}$ are written in the forms given by 3(3,3.1), $f\left(R^{n}\right) \subset A_{r}$ over the Cayley-Dickson algebra $A_{r}$, where $1 \leq r \in N$, $2^{r-1} \leq n \leq 2^{r}-1$.

Then at each point $t$, where $f(t)$ satisfies the Hölder condition the equality is accomplished:

$$
\text { 1) } \begin{aligned}
f(t) & =\left\{\left[\left(2 \pi N_{n}\right)^{-1} \int_{-N_{n} \infty}^{N_{n} \infty}\right]\left(\cdots\left(\left[\left(2 \pi N_{1}\right)^{-1} \int_{-N_{1} \infty}^{N_{1} \infty}\right]{ }_{N} F_{u}^{n}(a+p ; \zeta) \exp \{u(a+p, t ; \zeta)\}\right) \cdots\right) \mathrm{d} p\right\} \\
= & :\left(F^{n}\right)^{-1}\left({ }_{N} F_{u}^{n}(a+p ; \zeta), u, t ; \zeta\right),
\end{aligned}
$$


where either $u(p, t ; \zeta)=\langle p, t)+\zeta$ or $u(p, t ; \zeta)=p_{0} s_{1}+M_{N}(p, t ; \zeta)+\zeta_{0}$ (see $\S \S 1$ and 2$)$, the integrals are taken along the straight lines $p\left(\tau_{j}\right)=N_{j} \tau_{j} \in A_{r}, \tau_{j} \in R$ for each $j=1, \cdots, n$; $a_{1}<\operatorname{Re}(p)=a<a_{-1}$ and this integral is understood in the sense of the principal value, $t=\left(t_{1}, \cdots, t_{n}\right) \in R^{n}$,

$$
d p=\left(\cdots\left(\left(d\left[p_{1} N_{1}\right]\right) d\left[p_{2} N_{2}\right]\right) \ldots\right) d\left[p_{n} N_{n}\right] .
$$

Proof. In Integral (1) an integrand $\eta(p) d p$ certainly corresponds to the iterated integral as $\left(\cdots\left(\eta(p) d\left[p_{1} N_{1}\right]\right) \cdots\right) d\left[p_{n} N_{n}\right]$, where $p=p_{1} N_{1}+\cdots+p_{n} N_{n}, \quad p_{1}, \cdots, p_{n} \in R$. Using Decomposition 3(3.1) of a function $f$ it is sufficient to consider the inverse transformation of the real valued function $f_{j}$, which we denote for simplicity by $f$. We put ${ }_{N} F_{u, j}^{n}(p ; \zeta):=\int_{R^{n}} f_{j}(t) \exp (-u(p, t ; \zeta)) \mathrm{d} t$.

If $\eta$ is a holomorphic function of the CayleyDickson variable, then locally in a simply connected domain $U$ in each ball $B\left(A_{r}, z_{0}, R\right)$ with the center at $z_{0}$ of radius $R>0$ contained in the interior $\operatorname{Int}(U)$ of the domain $U$ there is accomplished the equality $\left(\partial\left[\int_{z_{0}}^{z} \eta(a+\zeta) \mathrm{d} \zeta\right] / \partial z\right) .1=\eta(a+z)$, where the integral depends only on an initial $z_{0}$ and a final $z$ points of a rectifiable path in $B\left(A_{r}, z_{0}, R\right), a \in R$ (see also Theorem 2.14 [4]). Therefore, along the straight line $N_{j} R$ the restriction of the antiderivative has the form $\int_{\theta_{0}}^{\theta} \eta\left(a+N_{j} \tau_{j}\right) \mathrm{d} \tau_{j}$, since

2) $\int_{z_{0}=N_{j} \theta_{0}}^{z=N_{j} \theta} \eta(a+\zeta) \mathrm{d} \zeta=\int_{\theta_{0}}^{\theta} \hat{\eta}\left(a+N_{j} \tau_{j}\right) \cdot N_{j} \mathrm{~d} \tau_{j}$, where $\partial \eta(a+z) / \partial \theta=(\partial \eta(a+z) / \partial z) . \quad N_{j}$ for the (super)differentiable by $z \in U$ function $\eta(z)$, when $z=\theta N_{j}, \theta \in R$. For the chosen branch of the line integral specified by the left algorithm this antiderivative is unique up to a constant from $A_{r}$ with the given $z$-representation $v$ of the function $\eta$ [4-6]. On the other hand, for analytic functions with real expansion coefficients in their power series non-commutative integrals specified by left or right algorithms along straight lines coincide with usual Riemann integrals by the corresponding variables. The functions $\sin (z), \cos (z)$ and $e^{z}$ participating in the multiparameter non-commutative transform are analytic with real expansion coefficients in their series by powers of $z \in A_{r}$.

Using Formula 4(1) we reduce the consideration to $\chi_{U_{v}}(t) f(t)$ instead of $f(t)$. By symmetry properties of such domains and integrals and utilizing change of variables it is sufficient to consider $U_{v}$ with $v=(1, \cdots, 1)$. In this case $\int_{R^{n}}$ for the direct multi- parameter non-commutative transform 1(7) and 2(4) reduces to $\int_{0}^{\infty} \cdots \int_{0}^{\infty}$. Therefore, we consider in this proof below the domain $U_{1, \cdots, 1}$ only. Using Formulas $3(3,3.1)$ and $2(1,2,2.1)$ we mention that any real algebra with generators $N_{0}=1, \quad N_{k}$ and $N_{j}$ with $1 \leq k \neq j$ is isomorphic with the quaternion skew field $H$, since $\operatorname{Re}\left(N_{j} N_{k}\right)=0$ and $\left|N_{j}\right|=1,\left|N_{k}\right|=1$ and $\left|N_{j} N_{k}\right|=1$. Then

$$
\exp (\alpha+M \beta) \exp (\gamma+M \omega)=\exp ((\alpha+\gamma)+M(\beta+\omega))
$$

for each real numbers $\alpha, \beta, \gamma, \delta$ and a purely imaginary Cayley-Dickson number $M$. The octonion algebra $O$ is alternative, while the real field $R$ is the center of the Cayley-Dickson algebra $A_{r}$. We consider the integral

3) $g_{b}(t):=\left[\left(2 \pi N_{n}\right)^{-1} \int_{-N_{n} b}^{N_{n} b}\right]\left(\cdots\left(\left[\left(2 \pi N_{1}\right)^{-1} \int_{-N_{1} b}^{N_{1} b}\right]{ }_{N} F_{u, j}^{n}(a+p ; \zeta) \exp \{u(a+p, t ; \zeta)\}\right) \cdots\right) \mathrm{d} p$

for each positive value of the parameter $0<b<\infty$. With the help of generators of the Cayley-Dickson algebra $A_{r}$ and the Fubini Theorem for real valued components of the function the integral can be written in the form:

4) $g_{b}(t)=\left[\left(2 \pi N_{n}\right)^{-1} \int_{0}^{\infty} \mathrm{d} \tau_{n} \int_{-N_{n} b}^{N_{n} b}\right]\left(\cdots\left(\left[\left(2 \pi N_{1}\right)^{-1} \int_{0}^{\infty} \mathrm{d} \tau_{1} \int_{-N_{1} b}^{N_{1} b}\right] f(\tau) \exp \left\{-u_{N}(a+p, t ; \zeta)\right\} \exp \left\{u_{N}(a+p, \tau ; \zeta)\right\}\right) \cdots\right) \mathrm{d} p$,

since the integral $\int_{U_{1, \ldots, 1}} f(\tau) \exp \left\{-u_{N}(a+p, \tau ; \zeta)\right\} \mathrm{d} \tau$ for any marked $0<\delta<\left(a_{-1}-a_{1}\right) / 3$ is uniformly converging relative to $p$ in the domain

$a_{1}+\delta \leq \operatorname{Re}(p) \leq a_{-1}-\delta$ in $A_{r}$ (see also Proposition 2.18 [4]). If take marked $t_{k}$ for each $k \neq j$ and $S=N_{j}$ for some $j \geq 1$ in Lemma 2.17 [4] considering the variable $t_{j}$, then with a suitable ( $R$-linear) automorphism $v$ of the Cayley-Dickson algebra $A_{r}$ an expression for $v(M(p, t ; \zeta))$ simplifies like in the complex case with $C_{K}:=R \oplus R K$ for a purely imagi- nary Cayley-Dickson number $K,|K|=1$, instead of $C:=R \oplus R i_{1}$, where $v(x)=x$ for each real number $x \in R$. But each equality $\alpha=\beta$ in $A_{r}$ is equivalent to $v(\alpha)=v(\beta)$. Then

$$
\text { 5) } \operatorname{Re}\left[\left(N_{j} N_{q}\right)\left(N_{j} N_{l}\right)^{*}\right]=\operatorname{Re}\left(N_{q} N_{l}^{*}\right)=\delta_{q, l}
$$

for each $q, l$.

If $S^{j}=\sum_{0 \leq l \leq n: l \neq j} \alpha_{l} N_{l}, \quad N^{j}=\sum_{0 \leq l \leq n ; l \neq j} \beta_{l} N_{l} \quad$ with $j \geq 1$ and real numbers $\alpha_{l}, \beta_{l} \in R$ for each $l$, then

6) $\operatorname{Re}\left[\left(N_{j} S^{j}\right)\left(N_{j} N^{j}\right)^{*}\right]=\operatorname{Re}\left[S^{j}\left(N^{j}\right)^{*}\right]=\sum_{l} \alpha_{l} \beta_{l}$. 
The latter identity can be applied to either

$$
S^{k}=M_{k+1}\left(p_{k+1} N_{k+1}+\cdots+p_{n} N_{n},\left(t_{k+1}, \cdots, t_{n}\right) ; \zeta_{k+1} N_{k+1}+\cdots+\zeta_{n} N_{n}\right)
$$

and

$$
N^{k}=M_{k+1}\left(p_{k+1} N_{k+1}+\cdots+p_{n} N_{n},\left(\tau_{k+1}, \cdots, \tau_{n}\right) ; \zeta_{k+1} N_{k+1}+\cdots+\zeta_{n} N_{n}\right),
$$

or

$$
S^{k}=\left(p_{k+1} t_{k+1}+\zeta_{k+1}\right) N_{k+1}+\cdots+\left(p_{n} t_{n}+\zeta_{n}\right) N_{n}
$$

and

$$
N^{k}=\left(p_{k+1} \tau_{k+1}+\zeta_{k+1}\right) N_{k+1}+\cdots+\left(p_{n} \tau_{n}+\zeta_{n}\right) N_{n},
$$

where

7) $M_{k+1}\left(p_{k+1} N_{k+1}+\ldots+p_{n} N_{n},\left(t_{k+1}, \cdots, t_{n}\right) ; \zeta_{k+1} N_{k+1}+\ldots+\zeta_{n} N_{n}\right)$,

$$
=\left(p_{k+1} s_{1, k+1}+\zeta_{k+1}\right)+N_{n}\left[\sin \left(p_{k+2} s_{2, k+1}+\zeta_{k+2}\right) \cdots \sin \left(p_{n} s_{n-k, k+1}+\zeta_{n}\right)\right]
$$

8) $s_{j, k+1}=s_{j, k+1}(n ; t)=t_{k+j}+\cdots+t_{n}=s_{k+j}(n ; t)$

$$
s_{k}(n ; \tau)-s_{j}(n ; \tau)=s_{k}(j-1 ; \tau)=\tau_{k}+\cdots+\tau_{j-1}
$$

for each $j=1, \cdots, n-1 ; s_{n-k, k+1}=s_{n-k, k+1}(n ; t)=t_{n}$. We for each $1 \leq k<j \leq n$. By our convention take the limit of $g_{b}(t)$ when $b$ tends to the infinity. $s_{k}(n ; \tau)=s_{1}(n ; \tau)$ for $k<1$, while $s_{k}(n ; \tau)=0$ for Evidently, $k>n$. Put

9) $u_{n, j}\left(p_{0}+p_{j} N_{j}+\cdots+p_{n} N_{n},\left(\tau_{j}, \cdots, \tau_{n}\right) ; \zeta_{0}+\zeta_{j} N_{j}+\cdots+\zeta_{n} N_{n}\right)$

$$
=\zeta_{0}+p_{0} s_{1, j}+M_{j}\left(p_{j} N_{j}+\cdots+p_{n} N_{n},\left(\tau_{j}, \cdots, \tau_{n}\right) ; \zeta_{0}+\zeta_{j} N_{j}+\cdots+\zeta_{n} N_{n}\right)
$$

for $u_{N}$ given by 2(1,2,2.1), where $M_{j}$ is prescribed by (7), $s_{k, j}=s_{k, j}(n ; \tau)$;

10) $u_{n, j}\left(p_{0}+p_{j} N_{j}+\cdots+p_{n} N_{n},\left(\tau_{j}, \cdots, \tau_{n}\right) ; \zeta_{0}+\zeta_{j} N_{j}+\cdots+\zeta_{n} N_{n}\right)=\zeta_{0}+p_{0} s_{1, j}+\sum_{k=j}^{n}\left(p_{k} \tau_{k}+\zeta_{k}\right) N_{k}$

for $u=u_{N}$ given by $1(8,8.1)$. For $j>1$ the parameter $\zeta_{0}$ for $u=u_{N}$ given by $1(8,8.1)$ or $2(1,2,2.1)$ can be taken equal to zero.
When $t_{1}, \cdots, t_{j-1}, t_{j+1}, \cdots, t_{n}$ and

$p_{1}, \cdots, p_{j-1}, p_{j+1}, \cdots, p_{n}$ variables are marked, we take the parameter

$$
\begin{aligned}
\zeta^{j} & :=\zeta^{j}\left(p_{j} N_{j}+\cdots+p_{n} N_{n},\left(\tau_{j}, \cdots, \tau_{n}\right) ; \zeta_{0}+\zeta_{j} N_{j}+\cdots+\zeta_{n} N_{n}\right) \\
& :=\left(\zeta_{0}+\zeta_{j} N_{j}+\cdots+\zeta_{n} N_{n}\right)+\left(a+p_{0}\right) s_{j+1}+p_{j+1} s_{j+1} N_{j+1}+\cdots+p_{n} s_{n} N_{n}
\end{aligned}
$$

for $u(p, \tau ; \zeta)$ given by Formulas 2(1,2,2.1) or

$$
\begin{aligned}
\zeta^{j} & :=\zeta^{j}\left(p_{j} N_{j}+\cdots+p_{n} N_{n},\left(\tau_{j}, \cdots, \tau_{n}\right) ; \zeta_{0}+\zeta_{j} N_{j}+\cdots+\zeta_{n} N_{n}\right) \\
& :=\left(\zeta_{0}+\zeta_{j} N_{j}+\cdots+\zeta_{n} N_{n}\right)+\left(a+p_{0}\right) s_{j+1}+p_{j+1} \tau_{j+1} N_{j+1}+\cdots+p_{n} \tau_{n} N_{n}
\end{aligned}
$$

for $u(p, \tau ; \zeta)$ described in $1(8,8.1)$. Then the integral operator

$$
\lim _{b \rightarrow \infty}\left[\left(2 \pi N_{j}\right)^{-1} \int_{0}^{\infty} \mathrm{d} \tau_{j} \int_{-N_{j} b}^{N_{j} b}\right] \cdots\left(\mathrm{d} p_{j} N_{j}\right)
$$

(see also Formula (4) above) applied to the function

$$
\begin{aligned}
& f\left(t_{1}, \cdots, t_{j-1}, \tau_{j}, \cdots, \tau_{n}\right) \exp \left\{-u_{N, j}\left(a+p_{0}+p_{j} N_{j}+\cdots+p_{n} N_{n},\left(t_{j}, \cdots, t_{n}\right) ; \zeta_{0}+\zeta_{j} N_{j}+\cdots+\zeta_{n} N_{n}\right)\right\} \\
& \exp \left\{u_{N, j}\left(a+p_{0}+p_{j} N_{j}+\cdots+p_{n} N_{n},\left(\tau_{j}, \cdots, \tau_{n}\right) ; \zeta_{0}+\zeta_{j} N_{j}+\cdots+\zeta_{n} N_{n}\right)\right\}
\end{aligned}
$$


with the parameter $\zeta^{j}$ instead of $\zeta$ treated by Theorems 2.19 and 3.15 [4] gives the inversion formula corresponding to the real variable $t_{j}$ for $f(t)$ and to the Cayley-Dickson variable $p_{0} N_{0}+p_{j} N_{j}$ restricted on the complex plane $C_{N_{j}}=R \oplus R N_{j}$, since

$d\left(\tau_{j}+c\right)=d \tau_{j}$ for each (real) constant $c$. After integrations with $j=1, \cdots, k$ with the help of Formulas (6$10)$ and $3(1,2)$ we get the following:

$$
\text { 11) } \begin{aligned}
\lim _{b \rightarrow \infty} g_{b}(t)=\operatorname{Re}\left[\left(2 \pi N_{n}\right)^{-1} \int_{0}^{\infty} \mathrm{d} \tau_{n} \int_{-N_{n} \infty}^{N_{n} \infty}\right]\left(\cdots \left(\left[\left(2 \pi N_{k+1}\right)^{-1} \int_{0}^{\infty} \mathrm{d} \tau_{k+1} \int_{-N_{k+1} \infty}^{N_{k+1} \infty}\right]\right.\right. \\
f\left(t_{1}, \cdots, t_{k}, \tau_{k+1}, \cdots, \tau_{n}\right) \exp \left\{-u_{N, k+1}\left(\left(a+p_{0}+p_{k+1} N_{k+1}+\cdots+p_{n} N_{n}\right),\left(t_{k+1}, \cdots, t_{n}\right) ;\left(\zeta_{0}+\zeta_{k+1} N_{k+1}+\cdots+\zeta_{n} N_{n}\right)\right)\right\} \\
\left.\left.\left.\exp \left\{u_{N, k+1}\left(\left(a+p_{0}+p_{k+1} N_{k+1}+\ldots+p_{n} N_{n}\right),\left(\tau_{k+1}, \cdots, \tau_{n}\right) ;\left(\zeta_{0}+\zeta_{k+1} N_{k+1}+\cdots+\zeta_{n} N_{n}\right)\right)\right\}\right)\right) \cdots\right) \mathrm{d} p .
\end{aligned}
$$

Moreover, $\operatorname{Re}\left(f_{q}\right)=f_{q}$ for each $q$ and in (11) the function $f=f_{q}$ stands for some marked $q$ in accordance with Decompositions 3(3,3.1) and the beginning of this proof.

Mention, that the algebra $\operatorname{alg}_{R}\left(N_{j}, N_{k}, N_{l}\right)$ over the real field with three generators $N_{j}, N_{k}$ and $N_{l}$ is alternative. The product $N_{k} N_{l}$ of two generators is also the corresponding generator $(-1)^{\xi(k, l)} N_{m}$ with the definite number $m=m(k, l)$ and the sign multiplier $(-1)^{\xi(k, l)}$, where $\xi(k, l) \in\{0,1\}$. On the other hand,
$N_{k_{1}}\left[\tilde{N}_{j}\left(N_{j}\left(N_{k_{2}} N_{l}\right)\right)\right]=N_{k_{1}}\left(N_{k_{2}} N_{l}\right)$. We use decompositions (7-10) and take $k_{2}=l$ due to Formula (11), where $R e$ stands on the right side of the equality, since $\operatorname{Re}\left(N_{k} N_{l}\right)=0$ and $\operatorname{Re}\left[\tilde{N}_{j}\left(N_{j}\left(N_{k} N_{l}\right)\right)\right]=0$ for each $k \neq l$. Thus the repeated application of this procedure by $j=1,2, \cdots, n$ leads to Formula (1) of this theorem.

\section{Corollary}

If the conditions of Theorem 6 are satisfied, then

1) $f(t)=(2 \pi)^{-n} \int_{R^{n}} F_{u}^{n}(a+p ; \zeta) \exp \{u(a+p, t ; \zeta)\} \mathrm{d} p_{1} \cdots \mathrm{d} p_{n}=\left(F^{n}\right)^{-1}\left({ }_{N} F_{u}^{n}(a+p ; \zeta), u, t ; \zeta\right)$.

Proof. Each algebra $\operatorname{alg}_{R}\left(N_{j}, N_{k}, N_{l}\right)$ is alternative. Therefore, in accordance with $\S 6$ and Formulas
$1(8,8.1)$ and 2(1-4) for each non-commutative integral given by the left algorithm we get

2) $N_{j}^{-1} \int_{-N_{j} b}^{N_{j} b}\left[f(\tau) \exp \left\{-u_{N}(a+p, t ; \zeta)\right\}\right] \exp \left\{u_{N}(a+p, \tau ; \zeta)\right\} \mathrm{d}\left(p_{j} N_{j}\right)$

$$
\begin{aligned}
& \sum_{l=0}^{2^{r}-1} \tilde{N}_{j}\left[N_{j}\left(\int_{-N_{j} b}^{N_{j} b}\left[N_{l} f_{l}(\tau) \exp \left\{-u_{N}(a+p, t ; \zeta)\right\}\right] \exp \left\{u_{N}(a+p, \tau ; \zeta)\right\} \mathrm{d} p_{j}\right)\right] \\
& =\int_{-b}^{b}\left[f(\tau) \exp \left\{-u_{N}(a+p, t ; \zeta)\right\}\right] \exp \left\{u_{N}(a+p, \tau ; \zeta)\right\} \mathrm{d} p_{j}
\end{aligned}
$$

for each $j=1, \cdots, n$, since the real field is the center of the Cayley-Dickson algebra $A_{r}$, while the functions $\sin$ and $\cos$ are analytic with real expansion coefficients. Thus

3) $g_{b}(t)=(2 \pi)^{-n}\left[\int_{0}^{\infty} \mathrm{d} \tau_{n} \int_{-b}^{b}\right]\left(\cdots\left(\left[\int_{0}^{\infty} \mathrm{d} \tau_{1} \int_{-b}^{b}\right] f(\tau) \exp \left\{-u_{N}(a+p, t ; \zeta)\right\} \exp \left\{u_{N}(a+p, \tau ; \zeta)\right\}\right) \cdots\right) \mathrm{d} p_{1} \cdots \mathrm{d} p_{n}$

hence taking the limit with $b$ tending to the infinity implies, that the non-commutative iterated (multiple) integral in Formula 6(1) reduces to the principal value of the usual integral by real variables $\left(\tau_{1}, \cdots, \tau_{n}\right)$ and $\left(p_{1}, \cdots, p_{n}\right)$ 6.1(1).

\subsection{Theorem}

An original $f(t)$ with $f\left(R^{n}\right) \subset A_{r}$ over the CayleyDickson algebra $A_{r}$ with $1 \leq r \in N$ is completely defined by its image ${ }_{N} F_{u}^{n}(p ; \zeta)$ up to values at points of discontinuity, where the function $u(p, t ; \zeta)$ is given by $1(8,8.1)$ or $2(1,2,2.1)$.

Proof. Due to Corollary 6.1 the value $f(t)$ at each point $t$ of continuity of $f(t)$ has the expression throughout ${ }_{N} F_{u}^{n}(p ; \zeta)$ prescribed by Formula 6.1(1). Moreover, values of the original at points of discontinuity do not influence on the image ${ }_{N} F_{u}^{n}(p ; \zeta)$, since on each bounded interval in $R$ by each variable $t_{j}$ a number of points of discontinuity is finite and by our supposition above the original function $f(t)$ is $\lambda_{n}-$ 
almost everywhere on $R^{n}$ continuous.

\subsection{Theorem}

Suppose that a function ${ }_{N} F_{u}^{n}(p ; \zeta)$ is analytic by the variable $p \in A_{r}$ in a domain

$W:=\left\{p \in A_{r}: a_{1}<\operatorname{Re}(p)<a_{-1}\right\}$, where $2 \leq r \in N$,

$2^{r-1} \leq n \leq 2^{r}-1, \quad f\left(R^{n}\right) \subset A_{r}$, either

$u(p, t ; \zeta)=\langle p, t)+\zeta$ or

$u(p, t ; \zeta):=p_{0} s_{1}+M(p, t ; \zeta)+\zeta_{0}$ (see $\S \S 1$ and 2$)$.

Let ${ }_{N} F_{u}^{n}(p ; \zeta)$ be written in the form

${ }_{N} F_{u}^{n}(p ; \zeta)={ }_{N} F_{u}^{n, 0}(p ; \zeta)+{ }_{N} F_{u}^{n, 1}(p ; \zeta)$, where

${ }_{N} F_{u}^{n, 0}(p ; \zeta)$ is holomorphic by $p$ in the domain
$a_{1}<\operatorname{Re}(p)$. Let also ${ }_{N} F_{u}^{n, 1}(p ; \zeta)$ be holomorphic by

$p$ in the domain $\operatorname{Re}(p)<a_{-1}$. Moreover, for each

$a>a_{1}$ and $b<a_{-1}$ there exist constants $C_{a}>0$,

$C_{b}>0$ and $\varepsilon_{a}>0$ and $\varepsilon_{b}>0$ such that

1) $\left|{ }_{N} F_{u}^{n, 0}(p ; \zeta)\right| \leq C_{a} \exp \left(-\varepsilon_{a}|p|\right)$ for each $p \in A_{r}$ with $\operatorname{Re}(p) \geq a$,

2) $\left|{ }_{N} F_{u}^{n, 1}(p ; \zeta)\right| \leq C_{b} \exp \left(-\varepsilon_{b}|p|\right)$ for each $p \in A_{r}$

with $\operatorname{Re}(p) \leq b$, the integral,

3) $\int_{-N_{n} \infty}^{N_{n} \infty} \cdots \int_{-N_{1} \infty}^{N_{1} \infty}{ }_{N} F_{u}^{n, k}(w+p ; \zeta) \mathrm{d} p$ converges abso-

lutely for $k=0$ and $k=1$ and each $a_{1}<w<a_{-1}$. Then ${ }_{N} F_{u}^{n}(w+p ; \zeta)$ is the image of the function,

$$
\text { 4) } \begin{aligned}
f(t) & =\left[(2 \pi)^{-1} \tilde{N}_{n} \int_{-N_{n} \infty}^{N_{n^{\infty}}}\right]\left(\cdots\left(\left[(2 \pi)^{-1} \tilde{N}_{1} \int_{-N_{1} \infty}^{N_{1} \infty}\right]{ }_{N} F_{u}^{n}(w+p ; \zeta) \exp \{u(w+p, t ; \zeta)\}\right) \cdots\right) \mathrm{d} p \\
& =\left(F^{n}\right)^{-1}\left(\left({ }_{N} F_{u}^{n}(w+p ; \zeta), u, t ; \zeta\right) .\right.
\end{aligned}
$$

Proof. For the function ${ }_{N} F_{u}^{n, 1}(p ; \zeta)$ we consider the substitution of the variable $p=-g,-a_{-1}<\operatorname{Re}(g)$. Thus the proof reduces to the consideration of

${ }_{N} F_{u}^{n, 0}(w+p ; \zeta)$. An integration by $d p$ in the iterated integral (4) is treated as in $\S 6$. Take marked values of variables $p_{1}, \cdots, p_{j-1}, p_{j+1}, \cdots, p_{n}$ and $t_{1}, \cdots, t_{j-1}, t_{j+1}, \cdots, t_{n}$, where $s_{k}=s_{k}(n ; \tau)$ for each $k=1, \cdots, n$ (see $\S 6$ also). For a given parameter

$$
\begin{aligned}
\zeta^{j}:= & \left(\zeta_{0}+\zeta_{j} N_{j}+\cdots+\zeta_{n} N_{n}\right)+\left(w+p_{0}\right) s_{j+1} \\
& +p_{j+1} s_{j+1} N_{j+1}+\cdots+p_{n} s_{n} N_{n}
\end{aligned}
$$

for $u(p, \tau ; \zeta)$ prescribed by Formulas 2(1,2,2.1) or

$$
\begin{aligned}
\zeta^{j}:= & \left(\zeta_{0}+\zeta_{j} N_{j}+\cdots+\zeta_{n} N_{n}\right)+\left(w+p_{0}\right) s_{j+1} \\
& +p_{j+1} \tau_{j+1} N_{j+1}+\cdots+p_{n} \tau_{n} N_{n}
\end{aligned}
$$

for $u(p, t ; \zeta)$ given by $1(8,8.1)$ instead of $\zeta$ and any non-zero Cayley-Dickson number $\beta \in A_{r}$ we have

$$
\lim _{\tau_{j} \rightarrow \infty}\left[\beta \tau_{j}+\zeta^{j}\right] /\left[\beta \tau_{j}+\zeta\right]=1 .
$$

For any locally $z$-analytic function $g(z)$ in a domain $U$ satisfying conditions of $\S 5$ the homotopy theo-rem for a non-commutative line integral over $A_{r}$, $2 \leq r$, is satisfied (see $[5,6]$ ). In particular if $U$ contains the straight line $w+R N_{j}$ and the path

$\gamma_{j}\left(t_{j}\right):=\zeta^{j}+t_{j} N_{j}$, then $\int_{-N_{j}{ }^{\infty}}^{N_{j} \infty} g(z) \mathrm{d} z=\int_{\gamma_{j}} g(w+z) \mathrm{d} z$, when $\hat{g}(z) \rightarrow 0$ while $|z|$ tends to the infinity, since $\left|\zeta^{j}\right|$ is a finite number (see Lemma 2.23 in [4]). We apply this to the integrand in Formula (4), since ${ }_{N} F_{u}^{n}(w+p ; \zeta)$ is locally analytic by $p$ in accordance with Theorem 4 and Conditions $(1,2)$ are satisfied.

Then the integral operator $\left[\left(2 \pi N_{j}\right)^{-1} \int_{-N_{j} \infty}^{N_{j} \infty}\right]$ on the $j$-th step with the help of Theorems 2.22 and 3.16 [4] gives the inversion formula corresponding to the real parameter $t_{j}$ for $f(t)$ and to the Cayley-Dickson variable $p_{0} N_{0}+p_{j} N_{j}$ which is restricted on the complex plane $C_{N_{j}}=R \oplus R N_{j}$ (see also Formulas $6(4,11$ ) above). Therefore, an application of this procedure by $j=1,2, \cdots, n$ as in $\S 6$ implies Formula (4) of this theorem. Thus there exist originals $f^{0}$ and $f^{1}$ for functions ${ }_{N} F_{u}^{n, 0}(p ; \zeta)$ and ${ }_{N} F_{u}^{n, 1}(p ; \zeta)$ with a choice of $w \in R$ in the common domain

$a_{1}<\operatorname{Re}(p)<a_{-1}$. Then $f=f^{0}+f^{1}$ is the original for ${ }_{N} F_{u}^{n}(p ; \zeta)$ due to the distributivity of the multiplication in the Cayley-Dickson algebra $A_{r}$ leading to the additivity of the considered integral operator in Formula (4).

\section{Corollary}

Let the conditions of Theorem 8 be satisfied, then

1) $f(t)=(2 \pi)^{-n} \int_{R^{n}{ }_{N}} F_{u}^{n}(w+p ; \zeta) \exp \{u(w+p, t ; \zeta)\} \mathrm{d} p_{1} \cdots \mathrm{d} p_{n}=\left(F^{n}\right)^{-1}\left({ }_{N} F_{u}^{n}(w+p ; \zeta), u, t ; \zeta\right)$.

Proof. In accordance with $\S \S 6$ and 6.1 each noncommutative integral given by the left algorithm reduces to the principal value of the usual integral by the corresponding real variable:

2) $(2 \pi)^{-1} \tilde{N}_{j} \int_{-N_{j} \infty}^{N_{j} \infty}{ }_{N} F_{u}^{n}(w+p ; \zeta) \exp \{u(w+p, t ; \zeta)\} \mathrm{d}\left(p_{j} N_{j}\right)=(2 \pi)^{-1} \int_{-\infty}^{\infty}{ }_{N} F_{u}^{n}(w+p ; \zeta) \exp \{u(w+p, t ; \zeta)\} \mathrm{d} p_{j}$ 
for each $j=1, \cdots, n$. Thus Formula 8(4) with the noncommutative iterated (multiple) integral reduces to Formula 8.1(1) with the principal value of the usual integral by real variables $\left(p_{1}, \cdots, p_{n}\right)$.

\subsection{Note}

In Theorem 8 Conditions $(1,2)$ can be replaced on

1) $\lim _{n \rightarrow \infty} \sup _{p \in C_{R(n)}}\|\hat{F}(p)\|=0$,

where $C_{R(n)}:=\left\{z \in A_{r}:|z|=R(n), a_{1}<\operatorname{Re}(z)<a_{-1}\right\}$ is a sequence of intersections of spheres with a domain $W$, where $R(n)<R(n+1)$ for each $n, \lim _{n \rightarrow \infty} R(n)=\infty$. Indeed, this condition leads to the accomplishment of the A r analog of the Jordan Lemma for each $r \geq 2$ (see also Lemma 2.23 and Remark 2.24 [4]).

Subsequent properties of quaternion, octonion and general $A_{r}$ multiparameter non-commutative analogs of the Laplace transform are considered below. We denote by:

2) $W_{f}=\left\{p \in A_{r}: a_{1}(f)<\operatorname{Re}(p)<a_{-1}(f)\right\}$ a domain of ${ }_{N} F_{u}^{n}(p ; \zeta)$ by the $p$ variable, where $a_{1}=a_{1}(f)$ and $a_{-1}=a_{-1}(f)$ are as in $\S 1$. For an original
3) $f(t) \chi_{U_{1}, \ldots 1}(t)$ we put

$$
W_{f}=\left\{p \in A_{r}: a_{1}(f)<\operatorname{Re}(p)\right\},
$$

that is $a_{-1}=\infty$. Cases may be, when either the left hyperplane $\operatorname{Re}(p)=a_{1}$ or the right hyperplane

$\operatorname{Re}(p)=a_{-1}$ is (or both are) included in $W_{f}$. It may also happen that a domain reduces to the hyperplane $W_{f}=\left\{p: \operatorname{Re}(p)=a_{1}=a_{-1}\right\}$.

\subsection{Proposition}

If images ${ }_{N} F_{u}^{n}(p ; \zeta)$ and ${ }_{N} G_{u}^{n}(p ; \zeta)$ of functions originals $f(t)$ and $g(t)$ exist in domains $W_{f}$ and $W_{g}$ with values in $A_{r}$, where the function $u(p, t ; \zeta)$ is given by $1(8,8.1)$ or $2(1,2,2.1)$, then for each

$\alpha, \beta \in A_{r}$ in the case $A_{2}=H$; as well as $f$ and $g$ with values in $R$ and each $\alpha, \beta \in A_{r}$ or $f$ and $g$ with values in $A_{r}$ and each $\alpha, \beta \in R$ in the case of $A_{r}$ with $r \geq 3$; the function $\alpha{ }_{N} F_{u}(p ; \zeta)+\beta{ }_{N} G_{u}(p ; \zeta)$ is the image of the function $\alpha f(t)+\beta g(t)$ in a domain $W_{f} \cap W_{g}$.

Proof. Since the transforms ${ }_{N} F_{u}^{n}(p ; \zeta)$ and ${ }_{N} G_{u}^{n}(p ; \zeta)$ exist, then the integral

$$
\int_{R^{n}}(\alpha f(t)+\beta g(t)) \exp (-u(p, t ; \zeta)) \mathrm{d} t=\int_{R^{n}} \alpha f(t) \exp (-u(p, t ; \zeta)) \mathrm{d} t+\int_{R^{n}} \beta g(t) \exp (-u(p, t ; \zeta)) \mathrm{d} t
$$

converges in the domain

$$
W_{f} \cap W_{g}=\left\{p \in A_{r}: \max \left(a_{1}(f), a_{1}(g)\right)<\operatorname{Re}(p)<\min \left(a_{-1}(f), a_{-1}(g)\right)\right\} .
$$

We have $t \in R^{n}, 2^{r-1} \leq n \leq 2^{r}-1$, while $R$ is the center of the Cayley-Dickson algebra $A_{r}$. The quaternion skew field $H$ is associative. Thus, under the imposed conditions the constants $\alpha, \beta$ can be carried out outside integrals.

\subsection{Theorem}

Let $\alpha=$ const $>0$, let also $F^{n}(p ; \zeta)$ be an image of an original function $f(t)$ with either $u=\langle p, t)+\zeta$ or $u$ given by Formulas $2(1,2)$ over the Cayley-Dickson algebra $A_{r}$ with $2 \leq r<\infty, 2^{r-1} \leq n \leq 2^{r}-1$. Then an image $F^{n}(p / \alpha ; \zeta) / \alpha^{n}$ of the function $f(\alpha t)$ exists.

Proof. Since

$p_{j} s_{j}+\zeta_{j}=p_{j}\left(s_{j}^{\prime} / \alpha\right)+\zeta_{j}=\left(p_{j} / \alpha\right) s_{j}^{\prime}+\zeta_{j}$ for each $j=1, \cdots, n$, where $s_{j} \alpha=s_{j}^{\prime}, s_{j}=s_{j}(n ; t)$, $s_{j}^{\prime}=s_{j}(n ; \tau), \quad \tau_{j}=\alpha t_{j}$ for each $j=1, \cdots, n$. Then changing of these variables implies:

$$
\begin{aligned}
\int_{R^{n}} f(\alpha t) e^{-u(p, t ; \zeta)} \mathrm{d} t & =\int_{R^{n}} f(\tau) e^{-u(p, \tau / \alpha ; \zeta)} \mathrm{d} \tau / \alpha^{n} \\
& =F^{n}(p / \alpha ; \zeta) / \alpha^{n}
\end{aligned}
$$

due to the fact that the real filed $R$ is the center $Z\left(A_{r}\right)$ of the Cayley-Dickson algebra $A_{r}$.

\subsection{Theorem}

Let $f(t)$ be a function-original on the domain $U_{1, \ldots, 1}$ such that $\partial f(t) / \partial t_{k}$ also for $k=j-1$ and $k=j$ satisfies Conditions 1(1-4). Suppose that $u(p, t ; \zeta)$ is given by $2(1,2,2.1)$ or $1(8,8.1)$ over the Cayley-Dickson algebra $A_{r}$ with $2 \leq r<\infty, 2^{r-1} \leq n \leq 2^{r}-1$. Then

1) $F^{n}\left(\left(\partial f(t) / \partial t_{j}\right) \chi_{U_{1, \cdots, 1}}(t), u ; p ; \zeta\right)=-F^{n-1 ; t^{j}}\left(f(t) \chi_{U_{1, \cdots, 1}}\left(t^{j}\right), u\left(p, t^{j} ; \zeta\right) ; p ; \zeta\right)$

$$
+\left[p_{0}+\sum_{k=1}^{j} p_{k} S_{e_{k}}\right] F^{n}\left(f(t) \chi_{U_{1, \cdots, 1}}(t), u ; p ; \zeta\right)
$$

in the $A_{r}$ spherical coordinates or 
1.1) $F^{n}\left(\left(\partial f(t) / \partial t_{j}\right) \chi_{U_{1, \cdots, 1}}(t), u ; p ; \zeta\right)=-F^{n-1 ; t^{j}}\left(f(t) \chi_{U_{1, \cdots, 1}}\left(t^{j}\right), u\left(p, t^{j} ; \zeta\right) ; p ; \zeta\right)$

$$
+\left[p_{0}+p_{j} S_{e_{j}}\right] F^{n}\left(f(t) \chi_{U_{1, \cdots, 1}}(t), u ; p ; \zeta\right)
$$

in the $A_{r}$ Cartesian coordinates in a domain $W=\left\{p \in A_{r}: \max \left(a_{1}(f), a_{1}\left(\partial f / \partial t_{j}\right)\right)<\operatorname{Re}(p)\right\}$, where $\quad k \geq 1$.

$t^{j}:=\left(t_{1}, \cdots, t_{j}, \cdots, t_{n}: t_{j}=0\right), \quad S_{e_{k}}=-\partial / \partial \zeta_{k}$ for each

Proof. Certainly,

2) $\partial f(t(s)) / \partial s_{1}=\partial f(t) / \partial t_{1}$ and

2.1) $\partial f(t) / \partial t_{j}=\sum_{k=1}^{n}\left(\partial f(t(s)) / \partial s_{k}\right)\left(\partial s_{k} / \partial t_{j}\right)=\sum_{k=1}^{j} \partial f(t(s)) / \partial s_{k}$

for each $j=2, \cdots, n$, since $t_{j}=s_{j}-s_{j+1}, t_{1}=s_{1}-s_{2}$,

Formulas $30(6,7)[4]$ we have the equality in the $A_{r}$ where $s_{j}=s_{j}(n ; t), s_{n+l}=0$ for each $l \geq 1$. From spherical coordinates:

3) $\partial \exp (-u(p, t ; \zeta)) / \partial s_{j}=-p_{0} \delta_{1, j} \exp (-u(p, t ; \zeta))-p_{j} S_{e_{j}} \exp (-u(p, t ; \zeta))$,

since $\exp (-u(p, t ; \zeta))=\exp \left\{-p_{0} s_{1}-\zeta_{0}\right\} \exp (-M(p, t ; \zeta))$,

$\partial \exp \left(-p_{0} s_{1}-\zeta_{0}\right) / \partial s_{j}=-p_{0} \delta_{1, j} \exp \left(-p_{0} s_{1}-\zeta_{0}\right)$,

$$
\begin{aligned}
& \partial\left[\cos \left(p_{j} s_{j}+\zeta_{j}\right)-\sin \left(p_{j} s_{j}+\zeta_{j}\right) i_{j}\right] / \partial s_{j}=\partial \exp \left(-\left(p_{j} s_{j}+\zeta_{j}\right) i_{j}\right) / \partial s_{j} \\
& =-p_{j} i_{j} \exp \left(-\left(p_{j} s_{j}+\zeta_{j}\right) i_{j}\right)=-p_{j} \exp \left(-\left(p_{j} s_{j}+\zeta_{j}-\pi / 2\right) i_{j}\right) \\
& =-p_{j}\left[\cos \left(p_{j} s_{j}+\zeta_{j}-\pi / 2\right)-\sin \left(p_{j} s_{j}+\zeta_{j}-\pi / 2\right) i_{j}\right]=-p_{j} s_{e_{j}}\left[\cos \left(p_{j} s_{j}+\zeta_{j}\right)-\sin \left(p_{j} s_{j}+\zeta_{j}\right) i_{j}\right],
\end{aligned}
$$

since $s_{j}$ and $s_{k}$ are real independent variables for each $k \neq j$, where $\delta_{j, k}=0$ for $j \neq k$, while $\delta_{j, j}=1$,

3.1) $S_{e_{j}}\left[\cos \left(p_{j} s_{j}+\zeta_{j}\right)-\sin \left(p_{j} s_{j}+\zeta_{j}\right) i_{j}\right]=-\partial\left[\cos \left(p_{j} s_{j}+\zeta_{j}\right)-\sin \left(p_{j} s_{j}+\zeta_{j}\right) i_{j}\right] / \partial \zeta_{j}$

$$
=\left[\cos \left(p_{j} s_{j}+\zeta_{j}-\pi / 2\right)-\sin \left(p_{j} s_{j}+\zeta_{j}-\pi / 2\right) i_{j}\right]
$$

In the $A_{r}$ Cartesian coordinates we take $t_{j}$ instead of $s_{j}$ in (3.1). If $\phi(z)$ is a differentiable function by $z_{j}$ for each $j, \phi: A_{r} \rightarrow A_{r}, z_{j}=p_{j} t_{j}+\zeta_{j}$, then

3.2) $\partial \exp (-\phi(z)) / \partial\left(q t_{j}\right)=-\left.q[\mathrm{~d} \exp (\xi) / \mathrm{d} \xi]\right|_{\xi=-\phi} \cdot\left(\partial \phi(z) / \partial z_{j}\right) p_{j}$

$$
\begin{aligned}
& =-\left.q p_{j}\left[\sum_{n=1}^{\infty} \sum_{k=1}^{n-1}\left((\xi(z))^{k}\left(\partial \phi(z) / \partial z_{j}\right)\right)(\xi(z))^{n-1-k} / n !\right]\right|_{\xi=-\phi} \\
& =-q p_{j}\left(-\partial \exp (-\phi(z)) / \partial \zeta_{j}\right)=-p_{j} S_{q e_{j}} \exp (-\phi(z)),
\end{aligned}
$$

where either $q=1$ or $q=-1$, since $\partial z_{j} / \partial \zeta_{j}=1$.

That is

3.3) $S_{e_{j}}^{x} \exp \left(-i_{k}\left(\phi_{k}+\zeta_{k}\right)\right)=0$ for each $j \neq k \geq 1$ and any positive number $x>0$,

3.4) $S_{e_{j}}^{x} \exp \left(-i_{j}\left(\phi_{j}+\zeta_{j}\right)\right)=\exp \left(-i_{j}\left(\phi_{j}+\zeta_{j}-x \pi / 2\right)\right)$

3.5) $S_{q e_{j}} e^{-u(p, t ; \zeta)}=e^{-p_{0} s_{1}-\zeta_{0}} T_{j}^{q}\left[i_{0} \delta_{j, 1} \cos \left(p_{1} s_{1}+\zeta_{1}\right)+\left(1-\delta_{j, 1}\right) i_{j-1} \sin \left(p_{1} s_{1}+\zeta_{1}\right) \cdots \cos \left(p_{j} s_{j}+\zeta_{j}\right)\right.$

$$
\left.+\left\{\sum_{k=j}^{2^{r}-2} i_{k} \sin \left(p_{1} s_{1}+\zeta_{1}\right) \cdots \cos \left(p_{k+1} s_{k+1}+\zeta_{k+1}\right)\right\}+i_{2^{r}-1} \sin \left(p_{1} s_{1}+\zeta_{1}\right) \cdots \sin \left(p_{2^{r}-1} s_{2^{r}-1}+\zeta_{2^{r}-1}\right)\right]
$$
$\zeta_{k} \in R$, where $S_{e_{j}}=S_{e_{j}}\left(\zeta_{j}\right)$, the zero power $S_{e_{j}}^{0}=I$ is the unit operator;

$$
S_{-e_{j}}^{x} \exp \left(-i_{j}\left(\phi_{j}+\zeta_{j}\right)\right)=\exp \left(-i_{j}\left(\phi_{j}+\zeta_{j}+x \pi / 2\right)\right)
$$

for each non-negative real number $x \geq 0, \phi_{k}$ and 
in the $A_{r}$ spherical coordinates, where either $q=1$ or $q=-1$ and

3.6) $T_{j}^{x} \xi\left(\zeta_{j}\right):=\xi\left(\zeta_{j}-x \pi / 2\right)$

for any function $\xi\left(\zeta_{j}\right)$ and any real number $x \in R$, where $j \geq 1$. Then in accordance with Formula (3.2) we have:

3.7) $S_{q e_{j}} \exp (-u(p, t ; \zeta))=$

$$
\left.\left[\sum_{n=1}^{\infty} \sum_{k=1}^{n-1}\left((\xi(z))^{k} q i_{j}\right)(\xi(z))^{n-1-k} / n !\right]\right|_{\xi=-u(p, t ; \zeta)}
$$

for $u(p, t ; \zeta)$ given by Formulas $1(8,8.1)$ in the $A_{r}$ Cartesian coordinates, where either $q=1$ or $q=-1$. The integration by parts theorem (Theorem 2 in $\S$ II.2.6 on p. 228 [18]) states: if $a<b$ and two functions $f$ and $g$ are Riemann integrable on the segment $[a, b]$, $F(x)=A+\int_{a}^{x} f(t) \mathrm{d} t$ and $G(x)=B+\int_{a}^{x} g(t) \mathrm{d} t$, where $A$ and $B$ are two real constants, then

$$
\int_{a}^{b} F(x) g(x) \mathrm{d} x=\left.F(x) G(x)\right|_{a} ^{b}-\int_{a}^{b} f(x) G(x) \mathrm{d} x .
$$

Therefore, the integration by parts gives

4) $\int_{0}^{\infty}\left(\partial f(t) / \partial t_{j}\right) \exp (-u(p, t ; \zeta)) \mathrm{d} t_{j}$

$$
\begin{aligned}
= & \left.f(t) \exp (-u(p, t ; \zeta))\right|_{t_{j}=0} ^{t_{j}=\infty} \\
& -\int_{0}^{\infty}\left[f(t)\left(\partial \exp (-u(p, t ; \zeta)) / \partial t_{j}\right)\right] \mathrm{d} t_{j} .
\end{aligned}
$$

Using the change of variables $t \mapsto s$ with the unit Jacobian $\partial\left(t_{1}, \cdots, t_{n}\right) / \partial\left(s_{1}, \cdots, s_{n}\right)$ and applying the Fubini's theorem componentwise to $f_{j} i_{j}$ we infer:

$$
\text { 5) } \begin{aligned}
& \int_{U_{1}, \cdots, 1}\left(\partial f(t) / \partial t_{j}\right) \exp (-u(p, t ; \zeta)) \mathrm{d} t=\int_{s_{1} \geq s_{2} \geq \cdots \geq s_{n} \geq 0}\left(\partial f(t) / \partial t_{j}\right) \exp (-u(p, t ; \zeta)) \mathrm{d} s \\
& =\int_{0}^{\infty} \cdots \int_{0}^{\infty}\left[\int_{s_{j+1}}^{\infty}\left(\partial f(t) / \partial t_{j}\right) \exp (-u(p, t ; \zeta)) \mathrm{d} s_{j}\right] \mathrm{d} t^{j} \\
& =-\left[\int_{0}^{\infty} \cdots \int_{0}^{\infty} f\left(t^{j}\right) \exp \left(-u\left(p, t^{j} ; \zeta\right)\right) \mathrm{d} t^{j}\right]+\left[p_{0}+\sum_{k=1}^{j} p_{k} S_{e_{k}}\right] \int_{0}^{\infty} \cdots \int_{0}^{\infty} f(t) \exp (-u(p, t ; \zeta)) \mathrm{d} t
\end{aligned}
$$

in the $A_{r}$ spherical coordinates, or

$$
\text { 5.1) } \begin{aligned}
& \int_{U_{1}, \cdots, 1}\left(\partial f(t) / \partial t_{j}\right) \exp (-u(p, t ; \zeta)) \mathrm{d} t \\
& =-\left[\int_{0}^{\infty} \cdots \int_{0}^{\infty} f\left(t^{j}\right) \exp \left(-u\left(p, t^{j} ; \zeta\right)\right) \mathrm{d} t^{j}\right]+\left[p_{0}+p_{j} S_{e_{j}}\right] \int_{0}^{\infty} \cdots \int_{0}^{\infty} f(t) \exp (-u(p, t ; \zeta)) \mathrm{d} t
\end{aligned}
$$

in the $A_{r}$ Cartesian coordinates, since $\partial \exp \left(-\left(p_{0} s_{1}+\zeta_{0}\right)\right) / \partial t_{j}=-p_{0} \exp \left(-\left(p_{0} s_{1}+\zeta_{0}\right)\right)$ for each $1 \leq j \leq n$. This gives Formula (1), where

6) $F^{n-1 ; t^{j}}\left(f\left(t^{j}\right) \chi_{U_{1, \cdots, 1}}, u\left(p, t^{j} ; \zeta\right) ; p ; \zeta\right)=\int_{0}^{\infty} \cdots \int_{0}^{\infty} f\left(t^{j}\right) \exp \left(-u\left(p, t^{j} ; \zeta\right)\right) \mathrm{d} t^{j}$

$$
=\int_{0}^{\infty} \mathrm{d} t_{1} \cdots \int_{0}^{\infty} \mathrm{d} t_{j-1} \int_{0}^{\infty} d t_{j+1} \cdots \int_{0}^{\infty}\left(\mathrm{d} t_{n}\right) f\left(t^{j}\right) \exp \left(-u\left(p, t^{j} ; \zeta\right)\right)
$$

is the non-commutative transform by

$$
t^{j}=\left(t_{1}, \cdots, t_{j-1}, 0, t_{j+1}, \cdots, t_{n}\right) \text {. }
$$

\section{Remark}

Shift operators of the form $\xi(x+\phi)=\exp (\phi d / d x) \xi(x)$ in real variables are also frequently used in the class of infinite differentiable functions with converging Taylor series expansion in the corresponding domain.

It is possible to use also the following convention. One can put

$$
\begin{aligned}
& \cos \left(\phi_{1}+\zeta_{1}\right)=\cos \left(\phi_{1}+\zeta_{1}\right) \cos \left(\psi_{2}\right) \cdots \cos \left(\psi_{2^{r}-1}\right), \cdots, \\
& \sin \left(\phi_{1}+\zeta_{1}\right) \cdots \cos \left(\phi_{k}+\zeta_{k}\right) \\
& =\sin \left(\phi_{1}+\zeta_{1}\right) \cdots \cos \left(\phi_{k}+\zeta_{k}\right) \cos \left(\psi_{k+1}\right) \cdots \cos \left(\psi_{2^{r}-1}\right),
\end{aligned}
$$

where $\psi_{j}=0$ for each $j \geq 1,2 \leq k<2^{r}-1$, so that $T_{j}^{l} \cos \left(\phi_{1}+\zeta_{1}\right)=0$ for each $j>1$ and $l \geq 1$,
$T_{j}^{l} \sin \left(\phi_{1}+\zeta_{1}\right) \cdots \cos \left(\phi_{k}+\zeta_{k}\right)=0$ for each $j>k$ and $l \geq 1$, where $T_{j}^{l} \xi=T_{j}^{l-1}\left(T_{j} \xi\right)$ is the iterated composition for $l>1, l \in N$. Then $T_{j}^{l} e^{-u(p, t ; \zeta)}$ gives with such convention the same result as $S_{e_{j}}^{l} e^{-u(p, t ; \zeta)}$, so one can use the symbolic notation $T_{j}^{l} e^{-u(p, t ; \zeta)}=e^{-u\left(p, t ; \zeta-i_{j} \pi l / 2\right)}$. But to avoid misunderstanding we shall use $S_{e_{j}}$ and $T_{j}$ in the sense of Formulas 12(3.1-3.7).

It is worth to mention that instead of 12(3.7) also the formulas

1) $\exp \left(p_{1} i_{1}+\cdots+p_{n} i_{n}\right)=\cos (\phi)+M \sin (\phi)$ with $\phi:=\phi(p):=\left[p_{1}^{2}+\cdots+p_{n}^{2}\right]^{1 / 2}$ and $M=\left(p_{1} i_{1}+\cdots+p_{n} i_{n}\right) / \phi$ for $\phi \neq 0, e^{0}=1$;

2) $\partial \exp \left(p_{1} i_{1}+\cdots+p_{n} i_{n}\right) / \partial p_{j}$

$$
\begin{aligned}
= & {[-\sin (\phi)+M \cos (\phi)] p_{j} / \phi } \\
& +\left(\phi i_{j}-M p_{j}\right) \phi^{-2} \sin (\phi)
\end{aligned}
$$


and $\partial\left(p_{j} t_{j}+\zeta_{j}\right) / \partial \zeta_{j}=1$ can be used.

\subsection{Theorem}

Let $f(t)$ be a function-original. Suppose that $u(p, t ; \zeta)$ is given by $2(1,2,2.1)$ or $1(8,8.1)$ over the Cayley-Dickson algebra $A_{r}$ with $2 \leq r<\infty$. Then a (super) derivative of an image is given by the following formula:

1) $\left(\partial F^{n}(f(t), u ; p ; \zeta) / \partial p\right) \cdot h=-F^{n}\left(f(t) s_{1}, u ; p ; \zeta\right) h_{0}-S_{e_{1}} F^{n}\left(f(t) s_{1}, u ; p ; \zeta\right) h_{1}-\cdots-S_{e_{n}} F^{n}\left(f(t) s_{n}, u ; p ; \zeta\right) h_{n}$

in the $A_{r}$ spherical coordinates, or

1.1) $\left(\partial F^{n}(f(t), u ; p ; \zeta) / \partial p\right) \cdot h=-F^{n}\left(\left(f(t) s_{1}, u ; p ; \zeta\right)\right) h_{0}-S_{e_{1}} F^{n}\left(f(t) t_{1}, u ; p ; \zeta\right) h_{1}-\cdots-S_{e_{n}} F^{n}\left(f(t) t_{n}, u ; p ; \zeta\right) h_{n}$

in the $A_{r}$ Cartesian coordinates for each

$h=h_{0} i_{0}+\cdots+h_{n} i_{n} \in A_{r}$, where $h_{0}, \cdots, h_{n} \in R$,

$2^{r-1} \leq n \leq 2^{r}-1, \quad p \in W_{f}$.

Proof. The inequalities $a_{1}(f)<\operatorname{Re}(p)<a_{-1}(f)$ are equivalent to the inequalities

$a_{1}(f(t)|t|)<\operatorname{Re}(p)<a_{-1}(f(t)|t|)$, since $\lim _{|t| \rightarrow+\infty} \exp (-b|t|)|t|=0$ for each $b>0$. An image
$F^{n}(f(t), u ; p ; \zeta)$ is a holomorphic function by $p$ for $a_{1}(f)<\operatorname{Re}(p)<a_{-1}(f)$ by Theorem 4 , also $\left|\int_{0}^{\infty} e^{-c t} t^{n} \mathrm{~d} t\right|<\infty$ for each $c>0$ and $n=0,1,2, \cdots$.

Thus it is possible to differentiate under the sign of the integral:

2) $\left(\partial\left(\int_{R^{n}} f(t) \exp (-u(p, t ; \zeta)) d t\right) / \partial p\right) \cdot h=\sum_{v \in\{-1,1\}^{n}}\left(\partial\left(\int_{U_{v}} f(t) \exp (-u(p, t ; \zeta)) \chi_{U_{v}} \mathrm{~d} t\right) / \partial p\right) \cdot h$

$$
=\int_{R^{n}} f(t)(\partial \exp (-u(p, t ; \zeta)) / \partial p) \cdot h \mathrm{~d} t .
$$

Due to Formulas 12(3,3.2) we get:

3) $(\partial \exp (-u(p, t ; \zeta)) / \partial p) \cdot h=-\exp (-u(p, t ; \zeta)) s_{1} h_{0}-S_{e_{1}} \exp (-u(p, t ; \zeta)) s_{1} h_{1}-\cdots-S_{e_{n}} \exp (-u(p, t ; \zeta)) s_{n} h_{n}$

in the $A_{r}$ spherical coordinates, or

4) $(\partial \exp (-u(p, t ; \zeta)) / \partial p) \cdot h=-\exp (-u(p, t ; \zeta)) s_{1} h_{0}-S_{e_{1}} \exp (-u(p, t ; \zeta)) t_{1} h_{1}-\cdots-S_{e_{n}} \exp (-u(p, t ; \zeta)) t_{n} h_{n}$

in the $A_{r}$ Cartesian coordinates.

Thus from Formulas $(2,3)$ we deduce Formula (1).

\subsection{Theorem}

If $f(t)$ is a function-original, then

1) $F^{n}(f(t-\tau), u ; p ; \zeta)=F^{n}(f(t), u ; p ; \zeta+\langle p, \tau])$ for either

i) $u(p, t ; \zeta)=p_{0} s_{1}+M(p, t ; \zeta)+\zeta_{0}$ or

3) $F^{n}\left(\left(f \chi_{U_{1, \ldots, 1}}\right)(t-\tau), u ; p ; \zeta\right)=\int_{\tau_{1}}^{\infty} \cdots \int_{\tau_{n}}^{\infty} f(t-\tau) e^{-u(p, t ; \zeta)} \mathrm{d} t$

$$
=\int_{U_{1, \ldots, 1}} f(t) e^{-u(p, \xi ; \zeta+\langle p, \tau])} \mathrm{d} \xi=F^{n}\left(\left(f \chi_{U_{1, \cdots, 1}}\right)(t), u ; p ; \zeta+\langle p, \tau]\right),
$$

due to Formulas $1(7,8)$ and $2(1,2,2.1,4)$, since

$p_{0} s_{1}(n ; t)+\zeta_{0}=p_{0} s_{1}(n ; \xi)+\zeta_{0}+p_{0} s_{1}(n ; \tau)$ and

$p_{j} t_{j}+\zeta_{j}=p_{j} \xi_{j}+\left(\zeta_{j}+p_{j} \tau_{j}\right)$ and

$p_{j} s_{j}(n ; t)+\zeta_{j}=p_{j} s_{j}(n ; \xi)+\left(\zeta_{j}+p_{j} s_{j}(n ; \tau)\right)$ for each

$j=1, \cdots, 2^{r}-1$, where $t=\xi+\tau$. Symmetrically we get

(2) for $U_{v}$ instead of $U_{1, \cdots, 1}$. Naturally, that the multiparameter non-commutative Laplace integral for an original $f$ can be considered as the sum of $2^{n}$ in- ii) $u(p, t ; \zeta)=\langle p, t)+\zeta$ over $A_{r}$ with $2 \leq r<\infty$ in a domain $p \in W_{f}$, where $\tau \in R^{n}, 2^{r-1} \leq n \leq 2^{r}-1$,

2) $\langle p, \tau]=p_{0} s_{1}+p_{1} s_{1} i_{1}+\cdots+p_{n} s_{n} i_{n}$ with $s_{j}=s_{j}(n ; \tau)$ for each $j$ in the first (i) and $\langle p, \tau]=\langle p, \tau)$ in the second (ii) case (see also Formulas $1(8), 2(1,2,2.1))$.

Proof. For $p$ in the domain $\operatorname{Re}(p)>a_{1}$ the identities are satisfied: tegrals by the sub-domains $U_{v}$ :

4) $\int_{R^{n}} f(t) \exp (-u(p, t ; \zeta)) \mathrm{d} t$

$$
=\sum_{v \in\{-1,1\}^{n}} \int_{R^{n}} f(t) \exp (-u(p, t ; \zeta)) \chi_{U_{v}}(t) \mathrm{d} t .
$$

The summation by all possible $v \in\{-1,1\}^{n}$ gives Formula (1).

\section{Copyright}




\subsection{Note}

In view of the definition of the non-commutative transform $F^{n}$ and $u(p, t ; \zeta)$ and Theorem 14 the term $\zeta_{1} i_{1}+\cdots+\zeta_{2} i_{1}{ }^{r}$ has the natural interpretation as the initial phase of a retardation.

\subsection{Theorem}

If $f(t)$ is a function-original with values in $A_{r}$ for
$2 \leq r<\infty, 2^{r-1} \leq n \leq 2^{r}-1, \quad b \in R$, then

1) $F^{n}\left(e^{b\left(t_{1}+\cdots+t_{n}\right)} f(t), u ; p ; \zeta\right)=F^{n}(f(t), u ; p-b ; \zeta)$

for each $a_{-1}+b>\operatorname{Re}(p)>a_{1}+b$, where $u$ is given by $1(8,8.1)$ or $2(1,2)$.

Proof. In accordance with Expressions 1(8,8.1) and $2(1,2,2.1)$ one has

$u(p, t ; \zeta)-b\left(t_{1}+\cdots+t_{n}\right)=u(p-b, t ; \zeta)$. If $a_{-1}+b>\operatorname{Re}(p)>a_{1}+b$, then the integral

2) $F^{n}\left(e^{b\left(t_{1}+\ldots+t_{n}\right)} f(t) \chi_{U_{v}}(t), u ; p ; \zeta\right)=\int_{U_{v}} f(t) e^{b\left(t_{1}+\ldots+t_{n}\right)} \exp (-u(p, t ; \zeta)) \mathrm{d} t$ $=\int_{U_{v}} f(t) \exp (-u(p-b, t ; \zeta)) \mathrm{d} t=F^{n}\left(f(t) \chi_{U_{v}}(t), u ; p-b ; \zeta\right)$

converges. Applying Decomposition 14(4) we deduce Formula (1).

\subsection{Theorem}

Let a function $f(t)$ be a real valued original, $F(p ; \zeta)=F^{n}(f(t) ; u ; p ; \zeta)$, where the function $u(p, t ; \zeta)$ is given by $1(8,8.1)$ or $2(1,2,2.1)$. Let also $G(p ; \zeta)$ and $q(p)$ be locally analytic functions such that

1) $F^{n}(g(t, \tau) ; u ; p ; \zeta)=G(p ; \zeta) \exp (-u(q(p), \tau ; \zeta))$ for $u=\langle p, t)+\zeta$ or $u=p_{0}\left(t_{1}+\cdots+t_{n}\right)+M(p, t ; \zeta)+\zeta_{0}$, then

2) $F^{n}\left(\int_{R^{n}} g(t, \tau) f(\tau) \mathrm{d} \tau ; u ; p ; \zeta\right)$

$$
=G(p ; \zeta) F(q(p) ; \zeta)
$$

for each $p \in W_{g}$ and $q(p) \in W_{f}$, where $2 \leq r<\infty$, $2^{r-1} \leq n \leq 2^{r}-1$.

Proof. If $p \in W_{g}$ and $q(p) \in W_{f}$, then in view of the Fubini's theorem and the theorem conditions a change of an integration order gives the equalities:

$$
\begin{aligned}
& \int_{R^{n}}\left(\int_{R^{n}} g(t, \tau) f(\tau) \mathrm{d} \tau\right) \exp (-u(p, t ; \zeta)) \mathrm{d} t \\
& =\int_{R^{n}}\left(\int_{R^{n}} g(t, \tau) \exp (-u(p, t ; \zeta)) \mathrm{d} t\right) f(\tau) \mathrm{d} \tau \\
& =\int_{R^{n}} G(p ; \zeta) \exp (-u(q(p), \tau ; \zeta)) f(\tau) \mathrm{d} \tau \\
& =G(p ; \zeta) \int_{R^{n}} f(\tau) \exp (-u(q(p), \tau ; \zeta) \mathrm{d} \tau \\
& =G(p ; \zeta) F(q(p) ; \zeta),
\end{aligned}
$$

since $t, \tau \in R^{n}$ and the center of the algebra $A_{r}$ is $R$.

\subsection{Theorem}

If a function $f(t) \chi_{U_{1, \cdots, 1}}$ is original together with its derivative $\partial^{n} f(t) \chi_{U_{1, \cdots, 1}}(t) / \partial s_{1} \cdots \partial s_{n}$ or $\partial^{n} f(t) \chi_{U_{1}, \ldots, 1}(t) / \partial t_{1} \cdots \partial t_{n}$, where $F_{u}^{n}(p ; \zeta)$ is an image function of $f(t) \chi_{U_{1} \ldots 1}$ over the Cayley-Dickson algebra $A_{r}$ with $2 \leq r \in \dddot{N}^{1}, 2^{r-1} \leq n \leq 2^{r}-1$, for $u=p_{0} s_{1}+M(p, t ; \zeta)+\zeta_{0}$ given by $2(1,2,2.1)$, then

1) $\lim _{p \rightarrow \infty}\left\{\left[p_{0}+p_{1} S_{e_{1}}\right] p_{2} S_{e_{2}} \cdots p_{n} S_{e_{n}} F_{u}^{n}(p ; \zeta)+\sum_{m=0}^{n-1}(-1)^{m}\right.$

$$
\left.\sum_{1 \leq j_{1}<\cdots<j_{n-m} \leq n ; 1 \leq l_{1}<\cdots<l_{m} \leq n ; l_{\alpha} \neq j_{\beta} \forall \alpha, \beta}\left[p_{0} \delta_{1, j_{1}}+p_{j_{1}} S_{e_{j_{1}}}\right] p_{j_{2}} S_{e_{j_{2}}} \cdots p_{j_{n-m}} S_{e_{j_{n-m}}} F_{u}^{n-m}\left(p^{(l)} ; \zeta\right)\right\}=(-1)^{n+1} f(0) e^{-u(0,0 ; \zeta)},
$$

or

$$
\text { 1.1) } \begin{aligned}
& \lim _{p \rightarrow \infty}\left\{\left[p_{0}+p_{1} S_{e_{1}}\right]\left[p_{0}+p_{2} S_{e_{2}}\right] \cdots\left[p_{0}+p_{n} S_{e_{n}}\right] F_{u}^{n}(p ; \zeta)+\sum_{m=0}^{n-1}(-1)^{m}\right. \\
& \left.\sum_{1 \leq j_{1}<\cdots<j_{n-m} \leq n ; 1 \leq l_{1}<\cdots<l_{m} \leq n ; l_{\alpha} \neq j_{\beta} \forall \alpha, \beta}\left[p_{0}+p_{j_{1}} S_{e_{j_{1}}}\right]\left[p_{0}+p_{j_{2}} S_{e_{j_{2}}}\right] \cdots\left[p_{0}+p_{j_{n-m}} S_{e_{j_{n-m}}}\right] F_{u}^{n-m}\left(p^{(l)} ; \zeta\right)\right\} \\
= & (-1)^{n+1} f(0) e^{-u(0,0 ; \zeta)}
\end{aligned}
$$

for $u(p, t ; \zeta)$ given by $1(8,8.1)$, where

$$
f(0)=\lim _{t \in U_{1, \cdots, 1} ; t \rightarrow 0} f(t),
$$

$p$ tends to the infinity inside the angle

$$
|\operatorname{Arg}(p)|<\pi / 2-\delta
$$

for some $0<\delta<\pi / 2,1 \leq j \leq 2^{r}-1$, $p^{(l)}=\sum_{j=0, j \notin(l)}^{n} p_{j} i_{j}, \quad(l)=\left(l_{1}, \cdots, l_{m}\right)$. If the restriction 


$$
\left.f(t)\right|_{t_{j_{1}}=0, \cdots, t_{j_{m}}=0 ; t_{k}=\infty \forall k \notin\left\{j_{1}, \cdots, j_{m}\right\}}=\lim _{t \in U_{1}, \cdots, 1 ; t_{j_{1}} \rightarrow 0, \cdots, t_{j_{m}} \rightarrow 0 ; t_{k} \rightarrow \infty \forall k \notin\left\{j_{1}, \cdots, j_{m}\right\}} f(t)
$$

exists for all $1 \leq j_{1}<\cdots<j_{m} \leq n$, then

$$
\text { 2) } \begin{aligned}
& \lim _{p \rightarrow 0}\left\{\left[p_{0}+p_{1} S_{e_{1}}\right] p_{2} S_{e_{2}} \cdots p_{n} S_{e_{n}} F_{u}^{n}(p ; \zeta)+\sum_{m=0}^{n-1}(-1)^{m}\right. \\
& \left.\sum_{1 \leq j_{1}<\cdots<j_{n-m} \leq n ; 1 \leq l_{1}<\cdots<l_{m} \leq n ; l_{\alpha} \neq j_{\beta} \forall \alpha, \beta}\left[p_{0} \delta_{1, j_{1}}+p_{j_{1}} S_{e_{j_{1}}}\right] p_{j_{2}} S_{e_{j_{2}}} \cdots p_{j_{n-m}} S_{e_{j_{n-m}}} F_{u}^{n-m}\left(p^{(l)} ; \zeta\right)\right\} \\
= & \left.\sum_{m=0}^{n-1}(-1)^{m} \sum_{1 \leq j_{1}<\ldots<j_{m} \leq n} f(t)\right|_{t_{j_{1}}=0, \cdots, t_{j_{m}}=0 ; t_{k}=\infty \forall k \notin\left\{j_{1}, \cdots, j_{m}\right\}} e^{-u(0,0, \zeta)}
\end{aligned}
$$

in the $A_{r}$ spherical coordinates or

$$
\text { 2.1) } \begin{aligned}
& \lim _{p \rightarrow 0}\left\{\left[p_{0}+p_{1} S_{e_{1}}\right]\left[p_{0}+p_{2} S_{e_{2}}\right] \cdots\left[p_{0}+p_{n} S_{e_{n}}\right] F_{u}^{n}(p ; \zeta)+\sum_{m=0}^{n-1}(-1)^{m}\right. \\
& \left.\sum_{1 \leq j_{1}<\cdots<j_{n-m} \leq n ; 1 \leq 1_{1}<\cdots<l_{m} \leq n ; l_{\alpha} \neq j_{\beta} \forall \alpha, \beta}\left[p_{0}+p_{j_{1}} S_{e_{j_{1}}}\right]\left[p_{0}+p_{j_{2}} S_{e_{j_{2}}}\right] \cdots\left[p_{0}+p_{j_{n-m}} S_{e_{j_{n-m}}}\right] F_{u}^{n-m}\left(p^{(l)} ; \zeta\right)\right\} \\
= & \left.\sum_{m=0}^{n-1}(-1)^{m} \sum_{1 \leq j_{1}<\ldots<j_{m} \leq n} f(t)\right|_{t_{j_{1}}=0, \cdots, t_{j_{m}}=0 ; t_{k}=\infty \forall k \notin\left\{j_{1}, \cdots, j_{m}\right\}} e^{-u(0,0, \zeta)}
\end{aligned}
$$

in the $A_{r}$ Cartesian coordinates, where $p \rightarrow 0$ inside the same angle.

Proof. In accordance with Theorem 12 the equality follows:

3) $F^{n}\left(\left(\partial f(t) / \partial s_{j}\right) \chi_{U_{1, \cdots, 1}}(t), u ; p ; \zeta\right)=\left[p_{0} \delta_{1, j}+p_{j} S_{e_{j}}\right] F^{n}\left(f(t) \chi_{U_{1, \cdots, 1}}(t), u(p, t ; \zeta), p ; \zeta\right)$

$$
-F^{n-1 ; t^{j}}\left(f\left(t^{j}\right) \chi_{U_{1, \cdots, 1}}, u\left(p, t^{j} ; \zeta\right) ; p ; \zeta\right)
$$

for $u=u(p, t ; \zeta)=p_{0} s_{1}+M(p, t ; \zeta)+\zeta_{0}$ in the $A_{r}$ spherical coordinates, or

3.1) $F^{n}\left(\left(\partial f(t) / \partial t_{j}\right) \chi_{U_{1, \cdots, 1}}(t), u ; p ; \zeta\right)=\left[p_{0}+p_{j} S_{e_{j}}\right] F^{n}\left(f(t) \chi_{U_{1, \cdots, 1}}(t), u(p, t ; \zeta), p ; \zeta\right)$

$$
-F^{n-1 ; t^{j}}\left(f\left(t^{j}\right) \chi_{U_{1, \cdots, 1}}, u\left(p, t^{j} ; \zeta\right) ; p ; \zeta\right)
$$

in the $A_{r}$ Cartesian coordinates, since

3.2) $\partial f(t(s)) / \partial s_{j}=-\partial f(t) / \partial t_{j-1}+\partial f(t) / \partial t_{j}$

for each $j \geq 2, \partial f(t(s)) / \partial s_{1}=\partial f(t) / \partial t_{1}$,

where $p=p_{0}+p_{1} i_{1}+\cdots+p_{2^{r}-1} i_{2^{r}-1} \in A_{r}$,

$p_{0}, \cdots, p_{2^{r}-1} \in R,\left\{i_{0}, \cdots, i_{2^{r}-1}\right\}^{r}$ are the generators of the

Cayley-Dickson algebra $A_{r}, s_{n+l}=0$ for each $l \geq 1$, the zero power $S_{e_{j}}^{0}=I$ is the unit operator. For short we write $f$ instead of $f \chi_{U_{1, \ldots 1}}$. Thus the limit exists:

4) $F^{n-1 ; t^{j}}\left(f\left(t^{j}\right), u\left(p, t^{j} ; \zeta\right) ; p ; \zeta\right)=$

$$
\begin{aligned}
& \lim _{t_{j} \rightarrow+0} \int_{0}^{\infty} \mathrm{d} t_{1} \cdots \int_{0}^{\infty} \mathrm{d} t_{j-1} \int_{0}^{\infty} \mathrm{d} t_{j+1} \cdots \int_{0}^{\infty}\left(\mathrm{d} t_{n}\right) \\
& f(t) \exp (-u(p, t ; \zeta)) .
\end{aligned}
$$

Mention, that

$$
\left(\cdots\left(\left(t^{1}\right)^{2}\right) \cdots\right)^{j}=\left(0, \cdots, 0, t_{j}, \cdots, t_{n}: t_{j}=0\right)
$$

for every $1 \leq j \leq n$, since $t_{k}=s_{k}-s_{k+1}$ for each $1 \leq k \leq n$. We apply these Formulas $(3,4)$ by induction $j=1, \cdots, n, \quad 2^{r-1} \leq n \leq 2^{r}-1$, to $\partial^{n} f(t) / \partial s_{1} \cdots \partial s_{n}$, $\cdots, \partial^{n-j+1} f(t) / \partial s_{j} \cdots \partial s_{n}, \cdots, \partial f(t) / \partial s_{n}$ instead of $\partial f(t) / \partial s_{j}$.

From Note 8 [4] it follows, that in the $A_{r}$ spherical coordinates

$$
\lim _{p \rightarrow \infty,|\operatorname{Arg}(p)|<\pi / 2-\delta} F^{n}\left(\left(\partial^{n} f(t) / \partial s_{1} \cdots \partial s_{n}\right) \chi_{U_{1, \cdots, 1}}, u ; p ; \zeta\right)
$$

$=0$,

also in the $A_{r}$ Cartesian coordinates

$$
\begin{aligned}
& \left.\lim _{p \rightarrow \infty, \mid A r g(p)<\pi / 2-\delta} F^{n}\left(\left(\partial^{n} f(t) / \partial t_{1} \cdots \partial t_{n}\right)\right) \chi_{U_{1, \cdots, 1}}, u ; p ; \zeta\right) \\
& =0,
\end{aligned}
$$

which gives the first statement of this theorem, since $u(p, 0, \zeta)=u(0, t ; \zeta)=u(0,0, \zeta)$ and 
$F_{u}^{0}\left(p^{(1, \cdots, 1)} ; \zeta\right)=f(0) e^{-u(0,0, \zeta)}$, while $F_{u}^{n}(p ; \zeta)$ is defined for each $\operatorname{Re}(p)>0$.
If the limit $f\left(t^{\langle j\rangle}\right)$ exists, where $t^{\langle j\rangle}:=\left(t_{1}, \cdots, t_{j}, \cdots, t_{n}: t_{j}=\infty\right)$, then

5) $\lim _{t_{j} \rightarrow \infty} \int_{0}^{\infty} \mathrm{d} t_{1} \cdots \int_{0}^{\infty} \mathrm{d} t_{j-1} \int_{0}^{\infty} \mathrm{d} t_{j+1} \cdots \int_{0}^{\infty}\left(\mathrm{d} t_{n}\right) f(t) \exp (-u(p, t ; \zeta))=: F^{n-1 ;\left\langle t^{j}\right\rangle}\left(f\left(t^{\langle j\rangle}\right), u\left(p, t^{\langle j\rangle} ; \zeta\right) ; p ; \zeta\right)$.

Certainly, $\left(\cdots\left(\left(t^{\langle 1\rangle}\right)^{\langle 2\rangle}\right) \cdots\right)^{\langle j\rangle}=\left(t_{1}, \cdots, t_{n}: t_{1}=\infty, \cdots, t_{j}=\infty\right)$ for each $1 \leq j \leq n$. Therefore, the limit exists:

$$
\begin{aligned}
& \lim _{p \rightarrow 0,|A r g(p)|<\pi / 2-\delta} \int_{U_{1, \cdots, 1}}\left(\partial^{n} f(t) / \partial s_{1} \cdots \partial s_{n}\right) \exp \left(-p_{0} s_{1}-\zeta_{0}-M(p, t ; \zeta)\right) \\
= & \int_{U_{1, \ldots, 1}}\left(\partial^{n} f(t) / \partial s_{1} \cdots \partial s_{n}\right) e^{-u(0,0 ; \zeta)} \mathrm{d} t=\left.\sum_{m=0}^{n}(-1)^{m} \sum_{1 \leq j_{1}<\cdots<j_{m} \leq n} f(t)\right|_{t_{j_{1}}=0, \cdots, t_{j_{m}}=0 ; t_{k}=\infty \forall k \notin\left\{j_{1}, \cdots, j_{m}\right\}} \\
= & \lim _{p \rightarrow 0,|A r g(p)|<\pi / 2-\delta}\left\{\left[p_{0}+p_{1} S_{e_{1}}\right] p_{2} S_{e_{2}} \cdots p_{n} S_{e_{n}} F_{u}^{n}(p ; \zeta)+\sum_{m=0}^{n-1}(-1)^{m}\right. \\
& \left.\sum_{1 \leq j_{1}<\cdots<j_{n-m} \leq n ; 1 \leq l_{1}<\cdots<l_{m} \leq n ; l_{\alpha} \neq j_{\beta} \forall \alpha, \beta}\left[p_{0} \delta_{1, j_{1}}+p_{j_{1}} S_{e_{j_{1}}}\right] p_{j_{2}} S_{e_{j_{2}}} \cdots p_{j_{n-m}} S_{e_{j_{n-m}}} F_{u}^{n-m}\left(p^{(l)} ; \zeta\right)+(-1)^{n} f(0) e^{-u(0,0, \zeta)}\right\},
\end{aligned}
$$

from which the second statement of this theorem follows in the $A_{r}$ spherical coordinates and analogously in the $A_{r}$ Cartesian coordinates using Formula (3.1).

\subsection{Definitions}

Let $X$ and $Y$ be two $R$ linear normed spaces which are also left and right $A_{r}$ modules, where $1 \leq r$. Let $Y$ be complete relative to its norm. We put $X^{\otimes k}:=X \otimes_{R} \cdots \otimes_{R} X$ is the $k$ times ordered tensor product over $R$ of $X$. By $L_{q, k}\left(X^{\otimes k}, Y\right)$ we denote a family of all continuous $k$ times $R$ poly-linear and $A_{r}$ additive operators from $X^{\otimes k}$ into $Y$. Then $L_{q, k}\left(X^{\otimes k}, Y\right)$ is also a normed $R$ linear and left and right $A_{r}$ module complete relative to its norm. In particular, $L_{q, 1}(X, Y)$ is denoted also by $L_{q}(X, Y)$. We present $X$ as the direct sum

$X=X_{0} i_{0} \oplus \cdots \oplus X_{2^{r}-12^{r}-1}$, where $X_{0}, \cdots, X_{2^{r}-1}$ are pairwise isomorphic real normed spaces. If

$A \in L_{q}(X, Y)$ and $A(x b)=(A x) b$ or $A(b x)=b(A x)$ for each $x \in X_{0}$ and $b \in A_{r}$, then an operator $A$ we call right or left $A_{r}$-linear respectively. An $R$ linear space of left (or right) $k$ times $A_{r}$ poly-linear operators is denoted by $L_{l, k}\left(X^{\otimes k}, Y\right)$ (or $L_{r, k}\left(X^{\otimes k}, Y\right.$ ) respectively).

We consider a space of test function $D:=D\left(R^{n}, Y\right)$ consisting of all infinite differentiable functions

$f: R^{n} \rightarrow Y$ on $R^{n}$ with compact supports. A sequence of functions $f_{n} \in D$ tends to zero, if all $f_{n}$ are zero outside some compact subset $K$ in the Euclidean space $R^{n}$, while on it for each $k=0,1,2, \cdots$ the sequence $\left\{f_{n}^{(k)}: n \in N\right\}$ converges to zero uniformly. Here as usually $f^{(k)}(t)$ denotes the $k$-th derivative of $f$, which is a $k$ times $R$ poly-linear symmetric operator from $\left(R^{n}\right)^{\otimes k}$ to $Y$, that is

$$
f^{(k)}(t) \cdot\left(h_{1}, \cdots, h_{k}\right)=f^{(k)}(t) \cdot\left(h_{\sigma(1)}, \cdots, h_{\sigma(k)}\right) \in Y
$$

for each $h_{1}, \cdots, h_{k} \in R^{n}$ and every transposition $\sigma:\{1, \cdots, k\} \rightarrow\{1, \cdots, k\}, \sigma$ is an element of the symmetric group $S_{k}, t \in R^{n}$. For convenience one puts $f^{(0)}=f$. In particular,

$$
f^{(k)}(t) \cdot\left(e_{j_{1}}, \cdots, e_{j_{k}}\right)=\partial^{k} f(t) / \partial t_{j_{1}} \cdots \partial t_{j_{k}}
$$

for all $1 \leq j_{1}, \cdots, j_{k} \leq n$, where $e_{j}=(0, \cdots, 0,1,0, \cdots, 0) \in R^{n}$ with 1 on the $j$-th place.

Such convergence in $D$ defines closed subsets in this space $D$, their complements by the definition are open, that gives the topology on $D$. The space $D$ is $R$ linear and right and left $A_{r}$ module.

By a generalized function of class $D^{\prime}:=\left[D\left(R^{n}, Y\right)\right]^{\prime}$ is called a continuous $R$-linear $A_{r}$-additive function $g: D \rightarrow A_{r}$. The set of all such functionals is denoted by $D^{\prime}$. That is, $g$ is continuous, if for each sequence $f_{n} \in D$, converging to zero, a sequence of numbers $g\left(f_{n}\right)=:\left[g, f_{n}\right) \in A_{r}$ converges to zero for $n$ tending to the infinity.

A generalized function $g$ is zero on an open subset $V$ in $R^{n}$, if $[g, f)=0$ for each $f \in D$ equal to zero outside $V$. By a support of a generalized function $g$ is called the family, denoted by $\operatorname{supp}(g)$, of all points $t \in R^{n}$ such that in each neighborhood of each point $t \in \operatorname{supp}(g)$ the functional $g$ is different from zero. The addition of generalized functions $g, h$ is given by the formula:

1) $[g+h, f):=[g, f)+[h, f)$.

The multiplication $g \in D^{\prime}$ on an infinite differentiable function $w$ is given by the equality:

2) $[g w, f)=[g, w f)$ either for $w: R^{n} \rightarrow A_{r}$ and each test function $f \in D$ with a real image $f\left(R^{n}\right) \subset R$, where $R$ is embedded into $Y$; or 
$w: R^{n} \rightarrow R$ and $f: R^{n} \rightarrow Y$.

A generalized function $g^{\prime}$ prescribed by the equation:

3) $\left[g^{\prime}, f\right):=-\left[g, f^{\prime}\right)$ is called a derivative $g^{\prime}$ of a generalized function $g$, where $f^{\prime} \in D\left(R^{n}, L_{q}\left(R^{n}, Y\right)\right)$, $g^{\prime} \in\left[D\left(R^{n}, L_{q}\left(R^{n}, Y\right)\right)\right]^{\prime}$.

Another space $B:=B\left(R^{n}, Y\right)$ of test functions consists of all infinite differentiable functions $f: R^{n} \rightarrow Y$ such that the limit $\lim _{t \rightarrow \rightarrow+\infty}|t|^{m} f^{(j)}(t)=0$ exists for each $m=0,1,2, \cdots, j=0,1,2, \cdots$. A sequence $f_{n} \in B$ is called converging to zero, if the sequence $|t|^{m} f_{n}^{(j)}(t)$ converges to zero uniformly on $R^{n} \backslash B\left(R^{n}, 0, R\right)$ for each $m, j=0,1,2, \cdots$ and each $0<R<+\infty$, where $B(Z, z, R):=\{y \in Z: \rho(y, z) \leq R\}$ denotes a ball with center at $Z$ of radius $R$ in a metric space $Z$ with a metric $\rho$. The family of all $R$-linear and $A_{r}$-additive functionals on $B$ is denoted by $B^{\prime}$.

In particular we can take $X=A_{r}^{\alpha}, Y=A_{r}^{\beta}$ with $1 \leq \alpha, \beta \in Z$. Analogously spaces $D(U, Y)$,

$[D(U, Y)]^{\prime}, B(U, Y)$ and $[B(U, Y)]^{\prime}$ are defined for domains $U$ in $R^{n}$, for example, $U=U_{v}$ (see also $\S$ $1)$.

A generalized function $f \in B^{\prime}$ we call a generalized original, if there exist real numbers $a_{1}<a_{-1}$ such that for each $a_{1}<w_{-1}, w_{1}, \cdots, w_{-n}, w_{n}<a_{-1}$ the generalized function
4) $f(t) \exp \left(-\left(q_{v}, t\right)\right) \chi_{U_{v}}$ is in $\left[B\left(U_{v}, Y\right)\right]$ for all $v=\left(v_{1}, \cdots, v_{n}\right), v_{j} \in\{-1,1\}$ for every $j=1, \cdots, n$ for each $t \in R^{n}$ with $t_{j} v_{j} \geq 0$ for each $j=1, \cdots, n$, where $q_{v}=\left(v_{1} w_{v_{1}}, \cdots, v_{n} w_{v_{n} n}\right)$. By an image of such original we call a function.

5) $F^{n}(f, u ; p ; \zeta):=[f, \exp (-u(p, t ; \zeta)))$ of the variable $p \in A_{r}$ with the parameter $\zeta \in A_{r}$, defined in the domain $W_{f}=\left\{p \in A_{r}: a_{1}<\operatorname{Re}(p)<a_{-1}\right\}$ by the following rule. For a given $p \in W_{f}$ choose

$a_{1}<w_{1}, \cdots, w_{n}<\operatorname{Re}(p)<w_{-1}, \cdots, w_{-n}<a_{-1}$, then

6) $[f, \exp (-u(p, t ; \zeta))):=$

$$
\sum_{v}\left[f \exp \left(-\left(q_{v}, t\right)\right), \exp \left\{-\left[u(p, t ; \zeta)-\left(q_{v}, t\right)\right]\right\} \chi_{U_{v}}\right)
$$

since $\exp \left\{-\left[u(p, t ; \zeta)-\left(q_{v}, t\right)\right]\right\} \in B\left(U_{v}, Y\right)$,

where in each term

$$
\left[f \exp \left(-\left(q_{v}, t\right)\right), \exp \left\{-\left[u(p, t ; \zeta)-\left(q_{v}, t\right)\right]\right\} \chi_{U_{v}}\right)
$$

the generalized function belongs to $\left[B\left(U_{v}, Y\right)\right]^{\prime}$ by Condition (4), while the sum in (6) is by all admissible vectors $v \in\{-1,1\}^{n}$.

\subsection{Note and Examples}

Evidently the transform $F^{n}(f, u ; p ; \zeta)$ does not depend on a choice of $\left\{w_{-1}, w_{1}, \cdots, w_{-n}, w_{n}\right\}$, since

$$
\begin{aligned}
& {\left[f \exp \left(-\left(q_{v}, t\right), \exp \left(-\left[u(p, t ; \zeta)-\left(q_{v}, t\right)\right]\right) \chi_{U_{v}}\right)\right.} \\
& =\left[f \exp \left(-\left(q_{v}, t\right)-\left(b_{v}, t\right)\right), \exp \left(-\left[u(p, t ; \zeta)-\left(q_{v}, t\right)-\left(b_{v}, t\right)\right]\right) \chi_{U_{v}}\right)
\end{aligned}
$$

for each $b \in R^{n}$ such that

$$
a_{1}<w_{j}+b_{j}<\operatorname{Re}(p)<w_{-j}+b_{-j}<a_{-1}
$$

for each $j=1, \cdots, n$, because $\exp \left(-\left(b_{v}, t\right)\right) \in R$. At the same time the real field $R$ is the center of the Cayley-
Dickson algebra $A_{r}$, where $2 \leq r \in N$.

Let $\delta$ be the Dirac delta function, defined by the equation

$(D F)[\delta(t), \phi(t)):=\phi(0)$ for each $\phi \in B$. Then

1) $F^{n}\left(\delta^{(j)}(t-\tau), u ; p ; \zeta\right)=\sum_{v \in\{-1,\}^{n}}\left[\delta^{(j)}(t-\tau) \exp \left(-\left(q_{v}, t\right)\right), \exp \left(-\left[u(p, t ; \zeta)-\left(q_{v}, t\right)\right]\right) \chi_{U_{v}}\right)$

$$
=\left.(-1)^{j} \partial_{t}^{j} \exp (-[u(p, t ; \zeta)])\right|_{t=\tau},
$$

since it is possible to take $-\infty<a_{1}<0<a_{-1}<\infty$ and $w_{k}=0$ for each $k \in\{-1,1,-2,2, \cdots,-n, n\}$, where $\tau \in R^{n}$ is the parameter, $\partial_{t}^{j}:=\partial^{|j|} / \partial t_{1}^{j_{1}} \cdots \partial t_{1}^{j_{1}}$. In particular, for $j=0$ we have

2) $F^{n}(\delta(t-\tau), u ; p ; \zeta)=\exp (-u(p, \tau ; \zeta))$.

In the general case:

3) $F^{n}\left(\partial^{|j|} \delta(t) / \partial s_{1}^{j_{1}} \cdots \partial s_{n}^{j_{n}}, u ; p ; \zeta\right)=\sum_{0 \leq k_{1} \leq j_{1}}\left(\begin{array}{l}j_{1} \\ k_{1}\end{array}\right) p_{0}^{j_{1}-k_{1}}\left(p_{1} S_{e_{1}}\right)^{k_{1}}\left(p_{2} S_{e_{2}}\right)^{j_{2}} \cdots\left(p_{n} S_{e_{n}}\right)^{j_{n}} \exp \left(-\zeta_{0}-M(p, 0 ; \zeta)\right)$

in the $A_{r}$ spherical coordinates, or 
3.1) $F^{n}\left(\partial^{|j|} \delta(t) / \partial t_{1}^{j_{1}} \cdots \partial t_{n}^{j_{n}}, u ; p ; \zeta\right)=\left(p_{0}+p_{1} S_{e_{1}}\right)^{j_{1}}\left(p_{0}+p_{2} S_{e_{2}}\right)^{j_{2}} \cdots\left(p_{0}+p_{n} S_{e_{n}}\right)^{j_{n}} \exp (-u(p, 0 ; \zeta))$

in the $A_{r}$ Cartesian coordinates, where

$j_{1}+\cdots+j_{n}=|j|, k_{1}, j_{1}, \cdots, j_{n}$ are nonnegative integers, $2^{r-1} \leq n \leq 2^{r}-1,\left(\begin{array}{l}l \\ m\end{array}\right):=1 ! /[m !(l-m) !]$ denotes the binomial coefficient, $0 !=1,1 !=1,2 !=2$; $l !=1 \cdot 2 \cdots \cdots l$ for each $l \geq 3, s_{j}=s_{j}(n ; t)$.

The transform $F^{n}(f)$ of any generalized function $f$ is the holomorphic function by $p \in W_{f}$ and by $\zeta \in A_{r}$, since the right side of Equation 19(5) is holomorphic by $p$ in $W_{f}$ and by $\zeta$ in view of Theorem 4. Equation 19(5) implies, that Theorems 11-13 are accomplished also for generalized functions.

For $a_{1}=a_{-1}$ the region of convergence reduces to the vertical hyperplane in $A_{r}$ over $R$. For $a_{-1}<a_{1}$ there is no any common domain of convergence and $f(t)$ can not be transformed.

\subsection{Theorem}

If $f(t)$ is an original function on $R^{n}, F^{n}(p ; \zeta)$ is its image, $\partial^{|j|} f(t) / \partial s_{1}^{j_{1}} \cdots \partial s_{n}^{j_{n}}$ or $\partial^{|j|} f(t) / \partial t_{1}^{j_{1}} \cdots \partial t_{n}^{j_{n}}$ is an original, $|j|=j_{1}+\cdots+j_{n}, \quad 0 \leq j_{1}, \cdots, j_{n} \in Z$, $2^{r-1} \leq n \leq 2^{r}-1 ;$ then

1) $F^{n}\left(\partial^{|j|} f(t) / \partial s_{1}^{j_{1}} \cdots \partial s_{n}^{j_{n}}, u ; p ; \zeta\right)=\sum_{0 \leq k_{1} \leq j_{1}}\left(\begin{array}{l}j_{1} \\ k_{1}\end{array}\right) p_{0}^{j_{1}-k_{1}}\left(p_{1} S_{e_{1}}\right)^{k_{1}}\left(p_{2} S_{e_{2}}\right)^{j_{2}} \cdots\left(p_{n} S_{e_{n}}\right)^{j_{n}} F^{n}(f(t), u ; p ; \zeta)$

for $u(p, t ; \zeta):=p_{0} s_{1}+M(p, t ; \zeta)+\zeta_{0}$ given by $2(1,2,2.1)$, or

1.1) $F^{n}\left(\partial^{|j|} f(t) / \partial t_{1}^{j_{1}} \cdots \partial t_{n}^{j_{n}}, u ; p ; \zeta\right)=\left(p_{0}+p_{1} S_{e_{1}}\right)^{j_{1}}\left(p_{0}+p_{2} S_{e_{2}}\right)^{j_{2}} \cdots\left(p_{0}+p_{n} S_{e_{n}}\right)^{j_{n}} F^{n}(f(t), u ; p ; \zeta)$

for $u(p, t ; \zeta)$ given by $1(8,8.1)$ over the Cayley- Dickson algebra $A_{r}$ with $2 \leq r<\infty$. Domains, where Formulas $(1,1.1)$ are true may be different from a domain of the multiparameter noncommutative transform for $f$, but they are satisfied in the domain $a_{1}<\operatorname{Re}(p)<a_{-1}$, where

$$
\begin{gathered}
a_{-1}=\min \left(a_{-1}(f), a_{-1}\left(\partial^{|m|} f(t) / \partial \phi_{1}^{m_{1}} \cdots \partial \phi_{n}^{m_{n}}\right):|m| \leq|j|, 0 \leq m_{l} \leq j_{l} \forall l\right) \\
a_{1}=\max \left(a_{1}(f), a_{1}\left(\partial^{|m|} f(t) / \partial \phi_{1}^{m_{1}} \cdots \partial \phi_{n}^{m_{n}}\right):|m| \leq|k|, 0 \leq m_{l} \leq j_{l} \forall l\right),
\end{gathered}
$$

if $a_{1}<a_{-1}$, where $\phi_{j}=s_{j}$ or $\phi_{j}=t_{j}$ for each $j$ correspondingly.

metrically corresponds. The number of different vectors

Proof. To each domain $U_{v}$ the domain $U_{-v}$ sym$v \in\{-1,1\}^{n}$ is even $2^{n}$. Therefore, for $u=p_{0} t+\zeta_{0}+M(p, t ; \zeta)$ due to Theorem 12 the equality

2) $\int_{R^{n}}\left(\partial f(t) / \partial s_{j}\right) e^{-u(p, t ; \zeta)} \mathrm{d} s=\int_{R^{n}}\left(\partial f(t) / \partial s_{j}\right) e^{-u(p, t ; \zeta)} \mathrm{d} t$

$$
=\int_{R^{n-1}}\left(\mathrm{~d} t^{j}\right)\left[f(t) e^{-u(p, t ; \zeta)}\right]_{-\infty}^{\infty}-\int_{R^{n-1}}\left(\mathrm{~d} t^{j}\right)\left(\int_{-\infty}^{\infty} f(t)\left[\partial e^{-u(p, t ; \zeta)} / \partial s_{j}\right] \mathrm{d} s_{j}\right)
$$

is satisfied in the $A_{r}$ spherical coordinates, since the absolute value of the Jacobian $\partial t / \partial\left(t^{j}, s_{j}\right)$ is unit. Since for $a_{1}<\operatorname{Re}(p)<a_{-1}$ the first additive is zero, while the second integral converts with the help of Formulas 12(2,2.1), Formula (1) follows for $k=1$ :
3) $F^{n}\left(\partial f(t) / \partial s_{j}, u ; p ; \zeta\right)=$

$$
p_{0} \delta_{1, j} F^{n}(f(t), u ; p ; \zeta)+p_{j} S_{e_{j}} F^{n}(f(t), u ; p ; \zeta)
$$

To accomplish the derivation we use Theorem 14 so that

$$
\begin{aligned}
& \lim _{\tau \rightarrow 0}\left[F^{n}(f(t), u ; p ; \zeta)-F^{n}\left(f\left(t-\tau e_{j}\right), u ; p ; \zeta\right)\right] / \tau \\
& =\lim _{\tau \rightarrow 0}\left[F^{n}(f(t), u ; p ; \zeta)-F^{n}\left(f(t), u ; p ; \zeta+\tau\left(p_{0}+p_{1} i_{1}+\cdots+p_{j} i_{j}\right)\right)\right] / \tau \\
& =\lim _{\tau \rightarrow 0} \int_{R^{n}} f(t)\left[e^{-u(p, t ; \zeta)}-e^{-u\left(p, t ; \zeta+\tau\left(p_{0}+p_{1} i_{1}+\cdots+p_{j} i_{j}\right)\right)}\right] \tau^{-1} \mathrm{~d} t,
\end{aligned}
$$


where $e_{j}=(0, \cdots, 0,1,0, \cdots, 0) \in R^{n}$ with 1 on the $j$-th place. If the original $\partial^{|j|} f(t) / \partial s_{1}^{j_{1}} \cdots \partial s_{n}^{j_{n}}$ exists, then $\partial^{|m|} f(t) / \partial s_{1}^{m_{1}} \cdots \partial s_{n}^{m_{n}}$ is continuous for $0 \leq|m| \leq|j|-1$ with $0 \leq m_{l} \leq j_{l}$ for each $l=1, \cdots, n$, where $f^{0}:=f$. The interchanging of $\lim _{\tau \rightarrow 0}$ and $\int_{R^{n}}$ may change a domain of convergence, but in the indicated in the theorem domain $a_{1}<\operatorname{Re}(p)<a_{-1}$, when it is non void, Formula (3) is valid. Applying Formula (3) in the $A_{r}$ spherical coordinates by induction to

$$
\left(\partial^{|m|} f(t) / \partial s_{1}^{m_{1}} \cdots \partial s_{n}^{m_{n}}:|m| \leq|j|, 0 \leq m_{l} \leq j_{l} \forall l\right)
$$

with the corresponding order subordinated to $\partial^{|j|} f(t) / \partial s_{1}^{j_{1}} \cdots \partial s_{n}^{j_{n}}$, or in the $A_{r}$ Cartesian coordinates using Formula 12(1.1) for the partial derivatives

$$
\left.\left(\partial^{|m|} f(t) / \partial s_{1}^{m_{1}} \cdots \partial s_{n}^{m_{n}}\right):|m| \leq|j|, 0 \leq m_{l} \leq j_{l} \forall l\right)
$$

with the corresponding order subordinated to $\partial^{|j|} f(t) / \partial t_{1}^{j_{1}} \cdots \partial t_{n}^{j_{n}} \quad$ we deduce Expressions (1) and (1.1) with the help of Statement 6 from $\S$ XVII.2.3 [19] about the differentiation of an improper integral by a parameter and $\S 2$.

\subsection{Remarks}

For the entire Euclidean space $R^{n}$ Theorem 21 for $\partial f(t) / \partial s_{j}$ gives only one or two additives on the right side of 21(1) in accordance with 21(3).

Evidently Theorems 4, 11 and Proposition 10 are accomplished for $F^{k ; t_{j(1)}, \cdots, t_{j(k)}}(f, u ; p ; \zeta)$ also.

Theorem 12 is satisfied for $F^{k ; t_{j(1)}, \cdots, t_{j(k)}}$ and any $j \in\{j(1), \cdots, j(k)\}$, so that

$s_{l}=s_{l}(k ; t)=t_{j(l)}+\cdots+t_{j(k)} \quad$ for e a c h $1 \leq l \leq k$, $p_{m}=0$ and $\zeta_{m}=0$ for each $1 \leq m \notin\{j(1), \cdots, j(k)\}$ (the same convention is in $13,14,17,21$, see also below). For $F^{k ; t_{j(1)}, \cdots, t_{j(k)}}$ in Theorem 13 in Formula 13(1) it is natural to put $t_{m}=0$ and $h_{m}=0$ for each $1 \leq m \notin\{j(1), \cdots, j(k)\}$, so that only $(k+1)$ additives with $h_{0}, h_{j(1)}, \cdots, h_{j(k)}$ on the right side generally may remain. Theorems 14 and 17 and 21 modify for $F^{k ; t_{j(1)}, \cdots, t_{j(k)}}$ putting in 14(1) and 17(1,2) and 21(1)

$t_{j}=0$ and $\tau_{j}=0$ respectively for each $j \notin\{j(1), \cdots, j(k)\}$.

To take into account boundary conditions for domains different from $U_{v}$, for example, for bounded domains $V$ in $R^{n}$ we consider a bounded noncommutative multiparameter transform

1) $F^{n}\left(f(t) \chi_{V}, u ; p ; \zeta\right)=: F_{V}^{n}(f(t), u ; p ; \zeta)$.

For it evidently Theorems 4, 6-8, 11, 13, 14, 16, 17, Proposition 10 and Corollary 4.1 are satisfied as well taking specific originals $f$ with supports in $V$.

At first take domains $W$ which are quadrants, that is canonical closed subsets affine diffeomorphic with $Q^{n}=\prod_{j=1}^{n}\left[a_{j}, b_{j}\right]$, where $-\infty \leq a_{j}<b_{j} \leq \infty$, $\left[a_{j}, b_{j}\right]:=\left\{x \in R: a_{j} \leq x \leq b_{j}\right\}$ denotes the segment in $R$. This means that there exists a vector $w \in R^{n}$ and a linear invertible mapping $C$ on $R^{n}$ so that $C(W)-w=Q$. We put $t^{j, 1}:=\left(t_{1}, \cdots, t_{j}, \cdots, t_{n}: t_{j}=a_{j}\right)$, $t^{j, 2}:=\left(t_{1}, \cdots, t_{j}, \cdots, t_{n}: t_{j}=b_{j}\right)$. Consider $t=\left(t_{1}, \cdots, t_{n}\right) \in Q^{n}$.

\subsection{Theorem}

Let $f(t)$ be a function-original with a support by $t$ variables in $Q^{n}$ and zero outside $Q^{n}$ such that $\partial f(t) / \partial t_{j}$ also satisfies Conditions 1(1-4). Suppose that $u(p, t ; \zeta)$ is given by $2(1,2,2.1)$ or $1(8,8.1)$ over $A_{r}$ with $2 \leq r<\infty, 2^{r-1} \leq n \leq 2^{r}-1$. Then

$$
\text { 1) } \begin{aligned}
F^{n}\left(\left(\partial f(t) / \partial t_{j}\right) \chi_{Q^{n}}(t), u ; p ; \zeta\right)= & F^{n-1 ; t, j, 2}\left(f\left(t^{j, 2}\right) \chi_{Q^{n}}\left(t^{j, 2}\right), u ; p ; \zeta\right)-F^{n-1 ; t^{j, 1}}\left(f\left(t^{j, 1}\right) \chi_{Q^{n}}\left(t^{j, 1}\right), u ; p ; \zeta\right) \\
& +\left[p_{0}+\sum_{k=1}^{j} p_{k} S_{e_{k}}\right] F^{n}\left(f(t) \chi_{Q^{n}}(t), u ; p ; \zeta\right)
\end{aligned}
$$

in the $A_{r}$ spherical coordinates, or

$$
F^{n-1 ; t^{j, 2}}\left(f\left(t^{j, 2}\right) \chi_{Q^{n}}\left(t^{j, 2}\right), u ; p ; \zeta\right)-F^{n-1 ; t^{j, 1}}\left(f\left(t^{j, 1}\right) \chi_{Q^{n}}\left(t^{j, 1}\right), u ; p ; \zeta\right)+\left[p_{0}+p_{j} S_{e_{j}}\right] F^{n}\left(f(t) \chi_{Q^{n}}(t), u ; p ; \zeta\right)
$$

in the $A_{r}$ Cartesian coordinates in a domain $W \subset A_{r}$; if $a_{j}=-\infty$ or $b_{j}=+\infty$, then the addendum with $t^{j, 1}$ or $t^{j, 2}$ correspondingly is zero.

Proof. Here the domain $Q^{n}$ is bounded and $f$ is almost everywhere continuous and satisfies Conditions
1(1-4), hence

$$
f(t) \exp (-u(p, t ; \zeta)) \in L^{1}\left(R^{n}, \lambda_{n}, A_{r}\right)
$$

for each $p \in A_{r}$, since $\exp (-u(p, t ; \zeta))$ is continuous and $\operatorname{supp}(f(t)) \subset Q^{n}$.

Analogously to $\S 12$ the integration by parts gives

2) $\int_{a_{j}}^{b_{j}}\left(\partial f(t) / \partial t_{j}\right) \exp (-u(p, t ; \zeta)) \mathrm{d} t_{j}=\left.f(t) \exp (-u(p, t ; \zeta))\right|_{t_{j}=a_{j}} ^{t_{j}=b_{j}}-\int_{a_{j}}^{b_{j}}\left[f(t)\left(\partial \exp (-u(p, t ; \zeta)) / \partial t_{j}\right)\right] \mathrm{d} t_{j}$,

where $t=\left(t_{1}, \cdots, t_{n}\right)$. Then the Fubini's theorem implies: 
3) $\int_{Q^{n}}\left(\partial f(t) / \partial t_{j}\right) \exp (-u(p, t ; \zeta)) \mathrm{d} t=\int_{a_{1}}^{b_{1}} \cdots \int_{a_{j-1}}^{b_{j-1}} \int_{a_{j+1}}^{b_{j+1}} \int_{a_{n}}^{b_{n}}\left[\int_{a_{j}}^{b_{j}}\left(\partial f(t) / \partial t_{j}\right) \exp (-u(p, t ; \zeta)) \mathrm{d} t_{j}\right] \mathrm{d} t^{j}$

$$
\begin{aligned}
= & {\left[\int_{t \in Q^{n}, t_{j}=b_{j}} f\left(t^{j, 2}\right) \exp \left(-u\left(p, t^{j, 2} ; \zeta\right)\right) \mathrm{d} t^{j}\right]-\left[\int_{t \in Q^{n}, t_{j}=a_{j}} f\left(t^{j, 1}\right) \exp \left(-u\left(p, t^{j, 1} ; \zeta\right)\right) \mathrm{d} t^{j}\right] } \\
& +\left[p_{0}+\sum_{k=1}^{j} p_{k} S_{e_{k}}\right] \int_{a_{1}}^{b_{1}} \cdots \int_{a_{n}}^{b_{n}} f(t) \exp (-u(p, t ; \zeta)) \mathrm{d} t
\end{aligned}
$$

in the $A_{r}$ spherical coordinates or

3.1)

$$
\begin{aligned}
& \int_{Q^{n}}\left(\partial f(t) / \partial t_{j}\right) \exp (-u(p, t ; \zeta)) \mathrm{d} t \\
& =\left[\int_{t \in Q^{n}, t_{j}=b_{j}} f\left(t^{j, 2}\right) \exp \left(-u\left(p, t^{j, 2} ; \zeta\right)\right) \mathrm{d} t^{j}\right]-\left[\int_{t \in Q^{n}, t_{j}=a_{j}} f\left(t^{j, 1}\right) \exp \left(-u\left(p, t^{j, 1} ; \zeta\right)\right) \mathrm{d} t^{j}\right] \\
& \quad+\left[p_{0}+p_{j} S_{e_{j}}\right] \int_{a_{1}}^{b_{1}} \cdots \int_{a_{n}}^{b_{n}} f(t) \exp (-u(p, t ; \zeta)) \mathrm{d} t
\end{aligned}
$$

in the $A_{r}$ Cartesian coordinates, where as usually $t^{j}=\left(t_{1}, \cdots, t_{j-1}, 0, t_{j+1}, \cdots, t_{n}\right), \mathrm{d} t^{j}=\mathrm{d} t_{1} \cdots \mathrm{d} t_{j-1} \mathrm{~d} t_{j+1} \cdots \mathrm{d} t$. This gives Formulas $(1,1.1)$, where

4) $F^{n-1 ; t^{j, k}}\left(f\left(t^{j, k}\right) \chi_{Q^{n}}\left(t^{j, k}\right), u\left(p, t^{j, k} ; \zeta\right) ; p ; \zeta\right)$

$$
=\int_{a_{1}}^{b_{1}} \cdots \int_{a_{j-1}}^{b_{j-1}} \int_{a_{j+1}}^{b_{j+1}} \int_{a_{n}}^{b_{n}} f\left(t^{j, k}\right) \exp \left(-u\left(p, t^{j, k} ; \zeta\right)\right) \mathrm{d} t^{j, k}
$$

is the non-commutative transform by $t^{j, k}$, $2^{r-1} \leq n \leq 2^{r}-1, \mathrm{~d} t^{j, k}$ is the Lebesgue volume element on $R^{n-1}$.

\subsection{Theorem}

If a function $f(t) \chi_{Q^{n}}(t)$ is original together with its derivative $\partial^{n} f(t) \chi_{Q^{n}}^{Q^{n}}(t) / \partial s_{1} \cdots \partial s_{n}$ or $\partial^{n} f(t) \chi_{Q^{n}}(t) / \partial t_{1} \cdots \partial t_{n}$, where $F_{u}^{n}(p ; \zeta)$ is an image function of $f(t) \chi_{Q^{n}}(t)$ over the Cayley-Dickson algebra $A_{r}$ with $2 \leq r \in N, 2^{r-1} \leq n \leq 2^{r}-1$, for the function $u(p, t ; \zeta)$ given by $2(1,2,2.1)$ or $1(8,8.1)$, $Q^{n}=\prod_{j=1}^{n}\left[0, b_{j}\right], b_{j}>0$ for each $j$, then

$$
\text { 1) } \begin{aligned}
\lim _{p \rightarrow \infty}\left\{\left[p_{0}+p_{1} S_{e_{1}}\right] p_{2} S_{e_{2}} \cdots p_{n} S_{e_{n}} F_{u}^{n}(p ; \zeta)+\sum_{m=0}^{n-1}(-1)^{m}\right. \\
\left.\sum_{1 \leq j_{1}<\cdots<j_{n-m} \leq n ; 1 \leq \leq 1<\cdots<l_{m} \leq n ; l_{\alpha} \neq j_{\beta} \forall \alpha, \beta}\left[p_{0} \delta_{1, j_{1}}+p_{j_{1}} S_{e_{j_{1}}}\right] p_{j_{2}} S_{e_{j_{2}}} \cdots p_{j_{n-m}} S_{e_{j_{n-m}}} F_{u}^{n-m}\left(p^{(l)} ; \zeta\right)\right\}=(-1)^{n+1} f(0) e^{-u(0,0 ; \zeta)}
\end{aligned}
$$

in the $A_{r}$ spherical coordinates, or

$$
\begin{aligned}
\text { 1.1) } \lim _{p \rightarrow \infty}\left\{\left[p_{0}+p_{1} S_{e_{1}}\right]\left[p_{0}+p_{2} S_{e_{2}}\right] \cdots\left[p_{0}+p_{n} S_{e_{n}}\right] F_{u}^{n}(p ; \zeta)+\sum_{m=0}^{n-1}(-1)^{m}\right. \\
\left.\sum_{1 \leq j_{1}<\cdots<j_{n-m} \leq n ; 1 \leq l_{1}<\cdots<l_{m} \leq n ; l_{\alpha} \neq j_{\beta} \forall \alpha, \beta}\left[p_{0}+p_{j_{1}} S_{e_{j_{1}}}\right]\left[p_{0}+p_{j_{2}} S_{e_{j_{2}}}\right] \cdots\left[p_{0}+p_{j_{n-m}} S_{e_{j_{n-m}}}\right] F_{u}^{n-m}\left(p^{(l)} ; \zeta\right)\right\}=(-1)^{n+1} f(0) e^{-u(0,0 ; \zeta)}
\end{aligned}
$$

in the $A_{r}$ Cartesian coordinates, where

$$
f(0)=\lim _{t \in Q^{n}, t \rightarrow 0} f(t),
$$

$p$ tends to the infinity inside the angle

2) $F^{n-1 ; t^{j, k}}\left(f\left(t^{j, k}\right) \chi_{Q^{n}}\left(t^{j, k}\right), u\left(p, t^{j, k} ; \zeta\right) ; p ; \zeta\right)$

$$
=\lim _{t_{j} \rightarrow \beta_{j, k}+0} \int_{a_{1}}^{b_{1}} \mathrm{~d} t_{1} \cdots \int_{a_{j-1}}^{b_{j-1}} \mathrm{~d} t_{j-1} \int_{a_{j+1}}^{b_{j+1}} \mathrm{~d} t_{j+1} \cdots \int_{a_{n}}^{b_{n}}\left(\mathrm{~d} t_{n}\right) f(t) \exp (-u(p, t ; \zeta)),
$$

where $\beta_{j, 1}=a_{j}=0, \beta_{j, 2}=b_{j}>0, k=1,2$. Mention, that

$$
\left(\cdots\left(\left(t^{1, l_{1}}\right)^{2, l_{2}}\right) \cdots\right)^{j, l_{j}}=\left(t: t_{1}=\beta_{1, l_{1}}, \cdots, t_{j}=\beta_{j, l_{j}}\right)
$$

for every $1 \leq j \leq n$. Analogously to $\S 12$ we apply
Formula (2) by induction $j=1, \cdots, n, 2^{r-1} \leq n \leq 2^{r}-1$, to

$$
\begin{aligned}
& \partial^{n} f(t(s)) / \partial s_{1} \cdots \partial s_{n}, \cdots, \partial^{n-j+1} f(t(s)) / \partial s_{j} \cdots \partial s_{n} \\
& , \cdots, \partial f(t(s)) / \partial s_{n}
\end{aligned}
$$


instead of $\partial f(t(s)) / \partial s_{j}, s_{j}=s_{j}(n ; t)$ as in $\S 2$, or applying to the partial derivatives

$$
\partial^{n} f(t) / \partial t_{1} \cdots \partial t_{n}, \cdots, \partial^{n-j+1} f(t) / \partial t_{j} \cdots \partial t_{n}, \cdots, \partial f(t) / \partial t_{n}
$$

instead of $\partial f(t) / \partial t_{j}$ correspondingly. If $s_{j}>0$ for some $j \geq 1$, then $s_{1}>0$ for $Q^{n}$ and

$\lim _{p \rightarrow \infty} e^{-u\left(p, t^{(l)} ; \zeta\right)}=0$ for such $t^{(l)}$, where

$t=\left(t_{1}, \cdots, t_{n}\right),(l)=\left(l_{1}, \cdots, l_{n}\right),|l|=l_{1}+\cdots+l_{n}$,

$t^{(l)}=\left(t_{1}^{(l)}, \cdots, t_{n}^{(l)}\right), \quad t_{j}^{(l)}=a_{j}$ for $l_{j}=1$ and $t_{j}^{(l)}=b_{j}$ for $l_{j}=2,1 \leq j \leq 2^{r}-1$. Therefore,

$$
\begin{aligned}
& \lim _{p \rightarrow \infty} \sum_{l_{j} \in\{1,2\} ; j=1, \cdots, n}(-1)^{|l|} f\left(t^{(l)}\right) e^{-u\left(p, t^{(l)} ; \zeta\right)} \\
& =(-1)^{n} f(0) e^{-u(0,0 ; \zeta)},
\end{aligned}
$$

since $u(p, 0 ; \zeta)=u(0,0 ; \zeta)$, where

$$
f\left({ }^{(l)}\right)=\lim _{t \in Q^{n} ; t \rightarrow t^{(l)}} f(t) .
$$

In accordance with Note 8 [4]

$$
\lim _{p \rightarrow \infty,|\operatorname{Arg}(p)|<\pi / 2-\delta} F^{n}\left(\left(\partial^{n} f(t) / \partial s_{1} \cdots \partial s_{n}\right) \chi_{Q^{n}}(t), u(p, t ; \zeta) ; p ; \zeta\right)=0
$$

in the $A_{r}$ spherical coordinates and

$$
\lim _{p \rightarrow \infty,|A r g(p)|<\pi / 2-\delta} F^{n}\left(\left(\partial^{n} f(t) / \partial t_{1} \cdots \partial t_{n}\right) \chi_{Q^{n}}(t), u(p, t ; \zeta) ; p ; \zeta\right)=0
$$

in the $A_{r}$ Cartesian coordinates, which gives the statement of this theorem.

\subsection{Theorem}

Suppose that $f(t) \chi_{Q^{n}}(t)$ is an original function, $F^{n}(p ; \zeta)$ is its image, $\partial^{|j|} f(t) \chi_{Q^{n}}(t) / \partial t_{1}^{j_{1}} \cdots \partial t_{n}^{j_{n}}$ is an original, $|j|=j_{1}+\cdots+j_{n}, \quad 0 \leq j_{1}, \cdots, j_{n} \in Z$, $2^{r-1} \leq n \leq 2^{r}-1,-\infty \leq a_{k}<b_{k} \leq \infty$ for each $k=1, \cdots, n, \quad(l)=\left(l_{1}, \cdots, l_{n}\right), \quad l_{k} \in\{0,1,2\}, W=A_{r}$ for bounded $Q^{n}$. Let $W=\left\{p \in A_{r}: a_{1}<\operatorname{Re}(p)\right\}$ for $b_{k}=\infty$ for some $k$ and finite $a_{k}$ for each $k$; $W=\left\{p \in A_{r}: \operatorname{Re}(p)<a_{-1}\right\}$ for $a_{k}=-\infty$ for some $k$ and finite $b_{k}$ for each $k$;

$W=\left\{p \in A_{r}: a_{1}<\operatorname{Re}(p)<a_{-1}\right\} \quad$ when $a_{k}=-\infty \quad$ and $b_{l}=+\infty$ for some $k$ and $l ; t^{(l)}=\left(t_{1}^{(l)}, \cdots, t_{n}^{(l)}\right)$.

We put $t_{k}^{(l)}=t_{k}$ and $q_{k}=0$ for $l_{k}=0, t_{k}^{(l)}=a_{k}$ for $l_{k}=1, \quad t_{k}^{(l)}=b_{k}$ for $l_{k}=2, \quad(q)=\left(q_{1}, \cdots, q_{n}\right)$, $|q|=q_{1}+\cdots+q_{n}$,

$$
\begin{gathered}
a_{1}=\max \left(a_{1}(f), a_{1}\left(\partial^{|m|} f(t) / \partial t_{1}^{m_{1}} \cdots \partial t_{n}^{m_{n}}\right):|m| \leq|j|, 0 \leq m_{k} \leq j_{k} \forall k\right), \\
a_{-1}=\min \left(a_{-1}(f), a_{-1}\left(\partial^{|m|} f(t) / \partial t_{1}^{m_{1}} \cdots \partial t_{n}^{m_{n}}\right):|m| \leq|j|, 0 \leq m_{k} \leq j_{k} \forall k\right) \text { if } a_{1}<a_{-1} .
\end{gathered}
$$

If $a_{k}=-\infty$ and $b_{k}=+\infty$ for $Q^{n}$ with a given $k$, then $l_{k}=0$. If either $a_{k}>-\infty$ or $b_{k}<+\infty$ for a marked $k$, then $l_{k} \in\{0,1,2\}$. We also put $h_{k}=h_{k}(l)=\operatorname{sign}\left(l_{k}\right)$ for each $k$, where $\operatorname{sign}(x)=-1$ for $x<0, \operatorname{sign}(0)=0, \operatorname{sign}(x)=1$ for $x>0$, $h=h(l),|h|=\left|h_{1}\right|+\cdots+\left|h_{n}\right|$,

$$
(l j):=\left(l_{1} \operatorname{sign}\left(j_{1}\right), \cdots, l_{n} \operatorname{sign}\left(j_{n}\right)\right) .
$$

Let the vector $(l)$ enumerate faces $\partial Q_{(l)}^{n}$ in $\partial Q_{k-1}^{n}$ for $|h(l)|=k \geq 1$, so that $\partial Q_{k-1}^{n}=\bigcup_{\mid h(l)=k} Q_{(l)}^{n}$, $\partial Q_{(l)}^{n} \cap \partial Q_{(m)}^{n}=\varnothing$ for each $(l) \neq(m)$ (see also more detailed notations in $\S 28$ ).

Let the shift operator be defined:

$$
T_{(m)} F(p ; \zeta):=F\left(p ; \zeta-\left(i_{1} m_{1}+\cdots+i_{n} m_{n}\right) \pi / 2\right),
$$

also the operator $a_{k}>-\infty$

$$
\text { (SO) } S_{(m)} F(p ; \zeta):=S_{e_{1}}^{m_{1}} \cdots S_{e_{n}}^{m_{n}} F(p ; \zeta),
$$

where $(m)=\left(m_{1}, \cdots, m_{n}\right) \in[0, \infty)^{n} \subset R^{n}, \quad S_{(m)}^{k}=S_{k(m)}$ for each positive number $0<k \in R, S_{0}=I$ is the unit operator for $(m)=0$ (see also Formulas 12(3.1-3.7)). As usually let $e_{1}=(1,0, \cdots, 0), \cdots, e_{n}=(0, \cdots, 0,1)$ be the standard orthonormal basis in $R^{n}$ so that $(m)=m_{1} e_{1}+\cdots+m_{n} e_{n}$.

Theorem. Then

$$
\begin{aligned}
& \text { 1) } F^{n}\left(\partial^{|j|} f(t) \chi_{Q^{n}}(t) / \partial t_{1}^{j_{1}} \cdots \partial t_{n}^{j_{n}}, u(p, t ; \zeta) ; p ; \zeta\right)=R_{e_{1}}^{j_{1}} R_{e_{2}}^{j_{2}} \cdots R_{e_{n}}^{j_{n}} F^{n}\left(f(t) \chi_{Q^{n}}(t), u ; p ; \zeta\right) \\
&+\sum_{1 \leq(l j) ; m_{k}+q_{k}+h_{k}=j_{k} ; 0 \leq m_{k} ; 0 \leq q_{k} ; \eta_{k}=\operatorname{sign}\left(l_{k} j_{k}\right) ; q_{k}=0 \text { for } l_{k} j_{k}=0, \text { for each } k=1, \cdots, n ;(l) \in\{0,1,2\}^{n}} \\
&(-1)^{(l j) \mid} R_{e_{1}}^{m_{1}} R_{e_{2}}^{m_{2}} \cdots R_{e_{n}}^{m_{n}} F^{n-|h(j)|}\left(\partial^{|q|} f\left(t^{(l j)}\right) \chi_{\partial Q_{(j j)}^{n}}\left(t^{(l j)}\right) / \partial t_{1}^{q_{1}} \cdots \partial t_{n}^{q_{n}}, u ; p ; \zeta\right)
\end{aligned}
$$


for $u(p, t ; \zeta)$ in the $A_{r}$ spherical coordinates or the $A_{r}$ Cartesian coordinates over the Cayley-Dickson algebra $A_{r}$ with $2 \leq r<\infty$, where

$$
\text { 1.1) } \begin{aligned}
& R_{e_{2}}:=p_{0}+p_{1} S_{e_{1}}+p_{2} S_{e_{2}}, \cdots, \\
& R_{e_{n}}:=p_{0}+p_{1} S_{e_{1}}+p_{2} S_{e_{2}}+\cdots+p_{n} S_{e_{n}}
\end{aligned}
$$

in the $A_{r}$ spherical coordinates, while
1.2), $R_{e_{2}}:=p_{0}+p_{2} S_{e_{2}}, \cdots, R_{e_{n}}:=p_{0}+p_{n} S_{e_{n}}$ in the $A_{r}$ Cartesian coordinates, i.e. $R_{e_{j}}=R_{e_{j}}(p)$ are operators depending on the parameter $p$. If $t_{j}^{(l)}=\infty$ for some $1 \leq j \leq n$, then the corresponding addendum on the right of (1) is zero.

Proof. In view of Theorem 23 we get the equality

2) $\int_{Q^{n}}\left[\left(\partial^{|m|+1} f(t) / \partial t_{1}^{m_{1}} \cdots \partial t_{k-1}^{m_{k-1}} \partial t_{k}^{m_{k}+1} \partial t_{k+1}^{m_{k+1}} \cdots \partial t_{n}^{m_{n}}\right) e^{-u(p, t ; \zeta)}\right] \mathrm{d} t$

$$
=\left.\int_{R^{n-1} \cap Q^{n}}\left(\mathrm{~d} t^{k}\right)\left[\left(\partial^{|m|} f(t) / \partial t_{1}^{m_{1}} \cdots \partial t_{n}^{m_{n}}\right) e^{-u(p, t ; \zeta)}\right]\right|_{a_{k}} ^{b_{k}}-\int_{R^{n-1} \cap Q^{n}}\left(\mathrm{~d} t^{k}\right)\left(\int_{a_{k}}^{b_{k}}\left(\partial^{|m|} f(t) / \partial t_{1}^{m_{1}} \cdots \partial t_{n}^{m_{n}}\right)\left[\partial e^{-u(p, t ; \zeta)} / \partial t_{k}\right] \mathrm{d} t_{k}\right)
$$

is satisfied for $0 \leq m_{k} \leq j_{k}$ for each $k=1, \cdots, n$ with $|m|<|j|$. On the other hand, for $p \in W$ additives on the right of (2) convert with the help of Formula 23(1). Each term of the form

$$
\begin{aligned}
& \int_{R^{n-|h(l)|} \cap Q^{n}}\left(\mathrm{~d} t^{(l)}\right) \\
& {\left[\left(\partial^{|q|} f\left(t^{(l)}\right) \chi_{\partial Q_{(l)}^{n}}\left(t^{(l)}\right)\right) / \partial t_{1}^{q_{1}} \cdots \partial t_{n}^{q_{n}} e^{-u(p, t ; \zeta)}\right]}
\end{aligned}
$$

can be further transformed with the help of (2) by the considered variable $t_{k}$ only in the case $l_{k}=0$.

Applying Formula (2) by induction to partial derivatives $\partial^{|j|} f / \partial t_{1}^{j_{1}} \cdots \partial t_{n}^{j_{n}}, \partial^{|j|-j_{1}} f / \partial t_{2}^{j_{2}} \cdots \partial t_{n}^{j_{n}}, \cdots$, $\partial^{j_{n}} f / \partial t_{n}^{j_{n}}, \cdots, \partial f / \partial t_{n}$ as in $\S 21$ and using Theorem
14 and Remarks 22 we deduce (1).

\subsection{Theorem}

Let $f(t) \chi_{U_{1, \ldots 1}}(t)$ be a function-original with values in $A_{r}$ with $2 \leq r<\infty, \quad 2^{r-1} \leq n \leq 2^{r}-1, u$ is given by $2(1,2,2.1)$ or $1(8,8.1)$,

1) $g(t):=\int_{0}^{t_{1}} \cdots \int_{0}^{t_{n}} f(x) \mathrm{d} x$, then

2) $F^{n}\left(f \chi_{U_{1, \cdots, 1}}(t), u ; p ; \zeta\right)$

$$
=R_{e_{1}} R_{e_{2}} \cdots R_{e_{n}} F^{n}\left(g(t) \chi_{U_{1, \cdots, 1}}(t), u ; p ; \zeta\right)
$$

in the domain $\operatorname{Re}(p)>\max \left(a_{1}, 0\right)$, where the operators $R_{e_{1}}$ are given by Formulas $25(1,1,1.2)$.

Proof. In view of Theorem 25 the equation

3) $F^{n}\left(f \chi_{U_{1, \cdots, 1}}(t), u ; p ; \zeta\right)=R_{e_{1}} R_{e_{2}} \cdots R_{e_{n}} F^{n}(g(t), u ; p ; \zeta)$

$$
+\sum_{1 \leq l \mid ; 0 \leq m_{k} \leq 1 ; m_{k}+h_{k}=1 ; h_{k}=\operatorname{sign}\left(l_{k}\right) ; \text { for each } k=1, \cdots, n ; q_{1}=0, \cdots, q_{n}=0}(-1)^{|(l)|} R_{e_{1}}^{m_{1}} R_{e_{2}}^{m_{2}} \cdots R_{e_{n}}^{m_{n}} F^{n-|h(l)|}\left(g\left(t^{(l)}\right), u ; p ; \zeta\right),
$$

is satisfied, since $\partial^{n} g(t) / \partial t_{1} \cdots \partial t_{n}=\left(f \chi_{U_{1} \ldots, 1}\right)(t)$, where $j_{1}=1, \cdots, j_{n}=1, l_{j}=1$ for each $j=1, \cdots, n$. Equation (3) is accomplished in the same domain

$\operatorname{Re}(p)>\max \left(a_{1}, 0\right)$, since $g(0)=0$ and $g(t)$ also fulfills conditions of Definition 1 , while

$a_{1}(g)<\max \left(a_{1}(f), 0\right)+b$ for each $b>0$, where $a_{1} \in R$. On the other hand, $g(t)$ is equal to zero on $\partial U_{1, \ldots, 1}$ and outside $U_{1, \ldots, 1}$ in accordance with formula (1), hence all terms on the right side of Equation (3) with $|l|>0$ disappear and $\operatorname{supp}(g(t)) \subset U_{1, \cdots, 1}$. Thus we get Equation (2).

\subsection{Theorem}

Suppose that $F^{k}(p ; \zeta)$ is an image $F^{k ; t_{1}, \cdots, t_{k}}\left(f(t) \chi_{U_{1}, \ldots, 1}(t), u ; p ; \zeta\right)$ of an original function $f(t)$ for $u$ given by $2(1,2,2.1)$ in the half space

$W:=\left\{p \in A_{r}: \operatorname{Re}(p)>a_{1}\right\}$ with $2 \leq r<\infty$,

$p_{1}=0, \cdots, p_{j-1}=0 ; \zeta_{1}=\pi / 2, \cdots, \zeta_{j-1}=\pi / 2$ for each $j \geq 2$ in the $A_{r}$ spherical coordinates or $\zeta_{1}=0, \cdots, \zeta_{j-1}=0$ for each $j \geq 2$ in the $A_{r}$ Cartesian coordinates;

1) the integral $\int_{p_{i} i_{j}}^{\infty i_{j}} F^{k}\left(p_{0}+z ; \zeta\right) \mathrm{d} z$ converges, where $p=p_{0}+p_{1} i_{1} i_{i}+\cdots+p_{k} i_{k} \in A_{r}, \quad p_{j} \in R$ for each $j=0, \cdots, 2^{r}-1,2^{r-1} \leq k \leq 2^{r}-1$, $U_{1, \cdots, 1}:=\left\{\left(t_{1}, \cdots, t_{k}\right) \in R^{k}: t_{1} \geq 0, \cdots, t_{k} \geq 0\right\}$. Let also

2) the function $F^{k}(p ; \zeta)$ be continuous by the variable $p \in A_{r}$ on the open domain $W$, moreover, for each $w>a_{1}$ there exist constants $C_{w}^{\prime}>0$ and $\varepsilon_{w}>0$ such that

3) $\left|F^{k}(p ; \zeta)\right| \leq C_{w}{ }^{\prime} \exp \left(-\varepsilon_{w}|p|\right)$ for each $p \in S_{R(n)}$, $S_{R}:=\left\{z \in A_{r}: \operatorname{Re}(z) \geq w\right\}, 0<R(n)<R(n+1)$ for each $n \in N, \lim _{n \rightarrow \infty} R(n)=\infty$, where $a_{1}$ is fixed, $\zeta=\zeta_{0} i_{0}+\cdots+\zeta_{k} i_{k} \in A_{r}$ is marked, $\zeta_{j} \in R$ for each $j=0, \cdots, k$. Then

$$
\text { 4) } \begin{aligned}
& \int_{p_{j} i_{j}}^{\infty i_{j}} F^{k}\left(p_{0}+z ; \zeta\right) \mathrm{d} z= \\
& S_{-e_{j}} F^{k: t_{1}, \ldots, t_{k}}\left(f(t) \chi_{U_{1, \cdots, 1}}(t) / \xi_{j}, u ; p ; \zeta\right),
\end{aligned}
$$


where $p_{1}=0, \cdots, p_{j-1}=0$ for each $j \geq 2$;

$\zeta_{1}=\pi / 2, \cdots, \zeta_{j-1}=\pi / 2$ and $\xi_{j}=s_{j}(k ; t)$ in the $A_{r}$ spherical coordinates, while $\zeta_{1}=0, \cdots, \zeta_{j-1}=0$ and $\xi_{j}=t_{j}$ in the $A_{r}$ Cartesian coordinates correspondingly for each $j \geq 1$.

Proof. Take a path of an integration belonging to the half space $\operatorname{Re}(p) \geq w$ for some constant $w>a_{1}$. Then

$$
\begin{aligned}
& \left|\int_{U_{1}, \cdots, 1} f(t) \exp (-u(p, t ; \zeta)) \mathrm{d} t\right| \\
& \leq C \int_{U_{1, \cdots, 1}} \exp \left(-\left(p_{0}-a_{1}\right)\left(t_{1}+\cdots+t_{k}\right)\right) \mathrm{d} t<\infty
\end{aligned}
$$

converges, where $C=$ const $>0, p_{0} \geq w$. For $t_{j}>0$ for each $j=1, \cdots, k$ conditions of Lemma 2.23 [4] (that is of the noncommutative analog over $A_{r}$ of Jordan's lemma) are satisfied. If $t_{j} \rightarrow \infty$, then $s_{j} \rightarrow \infty$, since all $t_{1}, \cdots, t_{k}$ are non-negative. Up to a set $\partial U_{1, \cdots, 1}$ of $\lambda_{k}$ Lebesgue measure zero we can consider that

$t_{1}>0, \cdots, t_{k}>0$. If $s_{j} \rightarrow \infty$, then also $s_{1} \rightarrow \infty$. The converging integral can be written as the following limit:

$$
\text { 5) } \begin{aligned}
& \int_{p_{j} i_{j}}^{\infty i_{j}} F^{k}\left(p_{0}+z ; \zeta\right) \mathrm{d} z \\
= & \lim _{0<\kappa \rightarrow 0} \int_{p_{j} i_{j}}^{\infty i_{j}} F^{k}\left(p_{0}+z ; \zeta\right) \exp (-\kappa|z|) \mathrm{d} z
\end{aligned}
$$

for $1 \leq j \leq k$, since the integral $\int_{-S \infty}^{S \infty}\left[F^{k}(w+z ; \zeta)\right] \mathrm{d} z$ is absolutely converging and the limit

$\lim _{\kappa \rightarrow 0} \exp (-\kappa|z|)=1$ uniformly by $z$ on each compact subset in $A_{r}$, where $S$ is a purely imaginary marked Cayley-Dickson number with $|S|=1$. Therefore, in the integral

6) $\int_{p_{j} i_{j}}^{\infty i_{j}} F^{k}\left(p_{0}+z ; \zeta\right) \mathrm{d} z=$

$$
\int_{p_{j} i_{j}}^{\infty i_{j}}\left(\int_{U_{1, \cdots, 1}} f(t)\left[\exp \left(-u\left(p_{0}+z, t ; \zeta\right)\right)\right] \mathrm{d} t\right) \mathrm{d} z
$$

the order of the integration can be changed in accordance with the Fubini's theorem applied componentwise to an integrand $g=g_{0} i_{0}+\cdots+g_{n} i_{n}$ with $g_{l} \in R$ for each $l=0, \cdots, n$ :

$$
\text { 7) } \begin{aligned}
& \int_{p_{j} i_{j}}^{\infty i_{j}} F^{k}\left(p_{0}+z ; \zeta\right) \mathrm{d} z \\
= & \int_{U_{1, \cdots, 1}} \mathrm{~d} t\left(\int_{p_{j} i_{j}}^{\infty i_{j}} f(t) \exp \left(-u\left(p_{0}+z, t ; \zeta\right)\right) \mathrm{d} z\right) \\
= & \int_{U_{1, \cdots, 1}} f(t)\left\{\int_{p_{j} i_{j}}^{\infty i_{j}}\left[e^{-u\left(p_{0}+z, t ; \zeta\right)}\right] \mathrm{d} z\right\} \mathrm{d} t .
\end{aligned}
$$

Generally, the condition $p_{1}=0, \cdots, p_{j-1}=0$ and $\zeta_{1}=\pi / 2, \cdots, \zeta_{j-1}=\pi / 2$ in the $A_{r}$ spherical coordinates or $\zeta_{1}=0, \cdots, \zeta_{j-1}=0$ in the $A_{r}$ Cartesian coordinates for each $j \geq 2$ is essential for the convergence of such integral. We certainly have
8) $\int_{p_{j} i_{j}}^{b_{j} i_{j}} \cos \left(i_{j}^{*} z \xi_{j}+\zeta_{j}\right) \mathrm{d} z$

$$
\begin{aligned}
& =\left.\left[\sin \left(\theta_{j} \xi_{j}+\zeta_{j}\right) / \xi_{j}\right]\right|_{\theta_{j}=p_{j}} ^{\theta_{j}=b_{j}} \\
& =\left.\left[-\cos \left(\theta_{j} \xi_{j}+\zeta_{j}+\pi / 2\right) / \xi_{j}\right]\right|_{\theta_{j}=p_{j}} ^{\theta_{j}=b_{j}}
\end{aligned}
$$

and

9) $\int_{p_{j} i_{j}}^{b_{j} i_{j}} \sin \left(i_{j}^{*} z \xi_{j}+\zeta_{j}\right) \mathrm{d} z_{j}$

$$
\begin{aligned}
& =\left.\left[-\cos \left(\theta_{j} \xi_{j}+\zeta_{j}\right) / \xi_{j}\right]\right|_{\theta_{j}=p_{j}} ^{\theta_{j}=b_{j}} \\
& =\left.\left[-\sin \left(\theta_{j} \xi_{j}+\zeta_{j}+\pi / 2\right) / \xi_{j}\right]\right|_{\theta_{j}=p_{j}} ^{\theta_{j}=b_{j}}
\end{aligned}
$$

for each $\xi_{j}>0$ and $-\infty<p_{j}<b_{j}<\infty$ and $j=1, \cdots, k$. Applying Formulas (3-9) and 2(1,2,2.1) or $1(8,8.1)$ and $12(3.1-3.7)$ we deduce that:

$$
\begin{aligned}
& \int_{p_{j} i_{j}}^{\infty i_{j}}\left[F^{k}\left(p_{0}+z ; \zeta\right)\right] \mathrm{d} z \\
& =S_{-e_{j}} \int_{U_{1, \cdots, 1}}\left[f(t) / \xi_{j}\right] \exp \{-u(p, t ; \zeta)\} \mathrm{d} t \\
& =S_{-e_{j}} F^{k ; t_{1}, \cdots, t_{k}}\left(f(t) \chi_{U_{1, \cdots, 1}}(t) / \xi_{j}, u ; p ; \zeta\right),
\end{aligned}
$$

where $t=\left(t_{1}, \cdots, t_{k}\right), s_{j}=t_{j}+\cdots+t_{k}$ for each $1 \leq j<k, s_{k}=t_{k}, \quad \xi_{j}=s_{j}$ in the $A_{r}$ spherical coordinates or $\xi_{j}=t_{j}$ in the $A_{r}$ Cartesian coordinates.

\subsection{Application of the Noncommutative Multiparameter Transform to Partial Differential Equations}

Consider a partial differential equation of the form:

1) $A[f](t)=g(t)$, where

2) $A[f](t):=\sum_{|j| \leq \alpha} a_{j}(t)\left(\partial^{|j|} f(t) / \partial t_{1}^{j_{1}} \cdots \partial t_{n}^{j_{n}}\right)$,

$a_{j}(t) \in A_{\kappa}$ are continuous functions, where $0 \leq \kappa \in Z$, $j=\left(j_{1}, \cdots, j_{n}\right),|j|:=j_{1}+\cdots+j_{n}, \quad 0 \leq j_{k} \in Z, \alpha$ is a natural order of a differential operator $A, 2 \leq r$, $2^{r-1} \leq n \leq 2^{r}-1$. Since

$s_{k}=s_{k}(n ; t)=t_{k}+\cdots+t_{n}$ for each $k=1, \cdots, n$, the operator $A$ can be rewritten in $s$ coordinates as

2.1) $A[f](t(s))$

$$
:=\sum_{|j| \leq \alpha} b_{j}(t)\left(\partial^{|j|} f(t(s)) / \partial s_{1}^{j_{1}} \cdots \partial s_{n}^{j_{n}}\right) .
$$

That is, there exists $b_{j} \neq 0$ for some $j$ with $|j|=\alpha$ and $b_{j}=0$ for $|j|>\alpha$, while a function $\sum_{j, \mid j=\alpha} b_{j}(t(s)) s_{1}^{j_{1}} \cdots s_{n}^{j_{n}}$ is not zero identically on the corresponding domain $V$. We consider that

(D1) $U$ is a canonical closed subset in the Euclidean space $R^{n}$, that is $U=c l(\operatorname{Int}(U))$, where $\operatorname{Int}(U)$ denotes the interior of $U$ and $\operatorname{cl}(U)$ denotes the closure of $U$.

Particularly, the entire space $R^{n}$ may also be taken. 
Under the linear mapping $\left(t_{1}, \cdots, t_{n}\right) \mapsto\left(s_{1}, \cdots, s_{n}\right)$ the domain $U$ transforms onto $V$.

We consider a manifold $W$ satisfying the following conditions (i-v).

i). The manifold $W$ is continuous and piecewise $C^{\alpha}$, where $C^{l}$ denotes the family of $l$ times continuously differentiable functions. This means by the definition that $W$ as the manifold is of class $C^{0} \cap C_{l o c}^{\alpha}$. That is $W$ is of class $C^{\alpha}$ on open subsets $W_{0, j}$ in $W$ and $W \backslash\left(\bigcup_{j} W_{0, j}\right)$ has a codimension not less than one in $W$.

ii). $W=\bigcup_{j=0}^{m} W_{j}$, where $W_{0}=\bigcup_{k} W_{0, k}, W_{j} \cap W_{k}=\varnothing$ for each $k \neq j, \quad m=\operatorname{dim}_{R} W, \quad \operatorname{dim}_{R} W_{j}=m-j$, $W_{j+1} \subset \partial W_{j}$.

iii). Each $W_{i}$ with $j=0, \cdots, m-1$ is an oriented $C^{\alpha}$ manifold, $W_{j}$ is open in $\bigcup_{k=j}^{m} W_{k}$. An orientation of $W_{j+1}$ is consistent with that of $\partial W_{j}$ for each

$j=0,1, \cdots, m-2$. For $j>0$ the set $W_{j}$ is allowed to be void or non-void.

iv). A sequence $W^{k}$ of $C^{\alpha}$ orientable manifolds embedded into $R^{n}, \alpha \geq 1$, exists such that $W^{k}$ uniformly converges to $W$ on each compact subset in $R^{n}$ relative to the metric dist.

For two subsets $B$ and $E$ in a metric space $X$ with a metric $\rho$ we put

3) $\operatorname{dist}(B, E):=$

$$
\max \left\{\sup _{b \in B} \operatorname{dist}(\{b\}, E), \sup _{e \in E} \operatorname{dist}(B,\{e\})\right\},
$$

where $\operatorname{dist}(\{b\}, E):=\inf _{e \in E} \rho(b, e)$, $\operatorname{dist}(B,\{e\}):=\inf _{b \in B} \rho(b, e), b \in B, e \in E$.

Generally, $\operatorname{dim}_{R} W=m \leq n$. Let $\left(e_{1}^{k}(x), \cdots, e_{m}^{k}(x)\right)$ be a basis in the tangent space $T_{x} W^{k}$ at $x \in W^{k}$ consistent with the orientation of $W^{k}, k \in N$.

We suppose that the sequence of orientation frames $\left(e_{1}^{k}\left(x_{k}\right), \ldots, e_{m}^{k}\left(x_{k}\right)\right)$ of $W^{k}$ at $x_{k}$ converges to $\left(e_{1}(x), \cdots, e_{m}(x)\right)$ for each $x \in W_{0}$, where

$\lim _{k} x_{k}=x \in W_{0}$, while $e_{1}(x), \cdots, e_{m}(x)$ are linearly independent vectors in $R^{n}$.

v). Let a sequence of Riemann volume elements $\lambda_{k}$ on $W^{k}$ (see $\S$ XIII.2 [19]) induce a limit volume element $\lambda$ on $W$, that is,

$\lambda(B \cap W)=\lim _{k \rightarrow \infty}\left(B \cap W^{k}\right)$ for each compact canonical closed subset $B$ in $R^{n}$, consequently,

$\lambda\left(W \backslash W_{0}\right)=0$. We shall consider surface integrals of the second kind, i.e. by the oriented surface $W$ (see (iv)), where each $W_{j}, j=0, \cdots, m-1$ is oriented (see also $\S$ XIII.2.5 [19]).

vi). Let a vector $w \in \operatorname{Int}(U)$ exist so that $U-w$ is convex in $R^{n}$ and let $\partial U$ be connected. Suppose that a boundary $\partial U$ of $U$ satisfies Conditions (i-v) and,

vii) let the orientations of $\partial U^{k}$ and $U^{k}$ be consistent for each $k \in N$ (see Proposition 2 and Definition 3 [19]).

Particularly, the Riemann volume element $\lambda_{k}$ on $\partial U^{k}$ is consistent with the Lebesgue measure on $U^{k}$ induced from $R^{n}$ for each $k$. This induces the measure $\lambda$ on $\partial U$ as in (v).

Also the boundary conditions are imposed:

4) $\left.f(t)\right|_{\partial U}=f_{0}\left(t^{\prime}\right)$,

$\left.\left(\partial^{|q|} f(t) / \partial s_{1}^{q_{1}} \cdots \partial s_{n}^{q_{n}}\right)\right|_{\partial U}=f_{(q)}\left(t^{\prime}\right)$ for $|q| \leq \alpha-1$,

where $s=\left(s_{1}, \cdots, s_{n}\right) \in R^{n}, \quad(q)=\left(q_{1}, \cdots, q_{n}\right)$,

$|q|=q_{1}+\cdots+q_{n}, \quad 0 \leq q_{k} \in Z$ for each $k, \quad t \in \partial U$ is denoted by $t^{\prime}, f_{0}, f_{(q)}$ are given functions. Generally these conditions may be excessive, so one uses some of them or their linear combinations (see (5.1) below). Frequently, the boundary conditions

5) $\left.f(t)\right|_{\partial U}=f_{0}\left(t^{\prime}\right),\left.\quad\left(\partial^{l} f(t) / \partial v^{l}\right)\right|_{\partial U}=f_{l}\left(t^{\prime}\right)$

for $1 \leq l \leq \alpha-1$ are also used, where $v$ denotes a real variable along a unit external normal to the boundary $\partial U$ at a point $t^{\prime} \in \partial U_{0}$. Using partial differentiation in local coordinates on $\partial U$ and (5) one can calculate in principle all other boundary conditions in (4) almost everywhere on $\partial U$.

Suppose that a domain $U_{1}$ and its boundary $\partial U_{1}$ satisfy Conditions $\left(D 1, \mathrm{i}\right.$-vii) and $g_{1}=g \chi_{U_{1}}$ is an original on $R^{n}$ with its support in $U_{1}$. Then any original $g$ on $R^{n}$ gives the original $g \chi_{U_{2}}=: g_{2}$ on $R^{n}$, where $U_{2}=R^{n} \backslash U_{1}$. Therefore, $g_{1}+g_{2}$ is the original on $R^{n}$, when $g_{1}$ and $g_{2}$ are two originals with their supports contained in $U_{1}$ and $U_{2}$ correspondingly. Take now new domain $U$ satisfying Conditions (D1, i-vii) and (D2-D3):

D2) $U \supset U_{1}$ and $\partial U \subset \partial U_{1}$;

D3) if a straight line $\xi$ containing a point $w_{1}$ (see (vi)) intersects $\partial U_{1}$ at two points $y_{1}$ and $y_{2}$, then only one point either $y_{1}$ or $y_{2}$ belongs to $\partial U$, where $w_{1} \in U_{1}, U-w_{1}$ and $U_{1}-w_{1}$ are convex; if $\xi$ intersects $\partial U_{1}$ only at one point, then it intersects $\partial U$ at the same point. That is,

D4) any straight line $\xi$ through the point $w_{1}$ either does not intersect $\partial U$ or intersects the boundary $\partial U$ only at one point.

Take now $g$ with $\operatorname{supp}(g) \subset U$, then $\operatorname{supp}\left(g \chi_{U_{1}}\right) \subset U_{1}$. Therefore, any problem (1) on $U_{1}$ can be considered as the restriction of the problem (1) defined on $U$, satisfying (D1-D4, i-vii). Any solution $f$ of (1) on $U$ with the boundary conditions on $\partial U$ gives the solution as the restriction $\left.f\right|_{U_{1}}$ on $U_{1}$ with the boundary conditions on $\partial U$.

Henceforward, we suppose that the domain $U$ satisfies Conditions $(D 1, D 4, \mathrm{i}$-vii, which are rather mild and natural. In particular, for $Q^{n}$ this means that either $a_{k}=-\infty$ or $b_{k}=+\infty$ for each $k$. Another example is: $U_{1}$ is a ball in $R^{n}$ with the center at zero, $U=U_{1} \cup\left(R^{n} \backslash U_{1 \cdots, 1}\right), w_{1}=0$; or 
$U=U_{1} \cup\left\{t \in R^{n}: t_{n} \geq-\varepsilon\right\}$ with a marked number $0<\varepsilon<1 / 2$. But subsets $\partial U_{(l)}$ in $\partial U$ can also be specified, if the boundary conditions demand it.

The complex field has the natural realization by $2 \times 2$ real matrices so that $i=\left(\begin{array}{c}01 \\ -10\end{array}\right), i^{2}=\left(\begin{array}{c}-10 \\ 01\end{array}\right)$. The quaternion skew field, as it is well-known, can be realized with the help of $2 \times 2$ complex matrices with the generators $I=\left(\begin{array}{l}10 \\ 01\end{array}\right), J=\left(\begin{array}{c}01 \\ -10\end{array}\right), K=\left(\begin{array}{cc}i & 0 \\ 0 & -i\end{array}\right)$, $L=\left(\begin{array}{cc}0 & -i \\ -i & 0\end{array}\right)$, or equivalently by $4 \times 4$ real matrices. Considering matrices with entries in the Cayley-Dickson algebra $A_{v}$ one gets the complexified or quaternionified Cayley-Dickson algebras $\left(A_{v}\right)_{C}$ or $\left(A_{v}\right)_{H}$ with elements $z=a I+b i$ or $z=a I+b J+c K+e L$, where

$a, b, c, e \in A_{v}$, such that each $a \in A_{v}$ commutes with the generators $i, I, J, K$ and $L$. When $r=2, f$ and $g$ have values in $A_{2}=H$ and $2 \leq n \leq 4$ and coefficients of differential operators belong to $A_{2}$, then the multiparameter noncommutative transform operates with the associative case so that

$$
F^{n}(a f)=a F^{n}(f)
$$

for each $a \in H$. The left linearity property $F^{n}(a f)=a F^{n}(f)$ for any $a \in H_{J, K, L}$ is also accomplished for either operators with coefficients in $R$ or $R C_{i}=I R \oplus i R \quad$ or $\quad H_{J, K, L}=I R \oplus J R \oplus K R \oplus L R \quad$ and $f$ with values in $A_{v}$ with $1 \leq n \leq 2^{v}-1$; or vice versa $f$ with values in $C_{i}$ or $H_{J, K, L}$ and coefficients $a$ in $A_{v}$ but with $1 \leq n \leq 4$. Thus all such variants of operator coefficients $a_{j}$ and values of functions $f$ can be treated by the noncommutative transform. Henceforward, we suppose that these variants take place.

We suppose that $g(t)$ is an original function, that is satisfying Conditions 1(1-4). Consider at first the case of constant coefficients $a_{j}$ on a quadrant domain $Q^{n}$. Let $Q^{n}$ be oriented so that $a_{k}=-\infty$ and $b_{k}=+\infty$ for each $k \leq n-\kappa$; either $a_{k}=-\infty$ or $b_{k}=+\infty$ for each $k>n-\kappa$, where $0 \leq \kappa \leq n$ is a marked integer number. If conditions of Theorem 25 are satisfied, then

6) $F^{n}(A[f](t), u ; p ; \zeta)=\sum_{|j| \leq \alpha} a_{j}\left\{\left[R_{e_{1}}(p)\right]^{j_{1}}\left[R_{e_{2}}(p)\right]^{j_{2}} \cdots\left[R_{e_{n}}(p)\right]^{j_{n}} F^{n}\left(f(t) \chi_{Q^{n}}(t), u ; p ; \zeta\right)\right.$

$$
\begin{aligned}
& \sum_{1 \leq(l j) \mid ; m_{k}+q_{k}+h_{k}=j_{k} ; 0 \leq m_{k} ; 0 \leq q_{k} ; h_{k}=\operatorname{sign}\left(l_{k} j_{k}\right) ; q_{k}=0} \text { for } l_{k} j_{k}=0, \text { for each } k=1, \ldots, n ;(l) \in\left\{0,1,2^{n}\right\}^{n} \\
& \left.(-1)^{|(j)| \mid}\left[R_{e_{1}}(p)\right]^{m_{1}}\left[R_{e_{2}}(p)\right]^{m_{2}} \cdots\left[R_{e_{n}}(p)\right]^{m_{n}} F^{n-|h(j)|}\left(\partial^{|q|} f\left(t^{(j)}\right) \chi_{\partial Q_{(l j)}^{n}}\left(t^{(l j)}\right) / \partial t_{1}^{q_{1}} \cdots \partial t_{n}^{q_{n}}, u ; p ; \zeta\right)\right\} \\
& =F^{n}\left(g(t) \chi_{Q^{n}}(t), u ; p ; \zeta\right)
\end{aligned}
$$

for $u(p, t ; \zeta)$ in the $A_{r}$ spherical or $A_{r}$ Cartesian coordinates, where the operators $R_{e_{i}}(p)$ are given by Formulas $25(1.1)$ or $25(1.2)$. Here $(l)$ enumerates faces $\partial Q_{(l)}^{n}$ in $\partial Q_{k-1}^{n}$ for $|h(l)|=k \geq 1$, so that

$\partial Q_{k-1}^{n}=\bigcup_{\mid h(l)=k} Q_{(l)}^{n}, \quad \partial Q_{(l)}^{n} \cap \partial Q_{(m)}^{n}=\varnothing$ for each

$(l) \neq(m)$ in accordance with $\S 25$ and the notation of this section.

Therefore, Equation (6) shows that the boundary conditions are necessary:

$$
\begin{aligned}
& \left.\left(\partial^{|q|} f\left(t^{(l)}\right) / \partial t_{1}^{q_{1}} \cdots \partial t_{n}^{q_{n}}\right)\right|_{\partial Q_{(l)}^{n}} \text { for }|j| \leq \alpha,|(l j)| \geq 1, \\
& a_{j} \neq 0, q_{k}=0 \text { for } l_{k} j_{k}=0, m_{k}+q_{k}+h_{k}=j_{k}, \\
& h_{k}=\operatorname{sign}\left(l_{k} j_{k}\right), k=1, \cdots, n, t^{(l)} \in \partial Q_{(l)}^{n} . \text { But } \\
& \operatorname{dim}_{R} \partial Q^{n}=n-1 \text { for } \partial Q^{n} \neq \varnothing, \text { consequently, } \\
& \left.\left(\partial^{|q|} f\left(t^{(l)}\right) / \partial t_{1}^{q_{1}} \cdots \partial t_{n}^{q_{n}}\right)\right|_{\partial Q_{(l)}^{n}} \text { can be calculated if know } \\
& \left.\left(\partial^{|\beta|} f\left(t^{(l)}\right) / \partial t_{\gamma(1)}^{\beta_{1}} \cdots \partial t_{\gamma(m)}^{\beta_{m}}\right)\right|_{\partial Q_{(l)}^{n}} \text { for }|\beta|=|q|, \text { where }
\end{aligned}
$$

$\beta=\left(\beta_{1}, \cdots, \beta_{m}\right), \quad m=|h(l)|$, a number $\gamma(k)$ corresponds to $l_{\gamma(k)}>0$, since $q_{k}=0$ for $l_{k}=0$ and $q_{k}>0$ only for $l_{k} j_{k}>0$ and $k>n-\kappa$. That is, $t_{\gamma(1)}, \cdots, t_{\gamma(m)}$ are coordinates in $R^{n}$ along unit vectors orthogonal to $\partial Q_{(l)}^{n}$.

Take a sequence $U^{k}$ of sub-domains $U^{k} \subset U^{k+1} \subset U$ for each $k \in N$ so that each $U^{k}=\bigcup_{l=1}^{m(k)} Q_{k, l}^{n}$ is the finite union of quadrants $Q_{k, l}^{n}$, $m(k) \in N$. We choose them so that each two different quadrants may intersect only by their borders, each $U^{k}$ satisfies the same conditions as $U$ and

7) $\lim _{k \rightarrow \infty} \operatorname{dist}\left(U, U^{k}\right)=0$.

Therefore, Equation (6) can be written for more general domain $U$ also.

For $U$ instead of $Q^{n}$ we get a face $\partial U_{(l)}$ instead of $\partial Q_{(l)}^{n}$ and local coordinates $\tau_{\gamma(1)}, \cdots, \tau_{\gamma(m)}$ orthogonal to $\partial U_{(l)}$ instead of $t_{\gamma(1)}, \cdots, t_{\gamma(m)}$ (see Conditions (i-iii) above).

Thus the sufficient boundary conditions are: 
5.1) $\left(\left.\partial^{|\beta|} f\left(\left(t^{(l j)}\right) / \partial \tau_{\gamma(1)}^{\beta_{1}} \cdots \partial \tau_{\gamma(m)}^{\beta_{m}}\right)\right|_{\partial U_{(j)}}=\phi_{\beta,(l j)}\left(t^{(l j)}\right)\right.$

for $|\beta|=|q|$, where $m=|h(l j)|,|j| \leq \alpha,|(l j)| \geq 1$, $a_{j} \neq 0, q_{k}=0$ for $l_{k} j_{k}=0, m_{k}+q_{k}+h_{k}=j_{k}$, $h_{k}=\operatorname{sign}\left(l_{k} j_{k}\right), \quad 0 \leq q_{k} \leq j_{k}-1$ for $k>n-\kappa$; $\phi_{\beta,(l)}\left(t^{(l)}\right)$ are known functions on $\partial U_{(l)}, t^{(l)} \in \partial U_{(l)}$. In the half-space $t_{n} \geq 0$ only

$$
\text { 5.2) } \partial^{\beta} f(t) /\left.\partial t_{n}^{\beta}\right|_{t_{n}=0}
$$

are necessary for $\beta=|q|<\alpha$ and $q$ as above.

Depending on coefficients of the operator $A$ and the domain $U$ some boundary conditions may be dropped, when the corresponding terms vanish in Formula (6). For example, if $A=\partial^{2} / \partial t_{1} \partial t_{2}, U=U_{1,1}, n=2$, then $\partial f /\left.\partial v\right|_{\partial U_{0}}$ is not necessary, only the boundary condition $\left.f\right|_{\partial U}$ is sufficient.

If $U=R^{n}$, then no any boundary condition appears. Mention that

$$
\text { 5.3) } F^{0}(f(a) ; u ; p ; \zeta)=f(a) e^{-u(p, a ; \zeta)},
$$

which happens in (6), when $a=t^{(l)}$ and $|h(l)|=n$.

Conditions in (5.1) are given on disjoint for different (i) submanifolds $\partial U_{(l)}$ in $\partial U$ and partial derivatives are along orthogonal to them coordinates in $R^{n}$, so they are correctly posed.

In $A_{r}$ spherical coordinates due to Corollary 4. Equation (6) with different values of the parameter $\zeta$ gives a system of linear equations relative to unknown functions $S_{(m)} F^{n}(f(t), u ; p ; \zeta)$, from which $F^{n}(f(t), u ; p ; \zeta)$ can be expressed through a family

$$
\left\{S_{(m)} F^{n}(g(t), u ; p ; \zeta) ; S_{(m)} F^{n-|h(l)|}\left(\partial^{|q|} f\left(t^{(l)}\right) \chi_{\partial Q_{(l)}^{n}}\left(t^{(l)}\right) / \partial t_{1}^{q_{1}} \cdots \partial t_{n}^{q_{n}}, u ; p ; \zeta\right):(m) \in Z^{n}\right\}
$$

and polynomials of $p$, where $Z$ denotes the ring of integer numbers, where the corresponding term $F^{n-|h(l)|}$ is zero when $t_{j}^{(l)}= \pm \infty$ for some $j$. In the $A_{r}$ Carte- sian coordinates there are not so well periodicity properties generally, so the family may be infinite. This means that $F^{n}(f(t), u ; p ; \zeta)$ can be expressed in the form:

8) $F^{n}(f(t), u ; p ; \zeta)=\sum_{(m)} P_{(m)}(p) S_{(m)} F^{n}(g(t), u ; p ; \zeta)$

$$
+\sum_{j,(q),(l),(l) \mid \geq 1,(m)} P_{j,(q),(l),(m)}(p) S_{(m)} F^{n-|h(l j)|}\left(\partial^{|q|} f\left(t^{(l j)}\right) \chi_{\partial U_{(j)}}\left(t^{(l j)}\right) / \partial t_{1}^{q_{1}} \cdots \partial t_{n}^{q_{n}}, u ; p ; \zeta\right),
$$

where $P_{(m)}(p)$ and $P_{j,(q),(l),(m)}(p)$ are quotients of polynomials of real variables $p_{0}, p_{1}, \cdots, p_{n}$. The sum in (8) is finite in the $A_{r}$ spherical coordinates and may be infinite in the $A_{r}$ Cartesian coordinates. To the obtained Equation (8) we apply the theorem about the inversion of the noncommutative multiparameter transform. Thus this gives an expression of $f$ through $g$ as a particular solution of the problem given by $(1,2,3.1)$ and it is prescribed by Formulas 6.1(1) and 8.1(1).

For $F^{n}(f ; u ; p ; \zeta)$ Conditions $8(1,2)$ are satisfied, since $P_{(m)}(p)$ and $P_{j,(q),(l),(m)}(p)$ are quotients of polynomials with real, complex or quaternion coefficients and real variables, also $G^{n}$ and $F^{n-|h(l)|}$ terms on the right of (6) satisfy them. Thus we have demonstrated the theorem.

\subsubsection{Theorem}

Suppose that $F^{n}(f ; u ; p ; \zeta)$ given by the right side of (8) satisfies Conditions 8(3). Then Problem $(1,2,3.1)$ has a solution in the class of original functions, when $g$ and $\phi_{\beta,(l)}$ are originals, or in the class of generalized functions, when $g$ and $\phi_{\beta,(l)}$ are generalized functions.

Mention, that a general solution of $(1,2)$ is the sum of its particular solution and a general solution of the homogeneous problem $A f=0$. If $\phi_{\beta,(l)}=\phi_{\beta,(l)}^{1}+\phi_{\beta,(l)}^{2}$, $g=g_{1}+g_{2}, f=f_{1}+f_{2}, A f_{j}=g_{j}$ and $f_{j}$ on $\partial U_{j}$ satisfies (5.1) with $\phi_{\beta,(l)}^{j}, j=1,2$, then $A f=g$ and $f$ on $\partial U$ satisfies Conditions (5.1) with $\phi_{\beta,(l)}$.

\subsubsection{Example}

We take the partial differential operator of the second order

$$
A=\sum_{h, m=1}^{n} a_{h, m} \partial^{2} / \partial \tau_{h} \partial \tau_{m}+\sum_{h=1}^{n} \alpha_{h} \partial / \partial \tau_{h}+\omega,
$$

where the quadratic form $a(\tau):=\sum_{h, m} a_{h, m} \tau_{h} \tau_{m}$ is nondegenerate and is not always negative, because otherwise we can consider $-A$. Suppose that $a_{h, m}=a_{m, h} \in R$, $\alpha_{h}, \tau_{h} \in R$ for each $h, m=1, \cdots, n, \omega \in A_{3}$. Then we reduce this form $a(\tau)$ by an invertible $R$ linear operator $C$ to the sum of squares. Thus

$$
\text { 9) } A=\sum_{h=1}^{n} a_{h} \partial^{2} / \partial t_{h}^{2}+\sum_{h=1}^{n} \beta_{h} \partial / \partial t_{h}+\omega,
$$

where $\left(t_{1}, \cdots, t_{n}\right)=\left(\tau_{1}, \cdots, \tau_{n}\right) C$ with real $a_{h}$ and $\beta_{h}$ for each $h$. If coefficients of $A$ are constant, using a multiplier of the type $\exp \left(\sum_{h} \varepsilon_{h} s_{h}\right)$ it is possible to reduce this equation to the case so that if $a_{h} \neq 0$, then $\beta_{h}=0$ (see $\S 3$, Chapter 4 in [20]). Then we can simplify the operator with the help of a linear trans- 
formation of coordinates and consider that only $\beta_{n}$ may be non-zero if $a_{n}=0$. For $A$ with constant coefficients as it is well-known from algebra one can choose a constant invertible real matrix $\left(c_{h, m}\right)_{h, m=1, \cdots, k}$ corresponding to $C$ so that $a_{h}=1$ for $h \leq k_{+}$and $a_{h}=-1$ for $h>k_{+}$, where $0<k_{+} \leq n$. For $k_{+}=n$ and $\beta=0$ the operator is elliptic, for $k_{+}=n-1$ with $a_{n}=0$ and $\beta_{n} \neq 0$ the operator is parabolic, for $0<k_{+}<n$ and $\beta=0$ the operator is hyperbolic. Then Equation (6) simplifies:

$$
\text { 10) } \begin{aligned}
& F^{n}(A[f](t), u ; p ; \zeta)=\sum_{h=1}^{n} a_{h}\left\{\left[R_{e_{h}}(p)\right]^{2} F^{n}\left(f(t) \chi_{Q^{n}}(t), u ; p ; \zeta\right)\right. \\
+ & \sum_{l_{h} \in\{1,2\} ;(l)=l_{h} e_{h}}(-1)^{l_{h}}\left[F^{n-1}\left(\partial f\left(t^{(l)}\right) \chi_{\partial Q_{(l)}^{n}}\left(t^{(l)}\right) / \partial t_{h}, u ; p ; \zeta\right)+\left[R_{e_{h}}(p)\right]\left(F^{n-1}\left(f\left(t^{(l)}\right) \chi_{\partial Q_{(l)}^{n}}\left(t^{(l)}\right), u ; p ; \zeta\right)\right]\right\} \\
+ & \beta_{n}\left\{F^{n-1 ; t^{n, 2}}\left(f\left(t^{n, 2}\right) \chi_{\partial Q_{2 e_{n}}^{n}}\left(t^{n, 2}\right), u ; p ; \zeta\right)-F^{n-1 ; t^{n, 1}}\left(f\left(t^{n, 1}\right) \chi_{\partial Q_{e_{n}^{n}}}\left(t^{n, 1}\right), u ; p ; \zeta\right)\right. \\
+ & {\left.\left[R_{e_{n}}(p)\right] F^{n}\left(f(t) \chi_{Q^{n}}(t), u ; p ; \zeta\right)\right\}+\omega F^{n}\left(f(t) \chi_{Q^{n}}(t), u ; p ; \zeta\right)=F^{n}(g(t), u ; p ; \zeta) }
\end{aligned}
$$

in the $A_{r}$ spherical or $A_{r}$ Cartesian coordinates, where $e_{h}=(0, \cdots, 0,1,0, \cdots, 0) \in R^{n}$ with 1 on the $h$-th place, $S_{0}=I$ is the unit operator, the operators $R_{e_{h}}(p)$ are given by Formulas 25(1.1) or 25(12) respectively.

We denote by $\delta_{S}(x)$ the delta function of a continuous piecewise differentiable manifold $S$ in $R^{n}$ satisfying conditions (i-vi) so that

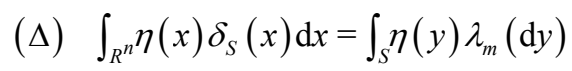

for a continuous integrable function $\eta(x)$ on $R^{n}$, where $\operatorname{dim}(S)=m<n, \lambda_{m}(\mathrm{~d} y)$ denotes a volume element on the $m$ dimensional surface $S$ (see Condition (v) above). Thus we can consider a non-commutative multiparameter transform on $\partial U$ for an original $f$ on $U$ given by the formula:

$$
\text { 11) } \begin{aligned}
& F_{\partial U}^{n-1 ; t^{\prime}}\left(f\left(t^{\prime}\right) \chi_{\partial U}\left(t^{\prime}\right), u ; p ; \zeta\right) . \\
= & F^{n ; t}\left(f(t) \delta_{\partial U}(t), u ; p ; \zeta\right)
\end{aligned}
$$

Therefore, terms like $F^{n-1}$ in (10) correspond to the boundary $\partial Q^{n}$. They can be simplified:

$$
\text { 12) } \begin{aligned}
& \beta_{n}\left\{F^{n-1 ; t^{n, 2}}\left(f\left(t^{n, 2}\right) \chi_{\partial Q_{2 e_{n}}^{n}}(t), u ; p ; \zeta\right)-F^{n-1 ; t^{n, 1}}\left(f\left(t^{n, 1}\right) \chi_{\partial Q_{e_{n}}^{n}}(t), u ; p ; \zeta\right)\right\} \\
= & F_{\partial Q^{n}}^{n-1 ; t^{\prime}}\left(\beta\left(t^{\prime}\right) f\left(t^{\prime}\right) \chi_{\partial Q^{n}}\left(t^{\prime}\right), u ; p ; \zeta\right),
\end{aligned}
$$

where $\beta\left(t^{\prime}\right)$ is a piecewise constant function on $\partial Q^{n}$ equal to $\beta_{n}$ on the corresponding faces of $Q^{n}$ orthogonal to $e_{n}$ given by condition: either $t_{n}=a_{n}$ or $t_{n}=b_{n} ; \beta\left(t^{\prime}\right)=0$ is zero otherwise.

If $a_{k}=-\infty$ or $b_{k}=+\infty$, then the corresponding term disappears. If $R^{n}$ embed into $A_{r}$ with $2^{r-1} \leq n \leq 2^{r}-1$

as $R i_{1} \oplus \cdots \oplus R i_{n}$, then this induces the corresponding embedding $\Theta$ of $Q^{n}$ or $U$ into $A_{r}$. This permits to make further simplification:

$$
\text { 12.1) } \begin{aligned}
& \sum_{h=1}^{n} a_{h}\left\{\sum_{l_{h} \in\{1,2\} ;(l)=l_{h} e_{h}}(-1)^{l_{h}}\left[\left[R_{e_{h}}(p)\right] F^{n-1}\left(f\left(t^{(l)}\right) \chi_{\partial Q_{(l)}^{n}}\left(t^{(l)}\right), u ; p ; \zeta\right)+F^{n-1}\left(\partial f\left(t^{(l)}\right) \chi_{\partial Q_{(l)}^{n}}\left(t^{(l)}\right) / \partial t_{h}, u ; p ; \zeta\right)\right]\right\} \\
& =F_{\partial Q^{n}}^{n-1}\left(a\left(t^{\prime}\right)\left(\partial f\left(t^{\prime}\right) \chi_{\partial Q_{0}^{n}}\left(t^{\prime}\right) / \partial v\right), u\left(p, t^{\prime} ; \zeta\right) ; p ; \zeta\right)+F_{\partial Q^{n}}^{n-1}\left(P\left(t^{\prime}\right) f\left(t^{\prime}\right) \chi_{\partial Q_{0}^{n}}\left(t^{\prime}\right), u ; p ; \zeta\right),
\end{aligned}
$$

where $v=v\left(t^{\prime}\right)$ denotes a real coordinate along an external unit normal $M\left(t^{\prime}\right)$ to $\Theta(\partial U)$ at $\Theta\left(t^{\prime}\right)$, so that $M\left(t^{\prime}\right)$ is a purely imaginary Cayley-Dickson number, $a\left(t^{\prime}\right)$ is a piecewise constant function equal to $a_{h}$ for the corresponding $t^{\prime}$ in the face $\partial Q_{l_{h} e_{h}}^{n}$ with $l_{h}>0$; $P\left(t^{\prime}, p\right):=P\left(t^{\prime}\right):=R_{e_{h}}(p)$ for $t^{\prime} \in \partial Q_{l_{h} e_{h}}^{h}, \quad h=1, \cdots, n$, since $\sin (\psi+\pi)=-\sin (\psi)$ and $\cos (\psi+\pi)=-\cos (\psi)$ for each $\psi \in R$. Certainly the operator-valued function $P\left(t^{\prime}\right)$ has a piecewise continuous extension $P(t)$ on $Q^{n}$. That is

$$
\text { 13) } \begin{aligned}
& F_{\partial U}^{n-1}\left(\xi\left(t^{\prime}\right) f\left(t^{\prime}\right) \chi_{\partial U}\left(t^{\prime}\right), u\left(p, t^{\prime} ; \zeta\right) ; p ; \zeta\right) \\
&:=\int_{R^{n}} \xi(t) f(t) \delta_{\partial U}(t) \exp \{-u(p, t ; \zeta)\} \mathrm{d} t
\end{aligned}
$$


for an integrable operator-valued function $\xi(t)$ so that $[\xi(t) f(t)]$ is an original on $U$ whenever this in- tegral exists. For example, when $\xi$ is a linear combination of shift operators $S_{(m)}$ with coefficients $\varepsilon_{(m)}(t, p)$ such that each $\varepsilon_{(m)}(t, p)$ as a function by $t \in U$ for each $p \in W$ and $f(t)$ are originals or $f$ and $g$ are generalized functions. For two quadrants $Q_{m, l}$ and $Q_{m, k}$ intersecting by a common face $\Upsilon$ external normals to it for these quadrants have opposite directions. Thus the corresponding integrals in $F_{\partial Q_{m, l}}^{n-1}$ and $F_{\partial Q_{m, k}}^{n-1}$ restricted on $\Upsilon$ summands cancel in $F_{\partial\left(Q_{m, l}^{n-1} \cup Q_{m, k}\right)}^{n}$.

Using Conditions (iv-vii) and the sequence $U^{m}$ and quadrants $Q_{m, l}^{n}$ outlined above we get for a boundary problem on $U$ instead of $Q^{n}$ the following equation:

$$
\text { 14) } \begin{aligned}
& F^{n}(A[f](t), u ; p ; \zeta)=\left\{\sum_{h=1}^{n} a_{h}\left[R_{e_{h}}(p)\right]^{2} F^{n}\left(f(t) \chi_{U}(t), u ; p ; \zeta\right)\right\} \\
+ & \left.\left\{F_{\partial U}^{n-1}\left(\left[\beta\left(t^{\prime}\right)\right)+P\left(t^{\prime}, p\right)\right] f\left(t^{\prime}\right) \chi_{\partial U_{0}}\left(t^{\prime}\right), u ; p ; \zeta\right)+F_{\partial U}^{n-1}\left(a\left(t^{\prime}\right)\left(\partial f\left(t^{\prime}\right) \chi_{\partial U_{0}}\left(t^{\prime}\right) / \partial v\right), u ; p ; \zeta\right)\right\} \\
& F^{n}\left(\beta_{n}\left[R_{n}(p)\right] f(t) \chi_{U}(t), u ; p ; \zeta\right)+\omega F^{n}\left(f(t) \chi_{U}(t), u ; p ; \zeta\right)=F^{n}(g(t), u ; p ; \zeta),
\end{aligned}
$$

where $P\left(t^{\prime}, p\right):=P\left(t^{\prime}\right):=\sum_{h=1}^{n} a_{h}\left[R_{h}(p)\right]\left(\partial v / \partial t_{h}\right)$

for each $t^{\prime} \in \partial U_{0}$ (see also Stokes' formula in $\S$ XIII. 3.4 [19] and Formulas $(14.2,14.3)$ below). Particularly, for the quadrant domain $Q^{n}$ we have $a(t)=a_{h}$ for $t \in \partial Q_{h_{h} e_{h}}^{n}$ with $l_{h}>0, \beta(t)=\beta_{n}$ for $t \in \partial Q_{l_{n} e_{n}}^{n}$ with $l_{n}>0$ and zero otherwise.

The boundary conditions are:

$$
\text { 14.1) }\left.f(t)\right|_{\partial U_{0}}=\left.\phi(t)\right|_{\partial U_{0}},\left.(\partial f(t) / \partial v)\right|_{\partial U_{0}}=\left.\phi_{1}(t)\right|_{\partial U_{0}} \text {. }
$$

The functions $a(t)$ and $\beta(t)$ can be calculated from $\left\{a_{h}: h\right\}$ and $\beta_{n}$ almost everywhere on $\partial U$ with the help of change of variables from $\left(t_{1}, \cdots, t_{n}\right)$ to $\left(y_{1}, \cdots, y_{n-1}, y_{n}\right)$, where $\left(y_{1}, \cdots, y_{n}\right)$ are local coordinates in $\partial U_{0}$ in a neighborhood of a point $t^{\prime} \in \partial U_{0}$, $y_{n}=v$, since $\partial U_{0}$ is of class $C^{1}$. Consider the differential form

$$
\sum_{h=1}^{n}(-1)^{n-h} a_{h} \mathrm{~d} t_{1} \wedge \cdots \wedge \widehat{\mathrm{d} t_{h}} \wedge \cdots \wedge \mathrm{d} t_{n}=a \mathrm{~d} y_{1} \wedge \cdots \wedge \mathrm{d} y_{n-1}
$$

and its external product with $\mathrm{d} v=\sum_{h=1}^{n}\left(\partial v / \partial t_{h}\right) \mathrm{d} t_{h}$, then

14.2) $\left.a(t)\right|_{\partial U_{0}}=\left.\sum_{h=1}^{n} a_{h}\left(\partial v / \partial t_{h}\right)\right|_{\partial U_{0}}$ and

14.3) $\left.\beta(t)\right|_{\partial U_{0}}=\left.\beta_{n} \chi_{U_{e_{n}} \cup U_{2 e_{n}}}\left(\partial v / \partial t_{n}\right)\right|_{\partial U_{0}}$.

This is sufficient for the calculation of $F_{\partial U}^{n-1}$.

\subsubsection{Inversion Procedure in the $A_{r}$ Spherical Coordinates}

When boundary conditions 28(3.1) are specified, this Equation 28(6) can be resolved relative to

$F^{n}\left(f(t) \chi_{U}(t), u(p, t ; \zeta) ; p ; \zeta\right)$, particularly, for Equations 28.2(14,14.1) also. The operators $S_{e_{j}}$ and $T_{j}$ of $\S 12$ have the periodicity properties:

$$
\begin{gathered}
S_{e_{j}}^{4+k} F(p ; \zeta)=S_{e_{j}}^{k} F(p ; \zeta) \text { and } \\
T_{j}^{4+k} F(p ; \zeta)=T_{j}^{k} F(p ; \zeta), \\
S_{e_{1}}^{2+k} F(p ; \zeta)=-S_{e_{1}}^{k} F(p ; \zeta) \text { and } \\
T_{1}^{2+k} F(p ; \zeta)=-T_{1}^{k} F(p ; \zeta)
\end{gathered}
$$

for each positive integer number $k$ and $1 \leq j \leq 2^{r}-1$. We put

$$
\text { 6.1) } F_{j}(p ; \zeta):=\left(S_{e_{j}}^{4}-S_{e_{j+1}}^{4}\right) F(p ; \zeta)
$$

for any $1 \leq j \leq 2^{r}-2$,

$$
\text { 6.2) } F_{2^{r}-1}(p ; \zeta):=S_{e^{2^{r}-1}}^{4} F(p ; \zeta) \text {. }
$$

Then from Formula 28(6) we get the following equations:

$$
\begin{aligned}
& \text { 6.3) }\left.\sum_{|j| \leq \alpha} a_{j}\left\{\left[p_{0}+p_{1} T_{1}\right]^{j_{1}}\left[p_{0}+p_{1} T_{1}+p_{2} T_{2}\right]^{j_{2}} \ldots\left[p_{0}+p_{1} T_{1}+\ldots+p_{n} T_{n}\right]^{j_{n}}\right\}\right|_{p_{b}=0 \forall b>w} F_{w}(p ; \zeta) \\
& =\left\{-\sum_{|j| \leq \alpha} a_{j} \sum_{1 \leq(l j) ; m_{k}+q_{k}+h_{k}=j_{k} ; 0 \leq m_{k} ; 0 \leq q_{k} ; h_{k}=\operatorname{sign}\left(l_{k} j_{k}\right) ; q_{k}=0} \text { for } l_{k} j_{k}=0 \text {, for each } k=1, \cdots, n ;(l) \in\{0,1,2\}^{n}\right. \\
& \left.(-1)^{|(j)|}\left\{\left[p_{0}+p_{1} T_{1}\right]^{m_{1}}\left[p_{0}+p_{1} T_{1}+p_{2} T_{2}\right]^{m_{2}} \cdots\left[p_{0}+p_{1} T_{1}+\cdots+p_{n} T_{n}\right]^{m_{n}}\right\}\right|_{p_{b}=0 \forall b>w}
\end{aligned}
$$

for each $w=1, \cdots, n$, where

$$
F(p ; \zeta)=F^{n}\left(f(t) \chi_{Q^{n}}(t), u ; p ; \zeta\right) \text { and }
$$

$$
G(p ; \zeta)=F^{n}\left(g(t) \chi_{Q^{n}}(t), u ; p ; \zeta\right) .
$$

These equations are resolved for each $w=1, \cdots, n$ as it 
is indicated below. Taking the sum one gets the result

6.4) $F(p ; \zeta)=F_{1}(p ; \zeta)+\cdots+F_{n}(p ; \zeta)$,

Since

$$
\begin{aligned}
& \left\{\left[\sum_{j=1}^{2^{r}-2}\left(S_{e_{j}}^{4}-S_{e_{j+1}}^{4}\right)\right]+S_{e_{2^{r}-1}^{4}}^{4}\right\} e^{-u(p, t ; \zeta)} . \\
& =S_{e_{1}}^{4} e^{-u(p, t ; \zeta)}=e^{-u(p, t ; \zeta)}
\end{aligned}
$$

The analogous procedure is for Equation (14) with the domain $U$ instead of $Q^{n}$.

From Equation (6.3) or (14) we get the linear equation:

$$
\text { 15) } \sum_{(l)} \psi_{(l)} x_{(l)}=\phi
$$

where $\phi$ is the known function and depends on the parameter $\zeta, \psi_{(l)}$ are known coefficients depending on $p, x_{(l)}$ are indeterminates and may depend on $\zeta$, $l_{1}=0,1$ for $h=1$, so that $x_{(l)+2 e_{1}}=-x_{(l)} ; l_{h}=0,1,2,3$ for $h>1$, where $x_{(l)+4 e_{h}}=x_{(l)}$ for each $h>1$ in accordance with Corollary 4.1, $(l)=\left(l_{1}, \cdots, l_{n}\right)$.

Acting on both sides of (6.3) or (14) with the shift operators $T_{(m)}$ (see Formula 25(SO)), where $m_{1}=0,1$, $m_{h}=0,1,2,3$ for each $h>1$, we get from (15) a system of $2^{1+2(k-1)}$ linear equations with the known functions $\phi_{(m)}:=T_{(m)} \phi$ instead of $\phi, \phi$ :

15.1) $\sum_{(l)} \psi_{(l)} T_{(m)} x_{(l)}=\phi_{(m)}$ for each $(m)$.

Each such shift of $\zeta$ left coefficients $\psi_{(l)}$ intact and $x_{(l)+(m)}=(-1)^{\eta} x_{\left(l^{\prime}\right)}$ with $l_{1}^{\prime}=l_{1}+m_{1}(\bmod 2)$, $l_{h}^{\prime}=l_{h}+m_{h}(\bmod 4)$ for each $h>1$, where $\eta=1$ for $l_{1}+m_{1}-l_{1}^{\prime}=2, \eta=2$ otherwise. This system can be reduced, when a minimal additive group

$$
G:=\left\{(l): l_{1}(\bmod 2), l_{j}(\bmod 4) \forall 2 \leq j \leq k ;\right.
$$

generated by all $(l)$ with non-zero

coefficients in Equation (15)\}

is a proper subgroup of $g_{2} \times g_{4}^{k-1}$, where $g_{h}:=Z /(h Z)$ denotes the finite additive group for $0<h \in Z$. Generally the obtained system is non-degenerate for $\lambda_{n+1}$ almost all $p=\left(p_{0}, \cdots, p_{n}\right) \in R^{n+1}$ or in $W$, where $\lambda_{n+1}$ denotes the Lebesgue measure on the real space $R^{n+1}$.

We consider the non-degenerate operator $A$ with real, complex $C_{i}$ or quaternion $H_{J, K, L}$ coefficients. Certainly in the real and complex cases at each point $p$, where its determinate $\Delta=\Delta(p)$ is non-zero, a solution can be found by the Cramer's rule.

Generally, the system can be solved by the following algorithm. We can group variables by $l_{1}, l_{2}, \cdots, l_{k}$. For a given $l_{2}, \cdots, l_{h}$ and $l_{1}=0,1$ subtracting all other terms from both sides of (15) after an action of $T_{(m)}$ with $m_{1}=0,1$ and marked $m_{h}$ for each $h>1$ we get the system of the form

16) $\alpha x_{1}+\beta x_{2}=b_{1},-\beta x_{1}+\alpha x_{2}=b_{2}$, which generally has a unique solution for $\lambda_{n+1}$ almost all $p$ :

$$
\text { 17) } \begin{aligned}
x_{1} & =\left(\alpha\left(\alpha^{2}+\beta^{2}\right)^{-1}\right) b_{1}-\left(\beta\left(\alpha^{2}+\beta^{2}\right)^{-1}\right) b_{2} ; \\
x_{2} & =\left(\alpha\left(\alpha^{2}+\beta^{2}\right)^{-1}\right) b_{2}+\left(\beta\left(\alpha^{2}+\beta^{2}\right)^{-1}\right) b_{1},
\end{aligned}
$$

where $b_{1}, b_{2} \in A_{r}$, for a given set $\left(m_{2}, \cdots m_{n}\right)$.

When $l_{h}$ are specified for each $1 \leq h \leq k$ with $h \neq h_{0}$, where $1<h_{0} \leq k$, then the system is of the type:

18) $a x_{1}+b x_{2}+c x_{3}+d x_{4}=b_{1}$,

$$
\begin{aligned}
& d x_{1}+a x_{2}+b x_{3}+c x_{4}=b_{2}, \\
& c x_{1}+d x_{2}+a x_{3}+b x_{4}=b_{3}, \\
& b x_{1}+c x_{2}+d x_{3}+a x_{4}=b_{4},
\end{aligned}
$$

where $a, b, c, d \in R$ or $C_{i}$ or $H_{J, K, L}$, while

$b_{1}, b_{2}, b_{3}, b_{4} \in A_{r}$. In the latter case of $H_{J, K, L}$ it can be solved by the Gauss' exclusion algorithm. In the first two cases of $R$ or $C_{i}$ the solution is:

19) $x_{j}=\Delta_{j} / \Delta$, where

$$
\begin{aligned}
& \Delta=a \xi_{1}-d \xi_{2}+c \xi_{3}-b \xi_{4}, \\
& \Delta_{1}=b_{1} \xi_{1}-b_{2} \xi_{2}+b_{3} \xi_{3}-b_{4} \xi_{4}, \\
& \Delta_{2}=-b_{1} \xi_{4}+b_{2} \xi_{1}-b_{3} \xi_{2}+b_{4} \xi_{3}, \\
& \Delta_{3}=b_{1} \xi_{3}-b_{2} \xi_{4}+b_{3} \xi_{1}-b_{4} \xi_{2}, \\
& \Delta_{4}=-b_{1} \xi_{2}+b_{2} \xi_{3}-b_{3} \xi_{4}+b_{4} \xi_{1}, \\
& \xi_{1}=a^{3}+b^{2} c+c d^{2}-a c^{2}-2 a b d, \\
& \xi_{2}=a^{2} b+b c^{2}+d^{3}-b^{2} d-2 a c d, \\
& \xi_{3}=a b^{2}+c^{3}+a d^{2}-a^{2} c-2 b c d, \\
& \xi_{4}=a^{2} d+b^{3}+c^{2} d-b d^{2}-2 a b c .
\end{aligned}
$$

Thus on each step either two or four indeterminates are calculated and substituted into the initial linear algebraic system that gives new linear algebraic system with a number of indeterminates less on two or four respectively. May be pairwise resolution on each step is simpler, because the denominator of the type $\left(\alpha^{2}+\beta^{2}\right)$ should be $\lambda_{2^{r}}$ almost everywhere by $p \in A_{r}$ positive (see (6), (14) above). This algorithm acts analogously to the Gauss' algorithm. Finally the last two or four indeterminates remain and they are found with the help of Formulas either (17) or (19) respectively. When for a marked $h$ in (6) or $(14)$ all $l_{h}=0(\bmod 2)$ (remains only $x_{1}$ for $h=1$, or remain $x_{1}$ and $x_{3}$ for $h>1$ ) or for some $h>1$ all $l_{h}=0(\bmod 4)$ (remains only $\left.x_{1}\right)$ a system of linear equations as in $(13,13.1)$ simplifies.

Thus a solution of the type prescribed by (8) generally $\lambda_{n+1}$ almost everywhere by $p \in W$ exists, where $W$ is a domain

$$
W=\left\{p \in A_{r}: a_{1}<\operatorname{Re}(p)<a_{-1}, p_{j}=0 \forall j>n\right\}
$$

of convergence of the noncommutative multiparameter 
transform, when it is non-void, $2^{r-1} \leq n \leq 2^{r}-1$, $\operatorname{Re}(p)=p_{0}, \quad p=p_{0} i_{0}+\cdots+p_{n} i_{n}$.

This domain $W$ is caused by properties of $g$ and initial conditions on $\partial U$ and by the domain $U$ also. Generally $U$ is worthwhile to choose with its interior Int $(U)$ non-intersecting with a characteristic surface $\phi\left(x_{1}, \cdots, x_{n}\right)=0$, i.e. at each point $x$ of it the condition is satisfied

$$
(C S) \sum_{|j|=\alpha} a_{j}(t(x))\left(\partial \phi / \partial x_{1}\right)^{j_{1}} \cdots\left(\partial \phi / \partial x_{n}\right)^{j_{n}}=0
$$

and at least one of the partial derivatives $\left(\partial \phi / \partial x_{k}\right) \neq 0$ is non-zero.

In particular, the boundary problem may be with the right side $g=\varsigma f$ in $(2,2.1,14)$, where $\varsigma$ is a real or complex $C_{i}$ or quaternion $H_{J, K, L}$ multiplier, when boundary conditions are non-trivial. In the space either
$D\left(R^{n}, A_{r}\right)$ or $B\left(R^{n}, A_{r}\right)$ (see $\S 19$ ) a partial differential problem simplifies, because all boundary terms disappear. If $f \in B\left(R^{n}, A_{r}\right)$, then $\left\{p \in A_{r}: \operatorname{Re}(p) \geq 0\right\} \subset W_{f}$. For $f \in D\left(R^{n}, A_{r}\right)$ certainly $W_{f}=A_{r}$ (see also $\S 9$ ).

\subsubsection{Examples}

Take partial differential equations of the fourth order. In this subsection the noncommutative multiparameter transforms in $A_{r}$ spherical coordinates are considered. For

$$
\text { 20) } A=\partial^{3} / \partial s_{1}^{3}+\sum_{j=2}^{n} \gamma_{j} \partial^{4} / \partial s_{j}^{4}
$$

with constants $\gamma_{j} \in H_{J, K, L} \backslash\{0\}$ on the space either $D\left(R^{n}, A_{r}\right)$ or $B\left(R^{n}, A_{r}\right)$, where $n \geq 2$, Equation (6) takes the form:

$$
\text { 21) } \begin{aligned}
& F^{n}(A[f](t), u ; p ; \zeta)=\left\{p_{0}\left(p_{0}^{2}+3\left(p_{1} S_{e_{1}}\right)^{2}\right)+\sum_{j=2}^{n} \gamma_{j}\left(p_{j} S_{e_{j}}\right)^{4}\right\} F^{n}(f(t), u ; p ; \zeta) \\
& +p_{1}\left(3 p_{0}^{2}+\left(p_{1} S_{e_{1}}\right)^{2}\right) S_{e_{1}} F^{n}(f(t), u ; p ; \zeta)=F^{n}(g(t), u ; p ; \zeta)
\end{aligned}
$$

due to Corollary 4.1. In accordance with $(16,17)$ we get:

$$
\text { 22) } \begin{aligned}
& F_{w}(p ; \zeta)=\left(\alpha\left(\alpha^{2}+\beta^{2}\right)^{-1}\right) G_{w}(p ; \zeta) \\
& -\left(\beta\left(\alpha^{2}+\beta^{2}\right)^{-1}\right) T_{1} G_{w}(p ; \zeta)
\end{aligned}
$$

for each $w=1, \cdots, n$,

where

$$
\begin{aligned}
& \alpha_{w}=\alpha=\left.\left[p_{0}\left(p_{0}^{2}-3 p_{1}^{2}\right)+\sum_{j=2}^{n} \gamma_{j} p_{j}^{4}\right]\right|_{p_{b}=0 \forall b>w}, \\
& \beta_{w}=\beta=\left.p_{1}\left(3 p_{0}^{2}-p_{1}^{2}\right)\right|_{p_{b}=0 \forall b>w} .
\end{aligned}
$$

From Theorem 6, Corollary 6.1 and Remarks 24 we infer that:

$$
\begin{aligned}
f(t)= & (2 \pi)^{-n} \int_{R^{n}} F(a+p ; \zeta) \\
& \exp \{u(p, t ; \zeta)\} \mathrm{d} p_{1} \cdots \mathrm{d} p_{n}
\end{aligned}
$$

supposing that the conditions of Theorem 6 and Corollary 6.1 are satisfied, where $F(p ; \zeta)=F^{n}(f(t), u ; p ; \zeta)$.

If on the space either $D\left(R^{k}, A_{r}\right)$ or $B\left(R^{k}, A_{r}\right)$ an operator is as follows:

24) $A=\partial^{4} / \partial s_{1}^{2} \partial s_{2}^{2}+\sum_{j=3}^{n} \gamma_{j} \partial^{4} / \partial s_{j}^{4}$, where $\gamma_{j} \in H_{J, K, L} \backslash\{0\}$, where $n \geq 3$, then (6) reads as:

25) $F^{n}(A f(t), u ; p ; \zeta)=p_{2}^{2}\left(p_{0}^{2}+\left(p_{1} S_{e_{1}}\right)^{2}\right) S_{e_{2}}^{2} F^{n}(f(t), u ; p ; \zeta)$

$$
+2 p_{0} p_{1} p_{2}^{2} S_{e_{1}} S_{e_{2}}^{2} F^{n}\left((f(t)), u ; p ; \zeta+\sum_{j=3}^{n} \gamma_{j}\left(p_{j} S_{e_{j}}\right)^{4} F^{n}(f(t)), u ; p ; \zeta\right)=F^{n}(g(t), u ; p ; \zeta)
$$

If on the same spaces an operator is:

26) $A=\partial^{3} / \partial s_{1} \partial s_{2}^{2}+\sum_{j=3}^{n} \gamma_{j} \partial^{4} / \partial s_{j}^{4}$, where $n \geq 3$, then (6) takes the form:

27) $F^{n}(A f(t), u ; p ; \zeta)=p_{0} p_{2}^{2} S_{e_{2}}^{2} F^{n}(f(t), u ; p ; \zeta)$

$$
+p_{1} p_{2}^{2} S_{e_{1}} S_{e_{2}}^{2} F^{n}(f(t), u ; p ; \zeta)+\sum_{j=3}^{n} \gamma_{j}\left(p_{j} S_{e_{j}}\right)^{4} F^{n}(f(t), u ; p ; \zeta)=F^{n}(g(t), u ; p ; \zeta) \text {. }
$$

To find $F^{n}(f(t), u ; p ; \zeta)$ in (23) or (27) after an action of suitable shift operators $T_{(0,2,0, \cdots, 0)}, T_{(1,0, \cdots, 0)}$ and

$T_{(1,2,0, \cdots, 0)}$ we get the system of linear algebraic equations:

28) $a x_{1}+b x_{3}+c x_{4}=b_{1}, \quad b x_{1}+c x_{2}+a x_{3}=b_{2}, a x_{2}-c x_{3}+b x_{4}=b_{3},-c x_{1}+b x_{2}+a x_{4}=b_{4}$ 
with coefficients $a, b$ and $c$, and Cayley-Dickson numbers on the right side $b_{1}, \cdots, b_{4} \in A_{r}$, where

$$
\begin{gathered}
x_{1}=F_{w}(p ; \zeta), \quad x_{2}=T_{1} F_{w}(p ; \zeta), \quad x_{3}=T_{2}^{2} F_{w}(p ; \zeta), \quad x_{4}=T_{1} T_{2}^{2} F_{w}(p ; \zeta), \\
b_{1}=G_{w}(p ; \zeta)=\left(F^{n}(g(t), u ; p ; \zeta)\right)_{w}, \quad b_{2}=T_{2}^{2} G_{w}(p ; \zeta), \quad b_{3}=T_{1} G_{w}(p ; \zeta), b_{4}=T_{1} T_{2}^{2} G_{w}(p ; \zeta) .
\end{gathered}
$$

Coefficients are:

$$
a_{w}=a=\left.\left[\sum_{j=3}^{n} \gamma_{j} p_{j}^{4}\right]\right|_{p_{b}=0 \forall b>w} \in H_{J, K, L}, \quad b_{w}=b=p_{2}^{2}\left(p_{0}^{2}-p_{1}^{2}\right) \in R, \quad c_{w}=c=\left.2 p_{0} p_{1} p_{2}^{2}\right|_{p_{b}=0 \forall b>w} \in R,
$$

for $A$ given by (24);

$$
a_{w}=a=\left.\left[\sum_{j=3}^{n} \gamma_{j} p_{j}^{4}\right]\right|_{p_{b}=0 \forall b>w} \in H_{J, K, L}, \quad b_{w}=b=\left.p_{0} p_{2}^{2}\right|_{p_{b}=0 \forall b>w} \in R, \quad c_{w}=c=\left.p_{1} p_{2}^{2}\right|_{p_{b}=0 \forall b>w} \in R
$$

for $A$ given by (26), $w=1, \cdots, n$. If $a=0$ the system reduces to two systems with two indeterminates $\left(x_{1}, x_{2}\right)$ and $\left(x_{3}, x_{4}\right)$ of the type described by (16) with so- lutions given by Formulas (17). It is seen that these coefficients are non-zero $\lambda_{n+1}$ almost everywhere on $R^{n+1}$. Solving this system for $a \neq 0$ we get:

29) $F_{w}(p ; \zeta)=a^{-1} b_{1}-\left[\left(a^{2}-b^{2}+c^{2}\right)^{2}+4 b^{2} c^{2}\right]^{-1} a^{-1}$

$$
\left[\left(a^{2}-b^{2}+c^{2}\right)\left(\left(c^{2}-b^{2}\right) b_{1}+a b b_{2}-2 b c b_{3}+a c b_{4}\right)-2 b c\left(2 b c b_{1}-a c b_{2}+\left(c^{2}-b^{2}\right) b_{3}+a b b_{4}\right)\right] \text {. }
$$

Finally Formula (23) provides the expression for $f$ on the corresponding domain $W$ for suitable known function $g$ for which integrals converge. If $\gamma_{j}>0$ for each $j$, then $a>0$ for each $p_{3}^{2}+\cdots+p_{w}^{2}>0$.
For $(21,24)$ on a bounded domain with given boundary conditions equations will be of an analogous type with a term on the right $F^{n}(g(t), u ; p ; \zeta)$ minus boundary terms appearing in (6) in these particular cases.

For a partial differential equation

30) $a\left(t_{n+1}\right) A f\left(t_{1}, \cdots, t_{n+1}\right)+\partial f\left(t_{1}, \cdots, t_{n+1}\right) / \partial t_{n+1}=g\left(t_{1}, \cdots, t_{n+1}\right)$

with octonion valued functions $f, g$, where $A$ is a partial differential operator by variables $t_{1}, \cdots, t_{n}$ of the type given by $(2,2.1)$ with coefficients independent of $t_{1}, \cdots, t_{n}$, it may be simpler the following procedure. If a domain $V$ is not the entire Euclidean space $R^{n+1}$ we impose boundary conditions as above in (5.1). Make the noncommutative transform $F^{n ; t_{1}, \cdots, t_{n}}$ of both sides of Equation (30), so it takes the form:

31) $a\left(t_{n+1}\right) F^{n ; t_{1}, \cdots, t_{n}}\left(A f\left(t_{1}, \cdots, t_{n+1}\right), u ; p ; \zeta\right)+\partial F^{n ; t_{1}, \cdots, t_{n}}\left(f\left(t_{1}, \cdots, t_{n+1}\right), u ; p ; \zeta\right) / \partial t_{n+1}$

$$
=F^{n ; t_{1}, \cdots, t_{n}}\left(g\left(t_{1}, \cdots, t_{n+1}\right), u ; p ; \zeta\right) .
$$

In the particular case, when

$$
a\left(t_{n+1}\right) \sum_{|j| \leq \alpha} a_{j}\left(t_{n+1}\right) \sum_{0 \leq k_{1} \leq j_{1}}\left(\begin{array}{l}
j_{1} \\
k_{1}
\end{array}\right) S_{\left(k_{1}, j_{2}, \cdots, j_{k}\right)} e^{-u(p, t ; \zeta)}=e^{-u(p, t ; \zeta)}
$$

for each $t_{n+1}, p, t$ and $\zeta$, with the help of $(6,8)$ one can deduce an expression of

$$
F^{n}\left(p ; \zeta ; t_{n+1}\right)=\exp \left\{-\int_{\tau_{0}}^{t_{n+1}} b\left(p_{0}, \cdots, p_{n} ; \xi\right) \mathrm{d} \xi\right\}\left\{C_{0}+\left[\int_{\tau_{0}}^{t_{n+1}} Q\left(p_{0}, \cdots, p_{n} ; \tau\right) \exp \left\{\int_{\tau_{0}}^{t_{n+1}} b\left(p_{0}, \cdots, p_{n} ; \xi\right) \mathrm{d} \xi\right\} \mathrm{d} \tau\right]\right\}
$$

through

$$
G^{n}\left(p ; \zeta ; t_{n+1}\right):=F^{n ; t_{1}, \cdots, t_{n}}\left(g\left(t_{1}, \cdots, t_{n+1}\right), u ; p ; \zeta\right)
$$

and boundary terms in the following form:

$$
b\left(p_{0}, \cdots, p_{n} ; t_{n+1}\right) F^{n}\left(p ; \zeta ; t_{n+1}\right)+\partial F^{n}\left(p ; \zeta ; t_{n+1}\right) / \partial t_{n+1}=Q\left(p_{0}, \cdots, p_{n} ; t_{n+1}\right),
$$


where $b\left(p_{0}, \cdots, p_{n} ; t_{n+1}\right)$ is a real mapping and $Q\left(p_{0}, \cdots, p_{n} ; t_{n+1}\right)$ is an octonion valued function. The latter differential equation by $t_{n+1}$ has a solution ana- logously to the real case, since $t_{n+1}$ is the real va- riable, while $R$ is the center of the Cayley-Dickson algebra $A_{r}$. Thus we infer:

33) $F^{n}\left(p ; \zeta ; t_{n+1}\right)=\exp \left\{-\int_{\tau_{0}}^{t_{n+1}} b\left(p_{0}, \cdots, p_{n} ; \xi\right) \mathrm{d} \xi\right\}\left\{C_{0}+\left[\int_{\tau_{0}}^{t_{n+1}} Q\left(p_{0}, \cdots, p_{n} ; \tau\right) \exp \left\{\int_{\tau_{0}}^{t_{n+1}} b\left(p_{0}, \cdots, p_{n} ; \xi\right) \mathrm{d} \xi\right\} \mathrm{d} \tau\right]\right\}$

since the octonion algebra is alternative and each equation $b x=c$ with non-zero $b$ has the unique solution $x=b^{-1} c$, where $C_{0}$ is an octonion constant which can be specified by an initial condition. More general partial differential equations as (30), but with $\partial^{l} f / \partial t_{n+1}^{l}, l \geq 2$, instead of $\partial f / \partial t_{n+1}$ can be considered. Making the inverse transform $\left(F^{n ; t_{1}, \cdots, t_{n}}\right)^{-1}$ of the right side of (33) one gets the particular solution $f$.

\subsubsection{Integral Kernel}

We rewrite Equation 28(6) in the form:

34) $A_{S} F^{n}\left(f \chi_{Q^{n}}, u ; p ; \zeta\right)=F^{n}\left(g \chi_{Q^{n}}, u ; p ; \zeta\right)$

$$
\begin{gathered}
\sum_{|j| \leq \alpha} a_{j} \sum_{1 \leq|(j)|, 0 \leq m_{k}, 0 \leq q_{k}, h_{k}=\operatorname{sign}\left(l_{k} j_{k}\right), m_{k}+q_{k}+h_{k}=j_{k} ; q_{k}=0} \text { for } l_{k} j_{k}=0 ; \forall k=1, \cdots, n ;(l) \in\{0,1,2\}^{n} \\
(-1)^{|(j)| \mid} S^{m} F^{n-|h(j)|}\left(\left(\partial^{|q|} f(t)^{(j)} / \partial t_{1}^{q_{1}} \cdots \partial t_{n}^{q_{n}}\right) \chi_{\partial Q_{(j j)}^{n}}\left(t^{(l j)}\right), u ; p ; \zeta\right),
\end{gathered}
$$

where

$$
\text { 34.1) } S_{k}(p):=S_{k}:=R_{e_{k}}(p)
$$

in the $A_{r}$ spherical or $A_{r}$ Cartesian coordinates respectively (see also Formulas 25(1.1,1.2)), for each $k=1, \cdots, n$,

34.2) $S^{m}(p):=S^{m}:=S_{1}^{m_{1}} \cdots S_{n}^{m_{n}}$,

35) $A_{S}:=\sum_{|j| \leq \alpha} a_{j} S^{j}(p)$.

Then we have the integral formula:

$$
\text { 36) } \begin{aligned}
& A_{S} F^{n}\left(f \chi_{Q^{n}}, u ; p ; \zeta\right) \\
= & \int_{Q^{n}} f(t)\left[A_{S} \exp (-u(p, t ; \zeta))\right] \mathrm{d} t
\end{aligned}
$$

in accordance with 1(7) and 2(4). Due to $\S 28.3$ the operator $A_{S}$ has the inverse operator for $\lambda_{n+1}$ almost all $\left(p_{0}, \cdots, p_{n}\right)$ in $R^{n+1}$. Practically, its calculation may be cumbersome, but finding for an integral inversion formula its kernel is sufficient. In view of the inversion Theorem 6 or Corollary 6.1 and $\S \S 19$ and 20 we have

$$
\text { 37) } \begin{aligned}
& (2 \pi)^{-n} \int_{R^{n}} \exp (-u(a+p, t ; \zeta)) \\
& \exp (u(a+p, \tau ; \zeta)) \mathrm{d} p_{1} \cdots \mathrm{d} p_{n}=\delta(t ; \tau),
\end{aligned}
$$

where

$$
\text { 38) }[\delta, f)(\tau)=\int_{R^{n}} f(t) \delta(t ; \tau) \mathrm{d} t_{1} \cdots \mathrm{d} t_{n}=f(\tau)
$$

at each point $\tau \in R^{n}$, where the original $f(\tau)$ satisfies Hölder's condition. That is, the functional $\delta(t ; \tau)$ is $A_{r}$ linear. Thus the inversion of Equation (36) is:

39) $\int_{R^{n}}\left(\int_{R^{n}} f(t) \chi_{Q^{n}}(t)\left\{\left[A_{S} \exp (-u(p+a, t ; \zeta))\right] \xi(p+a, t, \tau ; \zeta)\right\} \mathrm{d} t\right) \mathrm{d} p_{1} \cdots \mathrm{d} p_{n}=f(\tau)$,

so that

40) $\left[A_{S} \exp (-u(p+a, t ; \zeta))\right] \xi(p+a, t, \tau ; \zeta)=(2 \pi)^{-n} \exp (-u(p+a, t ; \zeta)) \exp (-u(p+a, \tau ; \zeta))$,

where the coefficients of $A_{S}$ commute with generators $i_{j}$ of the Cayley-Dickson algebra $A_{r}$ for each $j$. Consider at first the alternative case, i.e. over the CayleyDickson algebra $A_{r}$ with $r \leq 3$.

Let by our definition the adjoint operator $A_{S}^{*}$ be defined by the formula

$$
\text { 41) } A_{S}^{*} \eta(p, t ; \zeta)=\sum_{|j| \leq \alpha} a_{j}^{*} S^{j} \eta^{*}(p, t ; \zeta)
$$

for any function $\eta: A_{r} \times R^{n} \times A_{r} \rightarrow A_{r}$, where $]^{*} t \in R^{n}$, $p$ and $\zeta \in A_{r}, \quad S^{j} \eta^{*}(p, t ; \zeta):=\left[S^{j} \eta(p, t ; \zeta)\right]^{*}$. Any Cayley-Dickson number $z \in A_{v}$ can be written with the help of the iterated exponent (see $\S 3$ ) in $A_{v}$ spherical coordinates as

42) $z=|z| \exp (-u(0,0 ; \psi))$,

where $v \geq r, \psi \in A_{v}, u \in A_{v}, \operatorname{Re}(\psi)=0$. Certainly 
the phase shift operator is isometrical:

43) $\left|T_{1}^{k_{1}} \cdots T_{n}^{k_{n}} z\right|=|z|$

for any $k_{1}, \cdots, k_{n} \in R$, since $\mid \exp (-u(0,0 ; \operatorname{Im}(\psi)) \mid=1$ for each $\psi \in A_{v}$, while

$$
\begin{aligned}
& T_{1}^{k_{1}} \cdots T_{n}^{k_{n}} e^{-u(0,0 ; \operatorname{Im}(\psi))}= \\
& \exp \left\{-u\left(0,0 ; \operatorname{Im}(\psi)-\left(k_{1} i_{1}+\cdots+k_{n} i_{n}\right) \pi / 2\right)\right\}
\end{aligned}
$$

(see $\S 12$ ).

In the $A_{r}$ Cartesian coordinates each Cayley-Dickson number can be presented as:

42.1) $z=|z| \exp (\phi M)$, where $\phi \in R$ is a real parameter, $M$ is a purely imaginary Cayley-Dickson number (see also $\S 3$ in $[5,6]$ ). Therefore, we deduce that

44) $\left|A_{S} \exp (-u(p+a, t ; \zeta))\right|$

$$
\begin{aligned}
& =\exp \left(-\left(p_{0}+a\right) s_{1}-\zeta_{0}\right) \\
& \left|A_{S} \exp (-u(\operatorname{Im}(p), t ; \operatorname{Im}(\zeta)))\right|
\end{aligned}
$$

since $R$ is the center of the Cayley-Dickson algebra $A_{v}$ and $p_{0}, a, \zeta_{0}, s_{1} \in R, s_{1}=s_{1}(t)$, where particularly $A_{S} 1:=\left.A_{S} e^{-u(0,0 ; \zeta)}\right|_{\zeta=0}$ (see also Formulas 12(3.1-3.7).
Then expressing $\xi$ from (40) and using Formulas $(41,42,42.1,44)$ we infer, that

45) $\xi(p, t, \tau ; \zeta)$

$$
\begin{aligned}
= & (2 \pi)^{-n}\left[A_{S}^{*} \exp (-u(\operatorname{Im}(p), t ; \operatorname{Im}(\zeta)))\right] \\
& {[\exp (-u I(m(p), t ; \operatorname{Im}(\zeta)) \exp (u(p, \tau ; \zeta)))], } \\
& \left|A_{S} \exp (-u(\operatorname{Im}(p), t ; \operatorname{Im}(\zeta)))\right|^{-2}
\end{aligned}
$$

since $z^{-1}=z^{*} /|z|^{2}$ for each non-zero Cayley-Dickson number $z \in A_{v}, v \geq 1$, where $\operatorname{Im}(p)=p_{1} i_{1}+\cdots+p_{n} i_{n}$, $p=p_{0} i_{0}+\cdots+p_{n} i_{n}, \quad p_{0}=\operatorname{Re}(p)$.

Generally, for $r \geq 4$, Formula (45) gives the integral kernel $\xi(p, t, \tau ; \zeta)$ for any restriction of $\xi$ on the octonion subalgebra $\operatorname{alg}_{R}\left(N_{1}, N_{2}, N_{4}\right)$ embedded into $A_{r}$. In view of $\S 28.3 \xi$ is unique and is defined by (45) on each subalgebra $\operatorname{alg}_{R}\left(N_{1}, N_{2}, N_{4}\right)$, consequently, Formula (45) expresses $\xi$ by all variables $p, \xi \in A_{r}$ and $t, \tau \in R^{n}$. Applying Formulas $(39,45)$ and $28.2(\Delta)$ to Equation (34), when Condition $8(3)$ is satisfied, we deduce, that

$$
\begin{aligned}
& \text { 46) }\left(f \chi_{Q^{n}}\right)(\tau)=\int_{R^{n}}\left(\int_{R^{n}} g(t) \chi_{Q^{n}}(t)[\exp (-u(p+a, t ; \zeta)) \xi(p+a, t, \tau ; \zeta)] \mathrm{d} t\right) \mathrm{d} p_{1} \cdots \mathrm{d} p_{n} \\
& -\sum_{|j| \leq \alpha} a_{j} \sum_{1 \leq(l j) \mid, 0 \leq m_{k}, 0 \leq q_{k}, h_{k}=\operatorname{sign}\left(l_{k} j_{k}\right) ; m_{k}+q_{k}+h_{k}=j_{k} ; q_{k}=0 \text { for } l_{k} j_{k}=0, \forall k=1, \ldots, n ;(l) \in\{0,1,2\}^{n}}(-1)^{|(l j)|} \\
& \int_{R^{n}}\left(\int_{\partial Q_{(l j)}^{n}}\left[\partial^{|q|} f\left(t^{(l j)} / \partial t_{1}^{q_{1}} \cdots \partial t_{n}^{q_{n}}\right]\left[\left\{S^{m}(p) \exp \left(-u\left(p+a, t^{(l j)} ; \zeta\right)\right)\right\} \xi\left(p+a, t^{(l j)}, \tau ; \zeta\right)\right] \mathrm{d} t^{(l j)}\right) \mathrm{d} p_{1} \cdots \mathrm{d} p_{n},\right.
\end{aligned}
$$

where $\operatorname{dim}_{R} \partial Q_{(l j)}^{n}=n-|h(l j)|, t^{(l j)} \in \partial Q_{(j)}^{n}$ in accordance with $\S 28.1, S^{m}(p)$ is given by Formulas $(34.1,34.2)$ above.

For simplicity the zero phase parameter $\zeta=0$ in (46) can be taken. In the particular case $Q^{n}=R^{n}$ all terms with $\int_{\partial Q_{(l j)}^{n}}$ vanish.

Terms of the form

47)

$$
A_{S}=\left(\sum_{h=1}^{n} a_{h}\left[S_{h}(p)\right]^{2}\right)+\beta_{n} S_{n}(p)+\omega
$$

and

$$
\text { 48) } \begin{aligned}
( & \left.f \chi_{U}\right)(t)=\int_{R^{n}}\left(\int_{R^{n}} g(t) \chi_{U}(t)[\exp (-u(p+a, t ; \zeta)) \xi(p, t, \tau ; \zeta)] \mathrm{d} t\right) \mathrm{d} p_{1} \cdots \mathrm{d} p_{n} \\
- & \int_{R^{n}}\left(\int_{\partial U_{0}} f\left(t^{\prime}\right)\left[\left\{\left(\beta\left(t^{\prime}\right)+P\left(t^{\prime}, p\right)\right) \exp (-u(p+a, t ; \zeta))\right\} \xi\left(p, t^{\prime}, \tau ; \zeta\right)\right] \mathrm{d} t^{\prime}\right) \mathrm{d} p_{1} \cdots \mathrm{d} p_{n} \\
& -\int_{R^{n}}\left(\int_{\partial U_{0}} a\left(t^{\prime}\right)\left(\partial f\left(t^{\prime}\right) / \partial v\right)\left[\exp (-u(p+a, t ; \zeta)) \xi\left(p, t^{\prime}, \tau ; \zeta\right)\right] d t^{\prime}\right) \mathrm{d} p_{1} \cdots \mathrm{d} p_{n} .
\end{aligned}
$$

For a calculation of the appearing integrals the generalized Jordan lemma (see $\S \quad \S 23$ and 24 in [4]) and residues of functions at poles corresponding to zeros

$$
\begin{aligned}
& \int_{R^{n}}\left[\left\{S^{m}(p) \exp (-u(p+a, t ; \zeta))\right\} \xi(p+a, t, \tau ; \zeta)\right] \\
& \mathrm{d} p_{1} \cdots \mathrm{d} p_{n}
\end{aligned}
$$

in Formula (46) can be interpreted as left $A_{r}$ linear functionals due to Fubini's theorem and $\S \quad \S \quad 19$ and 20, where $S^{0}=I$.

For the second order operator from (14) one gets:
$\left|A_{S} \exp (-u(\operatorname{Im}(p), t ; \operatorname{Im}(\zeta)))\right|=0$ by variables $p_{1}, \cdots, p_{n}$ can be used.

Take $g(t)=\delta(y ; t)$, where $y \in R^{n}$ is a parameter, then 
49) $\int_{R^{n}}\left(\int_{R^{n}} \delta(y ; t)[\exp (-u(p+a, t ; \zeta)) \xi(p+a, t, \tau ; \zeta)] \mathrm{d} t\right) \mathrm{d} p_{1} \cdots \mathrm{d} p_{n}$ $=\int_{R^{n}}[\exp (-u(p+a, y ; \zeta)) \xi(p+a, y, \tau ; \zeta)] \mathrm{d} p_{1} \cdots \mathrm{d} p_{n}=: E(y ; \tau)$

is the fundamental solution in the class of generalized functions, where

50) $A_{t} E(y ; t)=\delta(y ; t)$,

51) $\int_{R^{n}} \delta(y ; t) f(t) \mathrm{d} t=f(y)$

for each continuous function $f(t)$ from the space $L^{2}\left(R^{n}, A_{r}\right) ; A_{t}$ is the partial differential operator as above acting by the variables $t=\left(t_{1}, \cdots, t_{n}\right)$ (see also $\S$ $\S 19,20$ and 33-35).

\subsection{The Decomposition Theorem of Partial Differential Operators over the Cayley-Dickson Algebras}

We consider a partial differential operator of order $u$ :

1) $A f(x)=\sum_{|\alpha| \leq u} a_{\alpha}(x) \partial^{\alpha} f(x)$,

where $\partial^{\alpha} f=\partial^{|\alpha|} f(x) / \partial x_{0}^{\alpha_{0}} \cdots \partial x_{n}^{\alpha_{n}}, \quad x=x_{0} i_{0}+\cdots x_{n} i_{n}$, $x_{j} \in R$ for each $j, 1 \leq n=2^{r}-1, \alpha=\left(\alpha_{0}, \cdots, \alpha_{n}\right)$, $|\alpha|=\alpha_{0}+\cdots+\alpha_{n}, \quad 0 \leq \alpha_{j} \in Z$. By the definition this means that the principal symbol

2) $A_{0}:=\sum_{|\alpha|=u} a_{\alpha}(x) \partial^{\alpha}$

has $\alpha$ so that $|\alpha|=u$ and $a_{\alpha}(x) \in A_{r}$ is not identically zero on a domain $U$ in $A_{r}$. As usually

$C^{k}\left(U, A_{r}\right)$ denotes the space of $k$ times continuously differentiable functions by all real variables $x_{0}, \cdots, x_{n}$ on $U$ with values in $A_{r}$, while the $x$-differentiability corresponds to the super-differentiability by the CayleyDickson variable $x$.

Speaking about locally constant or locally differentiable coefficients we shall undermine that a domain $U$ is the union of subdomains $U^{j}$ satisfying conditions 28(D1,i-vii) and $U^{j} \cap U^{k}=\partial U^{j} \cap \partial U^{k}$ for each $j \neq k$. All coefficients $a_{\alpha}$ are either constant or differentiable of the same class on each $\operatorname{Int}\left(U^{j}\right)$ with the continuous extensions on $U^{j}$. More generally it is up to a $C^{u}$ or $x$-differentiable diffeomorphism of $U$ respectively.

If an operator $A$ is of the odd order $u=2 s-1$, then an operator $E$ of the even order $u+1=2 s$ by variables $(t, x)$ exists so that

3) $\left.\operatorname{Eg}(t, x)\right|_{t=0}=\operatorname{Ag}(0, x)$

for any $g \in C^{u+1}\left([c, d] \times U, A_{r}\right)$, where $t \in[c, d] \subset R$, $c \leq 0<d$, for example, $\operatorname{Eg}(t, x)=\partial(\operatorname{tag}(t, x))\rangle \partial t$.

Therefore, it remains the case of the operator $A$ of the even order $u=2 s$. Take

$z=z_{0} i_{0}+\cdots+z_{2^{v}-1} i_{2^{v}-1} \in A_{v}, \quad z_{j} \in R$. Operators depending on a less set $z_{l_{1}}, \cdots, z_{l_{n}}$ of variables can be consi- dered as restrictions of operators by all variables on spaces of functions constant by variables $z_{s}$ with $s \notin\left\{l_{1}, \cdots, l_{n}\right\}$.

Theorem. Let $A=A_{u}$ be a partial differential operator of an even order $u=2 s$ with locally constant or variable $C^{s}$ or $x$-differentiable on $U$ coefficients $a_{\alpha}(x) \in A_{r}$ such that it has the form

4) $A f=c_{u, 1}\left(B_{u, 1} f\right)+\cdots+c_{u, k}\left(B_{u, k} f\right)$,

where each

5) $B_{u, p}=B_{u, p, 0}+Q_{u-1, p}$ is a partial differential operator by variables $x_{m_{u, 1}+\cdots+m_{u, p-1}+1}, \cdots, x_{m_{u, 1}+\cdots+m_{u, p}}$ and of the order $u$, $m_{u, 0}=0, c_{u, k}(x) \in A_{r}$ for each $k$, its principal part

6) $B_{u, p, 0}=\sum_{|\alpha|=s} a_{p, 2 \alpha}(x) \partial^{2 \alpha}$

is elliptic with real coefficients $a_{p, 2 \alpha}(x) \geq 0$, either $0 \leq r \leq 3$ and $f \in C^{u}\left(U, A_{r}\right)$, or $r \geq 4$ and

$f \in C^{u}(U, R)$. Then three partial differential operators $\Upsilon^{s}$ and $\Upsilon_{1}^{s}$ and $Q$ of orders $s$ and $p$ with $p \leq u-1$ with locally constant or variable $C^{s}$ or $x$ differentiable correspondingly on $U$ coefficients with values in $A_{v}$ exist, $r \leq v$, such that

7) $A f=\Upsilon^{s}\left(\Upsilon_{1}^{s} f\right)+Q f$.

\subsection{Corollary 1}

Let suppositions of Theorem 29 be satisfied. Then a change of variables locally constant or variable $C^{1}$ or $x$-differentiable on $U$ correspondingly exists so that the principal part $A_{2,0}$ of $A_{2}$ becomes with constant coefficients, when $a_{p, 2 \alpha}>0$ for each $p, \alpha$ and $x$.

\subsection{Corollary 2}

If two operators $E=A_{2 s}$ and $A=A_{2 s-1}$ are related by Equation 29(3), and $A_{2 s}$ is presented in accordance with Formulas 29(4,5), then three operators $\Upsilon^{s}, \Upsilon^{s-1}$ and $Q$ of orders $s, s-1$ and $2 s-2$ exist so that

1) $A_{2 s-1}=\Upsilon^{s} \Upsilon^{s-1}+Q$.

\subsection{Products of Operators}

We consider operators of the form:

1) $\left(\Upsilon^{k}+\beta I_{r}\right) f(z):=$

$$
\left\{\sum_{0<|\alpha| \leqslant k} \partial^{\alpha} f(z) \eta_{\alpha}(z)\right\}+f(z) \beta(z)
$$

with $\eta_{\alpha}(z) \in A_{v}, \quad \alpha=\left(\alpha_{0}, \cdots, \alpha_{2^{r}-1}\right), \quad 0 \leq \alpha_{k} \in N$ for each $k,|\alpha|=\alpha_{0}+\cdots+\alpha_{2^{r}-1}, \beta I_{r} f(z):=f(z) \beta$, 
$\partial^{\alpha} f(z):=\partial^{|\alpha|} f(z) / \partial z_{0}^{\alpha} \cdots \partial z_{2^{r}-1}^{\alpha^{r}-1}, 2 \leq r \leq v<\infty$, $\beta(z) \in A_{v}, \quad z_{0}, \cdots, z_{2^{r}-1} \in R, \quad z=z_{0} i_{0}+\cdots+z_{2^{r}-1} i_{2^{r}-1}$.

Proposition. The operator $\left(\Upsilon^{k}+\beta\right)^{*}\left(\Upsilon^{k}+\beta\right)$ is elliptic on the space $C^{2 k}\left(R^{2^{r}}, A_{v}\right)$.

\subsection{Fundamental Solutions}

Let either $Y$ be a real $Y=A_{v}$ or complexified $Y=\left(A_{v}\right)_{C}$ or quaternionified $Y=\left(A_{v}\right)_{H}$ Cayley-Dickson algebra (see $\S 28$ ). Consider the space $B\left(R^{n}, Y\right)$ (see $\S 19$ ) supplied with a topology in it is given by the countable family of semi-norms

1) $p_{\alpha, k}(f):=\sup _{x \in R^{n}}\left|(1+|x|)^{k} \partial^{\alpha} f(x)\right|$,

where $k=0,1,2, \cdots ; \alpha=\left(\alpha_{1}, \cdots, \alpha_{n}\right), \quad 0 \leq \alpha_{j} \in Z$. On this space we take the space $B^{\prime}\left(R^{n}, Y\right)$ of all $Y$ valued continuous generalized functions (functionals) of the form

2) $\begin{aligned} f & =f_{0} i_{0}+\cdots+f_{2^{v}-1} i_{2^{v}-1} \text { and } \\ g & =g i_{0}+\cdots+g\end{aligned}$

$g=g_{0} i_{0}+\cdots+g_{2^{v}-12^{v}-1}$,

where $f_{j}$ and $g_{j} \in B^{\prime}\left(R^{n}, Y\right)$, with restrictions on $B\left(R^{n}, R\right)$ being real or $C_{i}$ or $H_{J, K, L}$-valued generalized functions $f_{0}, \cdots, f_{2^{v}-1}, g_{0}, \cdots, g_{2^{v}-1}$ respectively. Let $\phi=\phi_{0} i_{0}+\cdots+\phi_{2^{v}-1} i_{2^{v}-1}$ with $\phi_{0}, \cdots, \phi_{2^{v}-1} \in B\left(R^{n}, R\right)$, then

3) $[f, \phi)=\sum_{k, j=0}^{2^{v}-1}\left[f_{j}, \phi_{k}\right) i_{k} i_{j}$.

We define their convolution as

4) $\left[f^{*} g, \phi\right)=\sum_{j, k=0}^{2^{v}-1}\left(\left[f^{*} g, \phi\right) i_{j}\right) i_{k}$

for each $\phi \in B\left(R^{n}, Y\right)$. As usually

5) $\left(f^{*} g\right)(x)=f(x-y) * g(y)=f(y) * g(x-y)$

for all $x, y \in R^{n}$ due to (4), since the latter Equality (5) is satisfied for each pair $f_{j}$ and $g_{k}$. Thus a solution of the equation

6) $\left(\Upsilon^{s}+\beta\right) f=g$ in $B\left(R^{n}, Y\right)$ or in the space $B^{\prime}\left(R^{n}, Y\right)$ is:

7) $f \stackrel{l}{=} E_{\Upsilon^{s}+\beta} * g$, where $E_{\Upsilon^{s}+\beta}$ denotes a fundamental solution of the equation

8) $\left(\Upsilon^{s}+\beta\right) E_{\Upsilon+\beta}=\delta,(\delta, \phi)=\phi(0)$.

The fundamental solution of the equation

9) $A_{0} V=\delta$ with $A_{0}=\left(\Upsilon^{s}+\beta\right)\left(\Upsilon_{1}^{s_{1}}+\beta_{1}\right)$

using Equalities $32(2-4)$ can be written as the convolution

10) $V=: V_{A_{0}}=E_{\Upsilon^{s}+\beta} * E_{\Upsilon_{1}^{s_{1}}+\beta_{1}}$.

More generally we can consider the equation

11) $A f=g$ with $A=A_{0}+\left(\Upsilon_{2}+\beta_{2}\right)$, where $A_{0}=(\Upsilon+\beta)\left(\Upsilon_{1}+\beta_{1}\right), \Upsilon, \Upsilon_{1}, \Upsilon_{2}$ are operators of orders $s, s_{1}$ and $s_{2}$ respectively given by 32(1) with $z$-differentiable coefficients. For $\Upsilon_{2}+\beta_{2}=0$ this equation was solved above. Suppose now, that the operator $\Upsilon_{2}+\beta_{2}$ is non-zero.

To solve Equation (11) on a domain $U$ one can write it as the system:

12) $\left(\Upsilon_{1}+\beta_{1}\right) f=g_{1},(\Upsilon+\beta) g_{1}=g-\left(\Upsilon_{2}+\beta_{2}\right) f$.

Find at first a fundamental solution $V_{A}$ of Equation (11) for $g=\delta$. We have:

13) $f=E_{\Upsilon_{1}+\beta_{1}} * g_{1}=E_{\Upsilon_{2}+\beta_{2}} *\left(g-(\Upsilon+\beta) g_{1}\right)$, consequently,

13. 1) $E_{\Upsilon_{1}+\beta_{1}} * g_{1}+E_{\Upsilon_{2}+\beta_{2}} *\left((\Upsilon+\beta) g_{1}\right)=E_{\Upsilon_{2}+\beta_{2}} * g$. In accordance with (3-5) and 32(1) the identity is satisfied: $\left[E_{\Upsilon_{2}+\beta_{2}} *\left((\Upsilon+\beta) g_{1}\right), \phi_{0}\right)=\left[(\Upsilon+\beta)\left(E_{\Upsilon_{2}+\beta_{2}} * g_{1}\right), \phi_{0}\right)$.

Thus (13) is equivalent to

14) $E_{\Upsilon_{1}+\beta_{1}} * g_{1}+(\Upsilon+\beta)\left(E_{\Upsilon_{2}+\beta_{2}} * g_{1}\right)=E_{\Upsilon_{2}+\beta_{2}}$

for $g=\delta$, since $E_{\Upsilon_{2}+\beta_{2}} * \delta=E_{\Upsilon_{2}+\beta_{2}}$.

We consider the Fourier transform $F$ by real variables with the generator $i$ commuting with $i_{j}$ for each $j=0, \cdots, 2^{v}-1$ such that

$$
(F 1)(F g)(y)=\int_{R^{n}} e^{-i(y, x)} g(x) \mathrm{d} x_{1} \cdots \mathrm{d} x_{n}
$$
for any $g \in L^{1}\left(R^{n}, A_{v}\right)$, i.e. $\int_{R^{n}}|g(x)| \mathrm{d} x_{1} \cdots \mathrm{d} x_{n}<\infty$, where $g: R^{n} \rightarrow Y$ is an integrable function, $(y, x)=x_{1} y_{1}+\cdots+x_{n} y_{n}, \quad x=\left(x_{1}, \cdots, x_{n}\right) \in R^{n}, \quad x_{j} \in R$ for every $j$. The inverse Fourier transform is:

$$
(F 2)\left(F^{-1} g\right)(y)=(2 \pi)^{-n} \int_{R^{n}} e^{i(y, x)} g(x) \mathrm{d} x_{1} \cdots \mathrm{d} x_{n} .
$$

For a generalized function $f$ from the space $B^{\prime}\left(R^{n}, Y\right)$ its Fourier transform is defined by the formula

$$
(F 3)(F f, \phi)=(f, F \phi),\left(F^{-1} f, \phi\right)=\left(f, F^{-1} \phi\right) .
$$

In view of (2-5) the Fourier transform of (14) gives:

$$
\text { 15) } \begin{aligned}
{[} & \left.F\left(E_{\Upsilon_{1}+\beta_{1}}\right)\right]\left[F\left(g_{1}\right)\right] \\
& +\sum_{j=0}^{2^{v}-1}\left[F\left((\Upsilon+\beta)_{j} E_{\Upsilon_{2}+\beta_{2}}\right)\right] \\
& {\left[F\left(g_{1}\right)\right] i_{j}=F\left(E_{\Upsilon_{2}+\beta_{2}}\right) }
\end{aligned}
$$

for $g=\delta$. With generators $i_{0}, \cdots, i_{2^{v}-1}, i_{0} i, \cdots, i_{2^{v}-1} i$ the latter equation gives the linear system of $2^{v+1}$ equations over the real field, or $2^{v+2}$ equations when $Y=\left(A_{v}\right)_{H}$. From it $F\left(g_{1}\right)$ and using the inverse transform $F^{-1}$ a generalized function $g_{1}$ can be found, since

$$
\begin{aligned}
& F(g)=F\left(g_{0}\right) i_{0}+\cdots+F\left(g_{2^{v}-1}\right) i_{2^{v}-1} \text { and } \\
& F^{-1}(g)=F^{-1}\left(g_{0}\right) i_{0}+\cdots+F^{-1}\left(g_{2^{v}-1}\right) i_{2^{v}-1} \text { (see also the }
\end{aligned}
$$

Fourier transform of real and complex generalized func- 
tions in [1,21]). Then

16) $V_{A}=E_{\Upsilon_{1}+\beta_{1}} * g_{1}$ and $f=V_{A} * g$ gives the solution of (11), where $g_{1}$ was calculated from (15).

Let $\pi_{r}^{v}:\left(A_{v}\right)_{H} \rightarrow\left(A_{r}\right)_{H}$ be the $R$-linear projection operator defined as the sum of projection operators $\pi_{0}+\cdots+\pi_{2^{r}-1}$, where $\pi_{j}:\left(A_{v}\right)_{H} \rightarrow H i_{j}$,

17) $\pi_{j}(h)=h_{j} i_{j}, \quad h=\sum_{j=0}^{2^{v}-1} h_{j} i_{j}, \quad h_{j} \in H_{J, K, L}$, that gives the corresponding restrictions when $h_{j} \in C_{i}$ or $h_{j} \in R$ for $j=0, \cdots, 2^{r}-1$. Indeed, Formulas $2(5,6)$ have the natural extension on $\left(A_{v}\right)_{H}$, since the generators $J$ $K$ and $L$ commute with $i_{j}$ for each $j$.

Finally, the restriction from the domain in $A_{v}$ onto the initial domain of real variables in the real shadow and the extraction of $\pi_{r}^{v} \circ f \in A_{r}$ with the help of Formulas 2(5,6) gives the reduction of a solution from $A_{v}$ to $A_{r}$.

Theorems 29, Proposition 32 and Corollaries 30, 31 together with formulas of this section provide the algorithm for subsequent resolution of partial differential equations for $s, s-1, \cdots, 2$, because principal parts of operators $A_{2}$ on the final step are with constant coefficients. A residue term $Q$ of the first order can be integrated along a path using a non-commutative line integration over the Cayley-Dickson algebra $[5,6]$.

\subsection{Multiparameter Transforms of Generalized Functions}

If $\phi \in B\left(R^{n}, Y\right)$ and $g \in B^{\prime}\left(R^{n}, Y\right)$, (see $\S \quad \S 19$ and 33) we put

$$
\text { 1) } \begin{aligned}
& \sum_{j=0}^{2^{v}-1}\left[F^{n}\left(g_{j} ; u ; p ; \zeta\right), \phi\right) i_{j} \\
& =\sum_{j=0}^{2^{v}-1}\left[g_{j}, F^{n}(\phi ; u ; p ; \zeta)\right) i_{j}
\end{aligned}
$$

or shortly

2) $\sum_{j=0}^{2^{v}-1}\left[g_{j} e^{-u(p ; ; ; \zeta)}, \phi\right) i_{j}=\sum_{j=0}^{2^{v}-1}\left[g_{j}, \phi e^{-u(p ; t ; 5)}\right) i_{j}$.

If the support $\operatorname{supp}(g)$ of $g$ is contained in a domain $U$, then it is sufficient to take a base function $\phi$ with the restriction $\left.\phi\right|_{U} \in B(U, Y)$ and any $\left.\operatorname{ss} \phi\right|_{R^{n} \backslash U} \in C^{\infty}$.

\subsection{Examples}

Let

$$
\text { 1) } A f(t)=\sum_{j=1}^{n}\left(\partial^{2} f(t) / \partial t_{j}^{2}\right) c_{j}
$$

be the operator with constant coefficients $c_{j} \in A_{r}$, $\left|c_{j}\right|=1$, by the variables $t_{1}, \cdots, t_{n}, n \geq 2$. We suppose that $c_{j}$ are such that the minimal subalgebra $\operatorname{alg}_{R}\left(c_{j}, c_{k}\right)$ containing $c_{j}$ and $c_{k}$ is alternative for each $j$ and $k$ and $\left|\left(\cdots\left(c_{1}^{1 / 2} c_{2}^{1 / 2}\right) \cdots\right) c_{n}^{1 / 2}\right|=1$. Since
2) $\partial f(t) / \partial t_{j}=\sum_{k=1}^{n}\left(\partial f(t(s)) / \partial s_{k}\right)\left(\partial s_{k} / \partial t_{j}\right)$

$$
=\sum_{k=1}^{j} \partial(t(s)) / \partial s_{k},
$$

the operator $A$ takes the form

$$
\text { 3) } A f=\sum_{j=1}^{n}\left(\sum_{1 \leq k, b \leq j}\left(\partial^{2} f(t(s)) / \partial s_{k} \partial s_{b}\right)\right) c_{j} \text {, }
$$

where $s_{j}=t_{j}+\cdots+t_{n}$ for each $j$. Therefore, by Theorem 12 and Formulas 25(SO) and 28(6) we get:

$$
\text { 4) } F^{n}(A f ; u ; p ; \zeta)=\sum_{j=1}^{n}\left\{\left[R_{e_{j}}(p)\right]^{2} F_{u}^{n}(p ; \zeta)\right\} c_{j}
$$

for $u(p, t ; \zeta)$ either in $A_{r}$ spherical or $A_{r}$ Cartesian coordinates with the corresponding operators $R_{e_{j}}(p)$ (see also Formulas 25(1.1,1.2)).

On the other hand,

$$
\text { 5) } F^{n}(\delta ; u ; p ; \zeta)=e^{-u(p, 0 ; \zeta)}=e^{-u(0,0 ; \zeta)}
$$

in accordance with Formula 20(2). The delta function $\delta(t)$ is invariant relative to any invertible linear operator $C: R^{n} \rightarrow R^{n}$ with the determinant $|\operatorname{det}(C)|=1$, since

$$
\begin{aligned}
\int_{R^{n}} \delta(C x) \phi(x) \mathrm{d} x & =\int_{R^{n}} \delta(y) \phi\left(C^{-1} y\right)|\operatorname{det}(C)| \mathrm{d} y \\
& =\phi\left(C^{-1} 0\right)=\phi(0) .
\end{aligned}
$$

Thus

$$
F^{n}(C(A f) ; u ; p ; \zeta)=F^{n}(A f ; u ; p ; \zeta)
$$

for any Fundamental solution $f$, where $C g(t):=g(C t), A f=\delta$. If $C: R^{n} \rightarrow R^{n}$ is an invertible linear operator and $\xi=C t, q=C p$, $\zeta^{\prime}=C \zeta$, then $t=C^{-1} \xi, p=C^{-1} q$ and $\zeta=C^{-1} \zeta^{\prime}$. In the multiparameter noncommutative transform $F^{n}$ there are the corresponding variables $\left(t_{j}, p_{j}, \zeta_{j}\right)$. This is accomplished in particular for the operator

$C\left(t_{1}, \cdots, t_{n}\right)=\left(s_{1}, \cdots, s_{n}\right)$. The operator $C^{-1}$ transforms the right side of Formula (4), when it is written in the $A_{r}$ spherical coordinates, into

$\sum_{j=1}^{n}\left\{\left(p_{0}+q_{j} S_{e_{j}}\right)^{2} F_{u}^{n}(q ; \zeta)\right\} c_{j}$. The Cayley-Dickson number $q=q_{0}+q_{1} i_{1}+\cdots+q_{n} i_{n}$ can be written as $q=q_{0}+q_{M} M$, where $|M|=1, M$ is a purely imaginary Cayley-Dickson number, $q_{M} \in R$, $q_{M} M=q_{1} i_{1}+\cdots+q_{n} i_{n}$, since $q_{0}=\operatorname{Re}(q)$. After a suitable automorphism $\theta: A_{r} \rightarrow A_{r}$ we can take $\theta(q)=q_{0}+q_{M} i_{1}$, so that $\theta(x)=x$ for any real number. The functions $\left[\sum_{j=1}^{n} q_{j}^{2} S_{e_{j}}^{2} c_{j}\right]$ and $\left[\sum_{j=1}^{n} p_{j}^{2} S_{e_{j}}^{2} c_{j}\right]$ are even by each variable $q_{j}$ and $p_{j}$ respectively. Therefore, we deduce in accordance with $(5)$ and $2(3,4)$ and Corollary 6.1 with parameters $p_{0}=0$ and $\zeta=0$ and $c_{j} \in\{-1,1\}$ for each $J$ that 
6) $\left(F^{n}\right)^{-1}$

$$
\begin{aligned}
& \left(1 /\left[\sum_{j=1}^{n}\left\{\sum_{1 \leq k, b \leq j} p_{k} S_{e_{k}} p_{b} S_{e_{b}}\right\} c_{j}\right] ; u ; y ; \zeta\right) \\
& =-\left[g, e^{N([y],[q])}\right)
\end{aligned}
$$

in the $A_{r}$ spherical coordinates, where

$$
\begin{aligned}
& g=1 / {\left[\sum_{j=1}^{n} q_{j}^{2} c_{j}\right], \text { or } } \\
& \text { 6.1) }\left(F^{n}\right)^{-1}\left(1 /\left[\sum_{j=1}^{n}\left\{p_{j}^{2} S_{e_{j}}^{2}\right\} c_{j}\right] ; u ; y ; \zeta\right) \\
&\left(F^{n}\right)^{-1}\left(1 /\left[\sum_{j=1}^{n}\left\{p_{j}^{2} S_{e_{j}}^{2}\right\} c_{j}\right] ; u ; y ; \zeta\right)
\end{aligned}
$$

in the $A_{r}$ Cartesian coordinates, where $g=1 /\left[\sum_{j=1}^{n} p_{j}^{2} c_{j}\right], \quad N=y /|y|$ for $y \neq 0, N=i_{1}$ for

7) $\left(F^{n}\right)^{-1}\left(1 /\left[\sum_{j=1}^{n}\left\{\sum_{1 \leq k, b \leq j} p_{k} S_{e_{k}} p_{b} S_{e_{b}}\right\} c_{j}\right] ; u ; y ; \zeta\right)$

$$
=(2 \pi)^{-n} \int_{R^{n}} \exp \left(i\left(q_{1} y_{1}+\cdots+q_{n} y_{n}\right)\right)\left(1 /\left[\sum_{j=1}^{k_{+}} q_{j}^{2}-\sum_{j=k_{+}+1}^{n} q_{j}^{2}\right]\right) \mathrm{d} q_{1} \cdots \mathrm{d} q_{n}
$$

in the $A_{r}$ spherical coordinates and

$$
\text { 7.1) }\left(F^{n}\right)^{-1}\left(1 /\left[\sum_{j=1}^{n} p_{j}^{2} S_{e_{j}}^{2} c_{j}\right] ; u ; y ; \zeta\right)=(2 \pi)^{-n} \int_{R^{n}} \exp \left(i\left(p_{1} y_{1}+\ldots+p_{n} y_{n}\right)\right)\left(1 /\left[\sum_{j=1}^{k_{+}} p_{j}^{2}-\sum_{j=k_{+}+1}^{n} p_{j}^{2}\right]\right) \mathrm{d} p_{1} \cdots \mathrm{d} p_{n}
$$

in the $A_{r}$ Cartesian coordinates, since for any even function its cosine Fourier transform coincides with the Fourier transform.

The inverse Fourier transform $\left(F^{-1} g\right)(x)=(2 \pi)^{-n}(F g)(-x)=: \Psi_{n}$ of the functions $g=1 /\left(\sum_{j=1}^{n} z_{j}^{2}\right)$ for $n \geq 3$ and $P\left(1 /\left(\sum_{j=1}^{2} z_{j}^{2}\right)\right)$ for $n=2$ in the class of the generalized functions is known

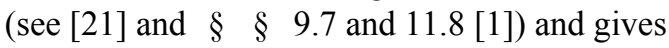

8) $\Psi_{n}\left(z_{1}, \cdots, z_{n}\right)=C_{n}\left(\sum_{j=1}^{n} z_{j}^{2}\right)^{1-n / 2}$

for $3 \leq n$, where $C_{n}=-1 /\left[(n-2) \sigma_{n}\right]$, $\sigma_{n}=4 \pi^{n / 2} / \Gamma((n / 2)-1)$ denotes the surface of the unit sphere in $R^{n}, \Gamma(x)$ denotes Euler's gamma-function, while

$$
\text { 9) } \Psi_{2}\left(z_{1}, z_{2}\right)=C_{2} \ln \left(\sum_{j=1}^{2} z_{j}^{2}\right)
$$

for $n=2$, where $C_{2}=1 /(4 \pi)$.

Thus the technique of $\S 2$ over the Cayley-Dickson algebra has permitted to get the solution of the Laplace operator.

For the function

$$
\text { 10) } P(x)=\sum_{j=1}^{k_{+}} x_{j}^{2}-\sum_{j=k_{+}+1}^{n} x_{j}^{2}
$$

with $1 \leq k_{+}<n$ the generalized functions $(P(x)+i 0)^{\lambda}$ and $(P(x)-i 0)^{\lambda}$ are defined for any $\lambda \in C=R \oplus i R$ (see Chapter 3 in [21]). The function $P^{\lambda}$ has the cone surface $P\left(z_{1}, \cdots, z_{n}\right)=0$ of zeros, so that for the correct definition of generalized functions corresponding to $P^{\lambda}$ the generalized functions

$$
\text { 11) } \begin{aligned}
(P(x)+c i 0)^{\lambda}=\lim _{0<c \varepsilon, \varepsilon \rightarrow 0}\left(P(x)^{2}+\varepsilon^{2}\right)^{\lambda / 2} \\
\exp (i \lambda \arg (P(x)+i c \varepsilon))
\end{aligned}
$$

with either $c=-1$ or $c=1$ were introduced. Therefore, the identity

12) $F\left(\Psi_{k_{+}, n-k_{+}}\right)(x)$

$$
=-\left(\sum_{j=1}^{k_{+}} x_{j}^{2}-\sum_{j=k_{+}+1}^{n} x_{j}^{2}\right)\left[F\left(\Psi_{k_{+}, n-k_{+}}\right)(x)\right]^{2}
$$

or

13) $F(\Psi)=-1 /(P(x)+c i 0)$

follows, where $c=-1$ or $c=1$.

The inverse Fourier transform in the class of the generalized functions is:

$$
\text { 14) } \begin{aligned}
F^{-1} & \left((P(x)+c i 0)^{\lambda}\right)\left(z_{1}, \cdots, z_{n}\right) \\
= & \exp \left(-\pi c\left(n-k_{+}\right) i / 2\right) 2^{2 \lambda+n} \pi^{n / 2} \Gamma(\lambda+n / 2) \\
& \left(Q\left(z_{1}, \cdots, z_{n}\right)-c i 0\right)^{-\lambda-n / 2)} /\left[\Gamma(-\lambda)|D|^{1 / 2}\right]
\end{aligned}
$$

for each $\lambda \in C$ and $n \geq 3$ (see $\S$ IV.2.6 [21]), where $D=\operatorname{det}\left(g_{j, k}\right)$ denotes a discriminant of the quadratic form $P(x)=\sum_{j, k=1}^{n} g_{j, k} x_{j} x_{k}$, while 
$Q(y)=\sum_{j, k=1}^{n} g^{j, k} x_{j} x_{k}$ is the dual quadratic form so that $\sum_{k=1}^{n} g^{j, k} g_{k, l}=\delta_{l}^{j}$ for all $j, l ; \delta_{l}^{j}=1$ for $j=l$ and $\delta_{l}^{j}=0$ for $j \neq l$. In the particular case of $n=2$ the inverse Fourier transform is given by the formula:

$$
\text { 15) } \begin{aligned}
F^{-1} & \left((P(x)+c i 0)^{-1}\right)\left(z_{1}, z_{2}\right) \\
= & -4^{-1}|D|^{-1 / 2} \exp \left(-\pi c\left(n-k_{+}\right) i / 2\right) \\
& \ln \left(Q\left(z_{1}, \cdots, z_{n}\right)-c i 0\right) .
\end{aligned}
$$

Making the inverse Fourier transform $F^{-1}$ of the function $-1 /(P(x)+i 0)$ in this particular case of $\lambda=-1$ we get two complex conjugated fundamental solutions

$$
\text { 16) } \begin{aligned}
& \Psi_{k_{+}, n-k_{+}}\left(z_{1}, \cdots, z_{n}\right) \\
= & -\exp \left(\pi c\left(n-k_{+}\right) i / 2\right) \Gamma((n / 2)-1) \\
& \left(Q\left(z_{1}, \cdots, z_{n}\right)+c i 0\right)^{1-(n / 2)} /\left(4 \pi^{n / 2}\right)
\end{aligned}
$$

for $3 \leq n$ and $1 \leq k_{+}<n$, while

17) $\Psi_{1,1}\left(z_{1}, z_{2}\right)=$

$$
4^{-1} \exp \left(\pi c\left(n-k_{+}\right) i / 2\right) \ln \left(Q\left(z_{1}, z_{2}\right)+c i 0\right)
$$

for $n=2$, where either $c=1$ or $c=-1$.

Generally for the operator $A$ given by Formula (1) we get $P(x)=P_{0}(x)+P_{i}(x)$, where

$$
P_{0}(x)=\sum_{j=1}^{n} x_{j}^{2} \operatorname{Re}\left(c_{j}\right) \text { and } P_{i}(x)=\sum_{j=1}^{n} x_{j}^{2} \operatorname{Im}\left(c_{j}\right) \text { are }
$$

the real and imaginary parts of $P, \operatorname{Im}(z)=z-\operatorname{Re}(z)$ for any Cayley-Dickson number $z$. Take $l=i_{2}$ and consider the form $P(x)+\varepsilon c l$ with $\varepsilon \neq 0$ and either $c=1$ or $c=-1$, then $P(x)+\varepsilon c l \neq 0$ for each $x \in R^{n}$. We put

$$
\text { 18) } \begin{aligned}
& (P(x)+c l 0)^{\lambda}=\lim _{0<c \varepsilon, \varepsilon \rightarrow 0}\left(P(x)^{2}+\varepsilon^{2}\right)^{\lambda / 2} \\
& \exp (i \lambda \operatorname{Arg}(P(x)+l c \varepsilon)) .
\end{aligned}
$$

Consider $\lambda \in R$, the generalized function

$$
\left(P(x)^{2}+\varepsilon^{2}\right)^{\lambda / 2} \exp (i \lambda \operatorname{Arg}(P(x)+l c \varepsilon))
$$

is non-degenerate and for it the Fourier transform is defined. The limit $\lim _{0<c \varepsilon, \varepsilon \rightarrow 0}$ gives by our definition the Fourier transform of $(P(x)+c l 0)^{\lambda}$. Since

$$
\text { 19) } c_{j}\left(\beta_{j}+\sum_{1 \leq k \leq n, k \neq j} c_{j}^{-1} c_{k} \beta_{k}\right)=\sum_{j=1}^{n} c_{j} \beta_{j}
$$

for all $\beta_{j} \in R$ and any $1 \leq j \leq n$ in accordance with the conditions imposed on $c_{j}$ at the beginning of this section and $i N_{j}=N_{j} i$ for each $j$, the Fourier transform with the generator $i$ can be accomplished subsequently by each variable using Identity (19). The transform $x_{j} \mapsto\left(c_{j}\right)^{1 / 2} x_{j}$ is diagonal and $\left|\left(\cdots\left(c_{1}^{1 / 2} c_{2}^{1 / 2}\right) \cdots\right) c_{n}^{1 / 2}\right|=1$, so we can put $|D|=1$. Each Cayley-Dickson number can be presented in the polar form $z=|z| e^{\phi M}, \phi \in R, \quad|\phi| \leq \pi, M$ is a purely imaginary Cayley-Dickson number $|M|=1$, $\operatorname{Arg}(z)=(\phi+2 \pi k) M$ has the countable number of values, $k \in Z$ (see $\S 3$ in $[5,6])$. Therefore, we choose the branch $z^{1 / 2}=|z|^{1 / 2} \exp ((\operatorname{Arg} z) / 2),|z|^{1 / 2}>0$ for $z \neq 0$, with $|\operatorname{Arg}(z)| \leq \pi, \operatorname{Arg}(M)=M \pi / 2$ for each purely imaginary $M$ with $|M|=1$.

We treat the iterated integral as in $\S 6$, i.e. with the same order of brackets. Taking initially $c_{j} \in R$ and considering the complex analytic extension of formulas given above in each complex plane $R \oplus N_{j} R$ by $c_{j}$ for each $j$ by induction from 1 to $n$, when $c_{j}$ is not real in the operator $A, \operatorname{Im}\left(c_{j}\right) \in R N_{j}$, we get the fundamental solutions for $A$ with the form $(P(x)+c l 0)^{\lambda}$ instead of $(P(x)+c i 0)^{\lambda}$ with multipliers $\left(\cdots\left(c_{1}^{c / 2} c_{2}^{c / 2}\right) \cdots\right) c_{n}^{c / 2}$ instead of $\exp \left(\pi c\left(n-k_{+}\right) i / 2\right)$ as above and putting $|D|=1$. Thus

20) $\Psi\left(z_{1}, \cdots, z_{n}\right)=-\Gamma((n / 2)-1)\left(P^{*}\left(z_{1}, \cdots, z_{n}\right)-c l 0\right)^{1-(n / 2)}\left[\left(\cdots\left(c_{1}^{c / 2} c_{2}^{c / 2}\right) \cdots\right) c_{n}^{c / 2}\right]^{*} /\left(4 \pi^{n / 2}\right)$

for $3 \leq n$, while

21) $\Psi\left(z_{1}, z_{2}\right)=4^{-1}\left[c_{1}^{c / 2} c_{2}^{c / 2}\right]^{*} \operatorname{Ln}\left(P^{*}\left(z_{1}, z_{2}\right)-c l 0\right)$ for $n=2$,

since $c_{j}^{*}=c_{j}^{-1}$ for $\left|c_{j}\right|=1, y_{j} q_{j}=y_{j}\left(c_{j}^{c / 2}\right)^{*} q_{j} c_{j}^{1 / 2}$, while

$$
\left[\left(\cdots\left(\mathrm{d} c_{1}^{c / 2} q_{1} \mathrm{~d} c_{2}^{c / 2} q_{2}\right) \cdots\right) \mathrm{d} c_{n}^{c / 2} q_{n}\right]=\mathrm{d} q_{1} \cdots \mathrm{d} q_{n}\left[\left(\cdots\left(c_{1}^{c / 2} c_{2}^{c / 2}\right) \cdots\right) c_{n}^{c / 2}\right] \text { and }\left|\left(\cdots\left(c_{1}^{c / 2} c_{2}^{c / 2}\right) \cdots\right) c_{n}^{c / 2}\right|=1 \text {. }
$$

\subsection{Noncommutative Transforms of Products and Convolutions of Functions in the $A_{r}$ Spherical Coordinates}

For any Cayley-Dickson number $z=z_{0} i_{0}+\cdots+z_{2^{r}-1} i_{2^{r}-1}$ we consider projections
1) $\theta_{j}(z)=z_{j}, \quad z_{j} \in R$ or $C_{i}$ or $H_{J, K, L}$, $j=0, \cdots, 2^{r}-1, \theta_{j}(z)=\pi_{j}(z) i_{j}^{*}$,

given by Formulas $2(5,6)$ and $33(17)$. We define the following operators 
2) $R_{\alpha, j}\left(F^{n}(p ; \zeta)\right):=F^{n}\left(p_{0},(-1)^{\alpha_{1}} p_{1}, \cdots,(-1)^{\alpha_{j+1-\delta_{j, n}}} p_{j+1-\delta_{j, n}}\right.$,

$$
\left.p_{j+2-\delta_{j, n}}, \cdots p_{n} ; \zeta_{0},(-1)^{\alpha_{1}} \zeta_{1}+\pi \alpha_{1} / 2, \cdots,(-1)^{\alpha_{j+1-\delta_{j, n}}} \zeta_{j+1-\delta_{j, n}}+\pi \alpha_{j+1-\delta_{j, n}} / 2, \zeta_{j+2-\delta_{j, n}}, \cdots, \zeta_{n}\right)
$$

on images $F^{n}, 2^{r-1} \leq n \leq 2^{r}-1, j=0, \cdots, n$. For $\alpha_{j}$ and $\beta_{j} \in\{0,1\}$ their sum $\alpha_{j}+\beta_{j}$ is considered by $(\bmod 2)$, i.e. in the $\operatorname{ring} Z_{2}=Z /(2 Z)$, for two vectors $\alpha$ and $\beta \in\{0,1\}^{2^{r}-1}$ their sum is considered componentwise in $Z_{2}$. Let

3) $F^{n}(f ; u ; p ; \zeta)=\sum_{j=0}^{n} \sum_{k=0}^{2^{r}-1} \theta_{j}\left(F^{n}\left(\theta_{k}(f) ; u ; p ; \zeta\right)\right) i_{k} i_{j}$, also

$$
F_{j}^{n}(p ; \zeta):=\sum_{k=0}^{2^{r}-1} \theta_{j}\left(F^{n}\left(\theta_{k}(f) ; u ; p ; \zeta\right)\right) i_{k}
$$

for an original $f$, where $u(p, t ; \zeta)$ is given by Formulas $2(1,2,2.1)$. If $f$ is real or $C_{i}$ or $H_{J, K, L}$-valued, then $F_{j}^{n}=\theta_{j}\left(F^{n}\right)$.

Theorem. If $f$ and $g$ are two originals, then

4) $F^{n}(f g ; u ; p ; \zeta)=\sum_{j=0}^{n} \sum_{\alpha, \beta \in\{0,1\}^{n}}(-1)^{\alpha_{j+1}\left(1-\delta_{j+1, n}\right)}\left(R_{\alpha, j}\left(F_{j}^{n}\left(p-q_{0} ; \zeta-\eta\right)\right) * R_{\beta, j}\left(G_{j}^{n}\left(p+q_{0}-p_{0} ; \eta\right)\right) i_{j}\right.$,

4.1) $F^{n}\left(f^{*} g ; u ; p ; \zeta\right)=\sum_{j=0}^{n} \sum_{\alpha, \beta \in\{0,1\}^{n}}(-1)^{\alpha}{ }_{j+1}^{\left(1-\delta_{j+1, n}\right)}\left(R_{\alpha, j}\left(F_{j}^{n}(p ; \zeta-\eta)\right) R_{\beta, j}\left(G_{j}^{n}(p ; \eta)\right)\right) i_{j}$,

whenever $F^{n}(f g), F^{n}(f), F^{n}(g)$ exist, where $1 \leq n \leq 2^{r}-1,2 \leq r ; \quad \alpha_{k}+\beta_{k}=1(\bmod 2) \quad$ for $k \leq j$ or $k=j+1=n, \alpha_{k}+\beta_{k}=0(\bmod 2)$ for $k=j+1<n$ and $\alpha_{k}=\beta_{k}=0$ for $k>j+1$ in the $J$-th addendum on the right of Formulas $(4,4.1)$; the convolution is by $\left(p_{1}, \cdots, p_{n}\right)$ in (4), at the same time $q_{0} \in R$ and $\eta \in A_{r}$ are fixed.
Proof. The product of two originals can be written in the form:

5) $f(t) g(t)=\sum_{j=0}^{2^{r}-1} \sum_{k, l: i_{k} i_{l} i_{j}} \theta_{j}(f(t)) \theta_{l}(g(t)) i_{j}$.

The functions $\theta_{k}(f)$ and $\theta_{l}(g)$ are real or $C_{i}$ or $H_{J, K, L}$ valued respectively. The non-commutative transform of $f g$ is:

6) $F^{n}(f g)(p ; \zeta)=\int_{R^{n}} f(t) g(t) \exp (-u(p, t ; \zeta)) \mathrm{d} t=\left\{\int_{R^{n}}(f(t) g(t)) e^{-p_{0} s_{1}} \cos \left(p_{1} s_{1}+\zeta_{1}\right) i_{0} \mathrm{~d} t\right\}$

$$
\begin{aligned}
& +\left\{\sum_{j=2}^{n-1} \int_{R^{n}}(f(t) g(t)) e^{-p_{0} s_{1}} \sin \left(p_{1} s_{1}+\zeta_{1}\right) \cdots \sin \left(p_{j-1} s_{j-1}+\zeta_{j-1}\right) \cos \left(p_{j} s_{j}+\zeta_{j}\right) i_{j-1} \mathrm{~d} t\right\} \\
& +\int_{R^{n}}(f(t) g(t)) e^{-p_{0} s_{1}} \sin \left(p_{1} s_{1}+\zeta_{1}\right) \cdots \sin \left(p_{n} s_{n}+\zeta_{n}\right) i_{n} \mathrm{~d} t .
\end{aligned}
$$

On the other hand,

$$
\text { 7) } \int_{R^{n}} f(t) g(t) e^{-p_{0} s_{1}+i \sum_{j=1}^{k}\left(p_{j} s_{j}+\zeta_{j}\right) \gamma_{j}} \mathrm{~d} t=\int_{R^{n}}\left(\int_{R^{n}} f(t) e^{-\left(p_{0}-q_{0}\right) s_{1}+i \sum_{j=1}^{k}\left(\left(p_{j}-q_{j}\right) s_{j}+\zeta_{j}-\eta_{j}\right) \gamma_{j}} \mathrm{~d} t\right)\left(\int_{R^{n}} g(t) e^{-q_{0} s_{1}+i \sum_{j=1}^{k}\left(q_{j} s_{j}+\eta_{j}\right) \gamma_{j}} \mathrm{~d} t\right) \mathrm{d} q,
$$

where $k=1,2, \cdots, n, \quad \gamma_{j} \in\{-1,1\}$. Therefore, using Euler's formula $e^{i \varphi}=\cos (\phi)+i \sin (\phi)$ and the trigonometric formulas

$$
\begin{gathered}
\cos (\phi+\psi)=\cos (\phi) \cos (\psi)-\sin (\phi) \sin (\psi), \\
\sin (\phi+\psi)=\sin (\phi) \cos (\psi)+\cos (\phi) \sin (\psi)
\end{gathered}
$$

for all $\phi, \psi \in R$, and Formulas $(6,7)$, we deduce ex- pressions for $\theta_{j}\left(F^{n}(f g)\right)$. We get the integration by $q_{1}, \cdots, q_{n}$, which gives convolutions by the $p_{1}, \cdots, p_{n}$ variables. Here $q_{0} \in R$ and $\eta \in A_{r}$ are any marked numbers. Thus from Formulas $(5-7)$ and $2(1,2,2.1,4)$ we deduce Formula (4).

Moreover, one certainly has

8) $\int_{R^{n}}\left(f^{*} g\right)(t) e^{-p_{0} s_{1}+i \sum_{j=1}^{k}\left(p_{j^{\prime}}+\zeta_{j}\right) \gamma_{j}} \mathrm{~d} t=\left(\int_{R^{n}} f(t) e^{-p_{0} s_{1}+i \sum_{j=1}^{k}\left(p_{j} s_{j}+\zeta_{j}-\eta_{j}\right) \gamma_{j}} \mathrm{~d} t\right)\left(\int_{R^{n}} g(t) e^{-p_{0} s_{1}+i \sum_{j=1}^{k}\left(p_{j} s_{j}+\eta_{j}\right) \gamma_{j}} \mathrm{~d} t\right)$ 
for each $1 \leq k \leq n, \gamma_{j} \in\{-1,1\}$, since $s_{j}(t)=s_{j}(t-\tau)+s_{j}(\tau)$ for all $j=1, \cdots, n$ and $t, \tau \in R^{n}$. Thus from Relations $(6,8)$ and $2(1,2,2.1,4$ and Euler's formula one deduces expressions for $\theta_{j}\left(F^{n}\left(f^{*} g\right)\right)$ and Formula (4.1).

\subsection{Moving Boundary Problem}

Let us consider a boundary problem

1) $A f=g$ in the half-space $t_{n} \geq \phi\left(t_{n}\right)$, where $\phi(0)=0$ and $\phi\left(t_{n}\right)<t_{n}$ for each $0 \leq t_{n} \in R$. Suppose that the function $t_{n}-\phi\left(t_{n}\right)=: \psi\left(t_{n}\right)$ is differentiable and bijective. For example, if $0<v<1$ and $\phi\left(t_{n}\right)=v t_{n}$, then the boundary is moving with the speed $v$. Make the change of variables

$$
y_{n}=\psi\left(t_{n}\right), y_{1}=t_{1}, \cdots, y_{n-1}=t_{n-1},
$$

then

$$
t_{n}=\psi^{-1}\left(y_{n}\right) \text { and } \mathrm{d} t_{n}=\mathrm{d} s_{n}=\left(\mathrm{d} t_{n} / \mathrm{d} y_{n}\right) \mathrm{d} y_{n}
$$

and due to Theorem 25 we infer that

2) $F^{n}\left(\sum_{|\alpha| \leq m} b_{\alpha} \partial_{s}^{\alpha} \chi_{y_{n} \geq 0} f(t) ; p ; \zeta\right)=\sum_{|\alpha| \leq m, 0 \leq q_{n} \leq \alpha_{n}-1} b_{\alpha}\left(\delta_{0, \alpha_{n}}-1\right)$

$$
\begin{aligned}
& \left(p_{0}+S_{e_{1}} p_{1}\right)^{\alpha_{1}} p_{2}^{\alpha_{2}} \cdots p_{n-1}^{\alpha_{n-1}} p_{n}^{\alpha_{n}-q_{n}-1} S_{\alpha-\alpha_{1} e_{1}-\left(q_{n}+1\right) e_{n}} F^{n-1, y^{n}}\left(\partial_{t_{n}}^{q_{n}} w(y), u\left(p,\left(y^{n}\right) ; \zeta\right) ; p ; \zeta\right) \\
& +\sum_{|\alpha| \leq m} b_{\alpha}\left(p_{0}+S_{e_{1}} p_{1}\right)^{\alpha_{1}} p_{2}^{\alpha_{2}} \cdots p_{n}^{\alpha_{n}} S_{\alpha-\alpha_{1} e_{1}} F^{n}\left(\chi_{y_{n} \geq 0}(y) w(y) ; p ; \zeta\right)=G^{n}(p ; \zeta)
\end{aligned}
$$

in the $A_{r}$ spherical coordinates and

$$
\text { 2.1) } \begin{aligned}
& F^{n}\left(\sum_{|\alpha| \leq m} a_{\alpha} \partial_{t}^{\alpha} \chi_{y_{n} \geq 0} f(t) ; p ; \zeta\right)=\sum_{|\alpha| \leq m, 0 \leq q_{n} \leq \alpha_{n}-1} a_{\alpha}\left(\delta_{0, \alpha_{n}}-1\right) \\
& \left(p_{0}+S_{e_{1}} p_{1}\right)^{\alpha_{1}}\left(p_{0}+p_{2} S_{e_{2}}\right)^{\alpha_{2}} \cdots\left(p_{0}+p_{n-1} S_{e_{n-1}}\right)^{\alpha_{n-1}}\left(p_{0}+p_{n} S_{e_{n}}\right)^{\alpha_{n}-q_{n}-1} F^{n-1, y^{n}}\left(\partial_{t_{n}}^{q_{n}} w(y), u\left(p,\left(y^{n}\right) ; \zeta\right) ; p ; \zeta\right) \\
& +\sum_{|\alpha| \leq m} a_{\alpha}\left(p_{0}+S_{e_{1}} p_{1}\right)^{\alpha_{1}}\left(p_{0}+p_{2} S_{e_{2}}\right)^{\alpha_{2}} \cdots\left(p_{0}+p_{n} S_{e_{n}}\right)^{\alpha_{n}} F^{n}\left(\chi_{y_{n} \geq 0}(y) w(y) ; p ; \zeta\right)=G^{n}(p ; \zeta)
\end{aligned}
$$

in the $A_{r}$ Cartesian coordinates, where $w(y):=f(t(y))\left(\mathrm{d} t_{n} / \mathrm{d} y_{n}\right)$.

Expressing $F^{n}\left(\chi_{y_{n} \geq 0}(y) w(y) ; p ; \zeta\right)$ through $G^{n}(p ; \zeta)$ and the boundary terms

$$
F^{n-1, y^{n}}\left(\partial_{t_{n}}^{q_{n}} w(y), u\left(p,\left(y^{n}\right)\right) ; \zeta ; p ; \zeta\right)
$$

as in $\S 28.3$ and making the inverse transform 8(4) or $8.1(1)$, or using the integral kernel $\xi$ as in $\S 28.5$, one gets a solution $w(y)$ or

$$
f(t)=w(y(t))\left(\mathrm{d} y_{n}\left(t_{n}\right) / \mathrm{d} t_{n}\right) \quad \text { See reference [21-30]). }
$$

\subsection{Partial Differential Equations with Discontinuous Coefficients}

Consider a domain $U$ and its subdomains

$U \supset U_{1} \supset \cdots \supset U_{k}$ satisfying Conditions $28(D 1, D 4$, i-vii) so that coefficients of an operator $A$ (see 28(2)) are constant on $\operatorname{Int}\left(U_{k}\right)$ and on $V_{1}=U \backslash \operatorname{Int}\left(U_{1}\right)$, $V_{2}=U_{1} \backslash \operatorname{Int}\left(U_{2}\right), \cdots, V_{k}=U_{k-1} \backslash \operatorname{Int}\left(U_{k}\right)$ and are allowed to be discontinuous at the common borders $\partial V_{j} \cap \partial U_{j}$ for each $j=1, \cdots, k$. Each function $f \chi_{U_{j}}$ is an original on $U$ or a generalized function with the support $\operatorname{supp}\left(f \chi_{U_{j}}\right) \subset U_{j}$ if $f$ is an original or a generalized function on $U$. Choose operators $A^{j}$ with constant coefficients on $U^{j}$ and $\left.A^{j}\right|_{\operatorname{Int}\left(V_{j}\right)}=0$, where $U^{0}=U$, so that $\left.A\right|_{U_{k}}=A^{k}, \cdots,\left.A\right|_{U_{j}}=A^{j^{j}}+\cdots+A^{k}, \cdots$,
$\left.A\right|_{U}=A^{0}+\cdots+A^{k}$. Therefore, in the class of originals or generalized functions on $U$ the problem (see 28(1,2)) can be written as

1) $A f=g$, or

2) $A^{0} f \chi_{V_{1}}=g \chi_{V_{1}}, \cdots, A^{k-1} f \chi_{V_{k}}=g \chi_{V_{k}}$,

$$
A^{k} f \chi_{U_{k}}=g \chi_{U_{k}},
$$

since $\chi_{V_{1}}+\cdots+\chi_{V_{k}}+\chi_{U_{k}}=\chi_{U}$. Thus the equivalent problem is:

3) $A^{0} f^{0}=g^{0}, A^{1} f^{1}=g^{1}, \cdots, A^{k} f^{k}=g^{k}$

with $f^{k}=f \chi_{U_{k}}, g^{k}=g \chi_{U_{k}}$, also $f^{j}=f \chi_{V_{j+1}}$,

$g^{j}=g \chi_{V_{j+1}}$ for each $j=0, \cdots, k-1$. On $\partial U$ take the boundary condition in accordance with 28(5.1). With any boundary conditions in the class of originals or generalized functions on additional borders $\partial U_{j} \backslash \partial U$ given in accordance with 28(5.1) a solution $f^{j}$ on $U^{j}$ exists, when the corresponding condition 8(3) is satisfied (see Theorems 8 and 28.1).

Each problem $A^{j} f^{j}=g^{j}$ can be considered on $U_{j}$, since $\operatorname{supp}\left(g^{j}\right) \subset U_{j}$. Extend $f^{j}$ by zero on $U \backslash V_{j}$ for each $0 \leq j \leq k-1$. When the right side of 28(6) is non-trivial, then $f^{j}$ is non-trivial. If $f^{j-1}$ is calculated, then the boundary conditions on $\partial U^{j} \backslash \partial U$ can be chosen in accordance with values of $f^{j-1}$ and its 
corresponding derivatives $\left.\left(\partial^{\beta} f^{j-1} / \partial v^{\beta}\right)\right|_{\left(\partial U^{j} \backslash \partial U\right)}$ for some $\beta<\operatorname{ord}\left(A^{j}\right)$ in accordance with the operator $A^{j}$ and the boundary conditions $28(5.1)$ on the boundary $\partial U^{j} \backslash \partial U$. Having found $f^{j}$ for each $j=0, \cdots, k$ one gets the solution $f=f^{0}+\cdots+f^{k}$ on $U$ of Problem (1) with the boundary conditions $28(5.1)$ on $\partial U$.

\section{REFERENCES}

[1] V. S. Vladimirov, "Equations of Mathematical Physics," Nauka, Moscow, 1971.

[2] M. A. Lavretjev and B. V. Shabat, "Methods of Functions of the Complex Variable," Nauka, Moscow, 1987.

[3] B. van der Pol and H. Bremmer, "Operational Calculus Based on the Two-Sided Laplace Integral," Cambridge University Press, Cambridge, 1964.

[4] S. V. Ludkovsky, "The Two-Sided Laplace Transformation over the Cayley-Dickson Algebras and Its Applications," Journal of Mathematical Sciences, Vol. 151, No. 5, 2008, pp. 3372-3430. doi:10.1007/s10958-008-9038-y

[5] S.V. Lüdkovsky and F. van Oystaeyen, "Differentiable Functions of Quaternion Variables," Bulletin des Sciences Mathématiques, Vol. 127, No. 9, 2003, pp. 755-796. doi:10.1016/S0007-4497(03)00063-0

[6] S. V. Ludkovsky, "Differentiable Functions of CayleyDickson Numbers and Line Integration," Journal of Mathematical Sciences, Vol. 141, No. 3, 2007, pp. 1231-1298. doi:10.1007/s10958-007-0042-4

[7] W. R. Hamilton, "Selected Works. Optics. Dynamics. Quaternions," Nauka, Moscow, 1994.

[8] J. C. Baez, "The Octonions," Bulletin of the American Mathematical Society, Vol. 39, No. 2, 2002, pp. 145-205. doi:10.1090/S0273-0979-01-00934-X

[9] I. L. Kantor and A. S. Solodovnikov, "Hypercomplex Numbers," Springer-Verlag, Berlin, 1989. doi:10.1007/978-1-4612-3650-4

[10] A. G. Kurosh, "Letures on General Algebra," Nauka, Moscow, 1973.

[11] H. Rothe, "Systeme Geometrischer Analyse," In: Encyklopädie der Mathematischen Wissenschaften, Geometrie, Teubner, Leipzig, Vol. 3, 1914-1931, pp. 1277-1423.

[12] G. Emch, "Méchanique Quantique Quaternionienne et Relativit'èrestreinte," Helvetica Physica Acta, Vol. 36, 1963, pp. 739-788.

[13] F. Gürsey and C.-H. Tze, "On the Role of Division, Jordan and Related Algebras in Particle Physics," World Scientific Publishing Co., Singapore, 1996.

[14] H. B. Lawson and M.-L. Michelson, "Spin Geometry," Princeton University Press, Princeton, 1989.
[15] M. A. Solovjev, "A Structure of a Space of Non-Abelian Gauge Fields," Proceeding of Lebedev Physical Institute, No. 210, 1993, pp. 112-155.

[16] S. V. Ludkovsky, "Differential Equations over Octonions," Advances in Applied Clifford Algebras, Vol. 21, No. 4, 2011, pp. 773-797. doi:10.1007/s00006-011-0286-4

[17] E. H. Spanier, “Algebraic Topology," Academic Press, New York, 1966.

[18] L. I. Kamynin, "Course of Mathematical Analysis," Moscow State University Press, Moscow, 1993.

[19] V. A. Zorich, "Mathematical Analysis," Nauka, Moscow, Vol. 2, 1984.

[20] I. Rubinstein and L. Rubinstein, "Partial Differential Equations in Classical Mathematical Physics," Cambridge University Press, Cambridge, 1998.

[21] I. M. Gelfand and G. E. Shilov, "Generalized Functions and Operations with Them," Fiziko-Mathematicheskaya Literatura, Moscow, 1958.

[22] S. V. Ludkovsky, "Feynman Integration over Octonions with Application to Quantum Mechanics," Mathematical Methods in the Applied Sciences, Vol. 33, No. 9, 2010, pp. 1148-1173.

[23] S. V. Ludkovsky and W. Sproessig, "Ordered Representations of Normal and Super-Differential Operators in Quaternion and Octonion Hilbert Spaces," Advances in Applied Clifford Algebras, Vol. 20, No. 2, 2010, pp. 321342. doi:10.1007/s00006-009-0180-5

[24] S. V. Ludkovsky, "Algebras of Operators in Banach Spaces over the Quaternion Skew Field and the Octonion Algebra," Journal of Mathematical Sciences, Vol. 144, No. 4, 2008, pp. 4301-4366. doi:10.1007/s10958-007-0273-4

[25] W. Arendt, C. J. K. Batty, M. Hieber and F. Neubrander, "Vector-Valued Laplace Transforms and Cauchy Problems,” Birkhäüuser, Basel, 2001.

[26] L. Berg, "Einfürung in Die Operatorenrechnung," VEB Deutscher Verlag der Wissenschaften, Berlin, 1965.

[27] U. Graf, “Applied Laplace Transform for Scientists and Engineers," Birkhäuser, Basel, 2004. doi:10.1007/978-3-0348-7846-3

[28] J. Leray, "Un Prologement de la Transformation de Laplace qui Transforme la Solution Unitaire d'un op e'rateur Hyperbolique en sa Solution e'Lèmentaire," Bulletin de la Société Mathématique de France, Vol. 90, 1962, pp. 39-156.

[29] S. V. Ludkovsky, "Functions of Several Cayley-Dickson Variables and Manifolds over Them," Journal of Mathematical Sciences, Vol. 141, No. 3, 2007, pp. 1299-1330. doi:10.1007/s10958-007-0043-3

[30] A. P. Prudnikov, Yu. A. Brychkov and O. I. Marichev, "Integrals and Series," Nauka, Moscow, 1981. 